\title{
25 Research Soure \\ Stability and Sensitivity Analysis and Optimization Control of the Hydro-turbine Generator Unit
}

\section{Yousong Shi ( $\nabla 1432317459 @ q q . c o m)$}

Huazhong University of Science and Technology - Main Campus: Huazhong University of Science and Technology

Jianzhong Zhou

Huazhong University of Science and Technology - Main Campus: Huazhong University of Science and Technology

\section{Research Article}

Keywords: Sensitivity, Stability, Hydro-turbine governing system, Shafting system, Multi-objective optimization control, Nonlinear dynamic

Posted Date: April 16th, 2021

DOl: https://doi.org/10.21203/rs.3.rs-348512/v1

License: (c) (i) This work is licensed under a Creative Commons Attribution 4.0 International License.

Read Full License 
Stability and sensitivity analysis and multi-objective optimization control of the hydro-turbine generator unit Yousong Shi Jianzhong Zhou* Jiapeng Ren Baonan Liu Yuxin Li Wei Liu (School of civil and hydraulic engineering, Hua Zhong university of science and technology, Wuhan 430074, China)

\section{Abstract}

The hydro-turbine governing system (HTGS) and shafting system are mutually coupled. However, the interaction between them has always been neglected. This paper aims to explore the stability and sensitivity of the governor control parameters to the HTGS and shafting system and make the optimal control of the stable operation for the hydro-turbine generator unit(HTGU). First, a novel HTGU motion equation is proposed, which can make connections between the HTGS and the shafting system of the HTGU. And on this basis, the nonlinear coupling mathematical model of the HTGS and the shafting system is established. According to the nonlinear mathematical model, the sensitivity of the governor control parameters on the operating stability of the HTGU is obtained. Then, a multi-objective governor control parameters optimization strategy is proposed. Furthermore, the chaotic-dominated sorting genetic algorithm II(NSGA-II) and multi-objective evolutionary algorithm based on decomposition(MOEAD) were introduced to obtain the optimal control parameter and mutually verify the effectiveness of the optimization effect. Finally, the nonlinear dynamic characteristics of HTGU under optimal control were revealed. The simulation results show that the rotation speed deviation and shafting system vibrations are sensitive on the PID parameters in some ranges and the stable region will be decreased when considering the shafting system vibrations. The multi-objective PID parameter optimization strategy shows good control performance on the nonlinear dynamic characteristics of the HTGU. The shafting system vibrations excited by the coupled vibration sources are quasi-period in 3D space. In addition to this, these results and the optimization strategy can provide some bases for the design and stable operation of the HTGU. 


\section{keywords}

Sensitivity, Stability, Hydro-turbine governing system, Shafting system, Multi-objective optimization control, Nonlinear dynamic.

\section{List of symbols}

\begin{tabular}{|c|c|}
\hline Symbol & Symbolic meaning \\
\hline$h$ & Hydro-turbine working head $(H)$ \\
\hline$q_{t}$ & Flows of the penstock $(Q)$ \\
\hline$h_{t 0}$ & Head loss of penstock \\
\hline$T_{w t}$ & Penstock flows inertia time constant \\
\hline$x$ & The HTGU speed $(n)$ relative deviation \\
\hline$y$ & Guide vane relative opening $(Y)$ \\
\hline$m_{t}$ & The relative dynamic torque $\left(M_{t}\right)$ \\
\hline$m_{g}$ & The relative resistance torque $\left(M_{g}\right)$ \\
\hline$e_{h}, e_{x}, e_{y}$ & Hydro-turbine torque transfer coefficient \\
\hline$e_{q x}, e_{q y}, e_{q h}$ & Hydro-turbine flow transfer coefficient \\
\hline$T_{a b}$ & The inertia time constant of the HTGU \\
\hline$k_{p}$ & The proportional gain \\
\hline$k_{i}$ & The integral gain \\
\hline$k_{d}$ & The differential gain \\
\hline$T_{y}$ & Main servomotor time constant \\
\hline$u$ & Governor regulating output \\
\hline$e_{g}$ & Load self-regulation coefficient \\
\hline$a$ & Center distance between upper guide bearing and generator \\
\hline$b$ & Center distance between lower guide bearing and generator \\
\hline$c$ & Center distance between lower guide bearing and hydro-turbine guide bearing \\
\hline$d$ & Distance between hydro-turbine guide bearing and center of the hydro-turbine runner \\
\hline$l$ & Length of spindle \\
\hline$L$ & Generator rotor height \\
\hline$D$ & Generator rotor diameter \\
\hline$B$ & The magnetic density \\
\hline$\beta$ & General coefficient \\
\hline$e_{f}$ & The dynamic eccentricity distance of the generator \\
\hline$\delta_{1}$ & The normal clearance of generator rotor and stator \\
\hline$K$ & The equivalent stiffness of the sealing forces \\
\hline$D_{s}$ & The equivalent damping coefficient \\
\hline$\tau_{f}$ & The nonlinear function of displacement perturbation \\
\hline$e_{t}$ & The relative eccentricity of the hydro-turbine runner \\
\hline$\delta_{2}$ & The sealing clearance of hydro-turbine runner \\
\hline$K_{z}$ & The undetermined coefficient of axial hydrodynamic thrust \\
\hline$D_{1}$ & The hydro-turbine runner diameter \\
\hline$m_{11}$ & The equivalent mass of the generator spindle and generator \\
\hline$m_{22}$ & The equivalent mass of the hydro-turbine spindle and hydro-turbine \\
\hline$g$ & The gravitational acceleration \\
\hline$r_{1}$ & Radial displacement of the generator \\
\hline
\end{tabular}




\begin{tabular}{|c|c|}
\hline$r_{2}$ & Radial displacement of the hydro-turbine runner \\
\hline$r_{3}$ & Radial displacement of the upper guide bearing \\
\hline$r_{4}$ & Radial displacement of the lower guide bearing \\
\hline$r_{5}$ & Radial displacement of the hydro-turbine guide bearing \\
\hline$m_{2}$ & The mass of the hydro-turbine runner \\
\hline$m_{1}$ & The mass of the generator rotor \\
\hline$e_{1}$ & The generator mass eccentricity distance \\
\hline$e_{2}$ & The hydro-turbine runner mass eccentricity distance \\
\hline$j_{1}$ & Rotary inertia of the generator \\
\hline$j_{2}$ & Rotary inertia of the hydro-turbine runner \\
\hline$\phi_{1}$ & Phase position of the generator \\
\hline$\phi_{2}$ & Phase position of the hydro-turbine runner \\
\hline$\left(x_{1}, y_{1}, z_{1}\right)$ & The central coordinates of the generator \\
\hline$\left(x_{2}, y_{2}, z_{2}\right)$ & The central coordinates of the hydro-turbine runner \\
\hline$K_{1}$ & The stiffness of the upper guide bearing \\
\hline$K_{2}$ & The stiffness of the lower guide bearing \\
\hline$K_{3}$ & The stiffness of the hydro-turbine guide bearing \\
\hline$K_{y}$ & The elastic coefficient of the spindle \\
\hline$k_{z}$ & The thrust guide bearing stiffness \\
\hline$k_{l}$ & The axial stiffness \\
\hline$d_{H}$ & The inner diameter of the spindle \\
\hline$d_{B}$ & The external diameter of the spindle \\
\hline$l$ & The total length of the generator lower spindle and the hydro-turbine spindle \\
\hline$E$ & Elastic modulus of the spindle \\
\hline$c_{1}$ & The damping coefficient of the generator \\
\hline$c_{2}$ & The damping coefficient of the hydro-turbine runner \\
\hline$c_{t}$ & The torsional damping coefficient of the spindle \\
\hline$F_{x i}$ & The excitation force in the horizontal $x$ direction \\
\hline$F_{y i}$ & The excitation force in the horizontal $y$ direction \\
\hline$F_{z i}$ & The excitation force in the horizontal $z$ direction \\
\hline$T$ & The total kinetic energy of the HTGU \\
\hline$U$ & The elastic potential energy of the HTGU \\
\hline$Q_{i}$ & The corresponding generalized force \\
\hline$M_{g}$ & The generator magnetic torque \\
\hline$M_{t}$ & The hydro-turbine torque \\
\hline$\varphi_{1}$ & The initial phase of the generator \\
\hline$\varphi_{2}$ & The initial phase of the hydro-turbine runner \\
\hline$\alpha$ & Torsion angle of the spindle \\
\hline$\omega$ & The angular speed of the HTGU \\
\hline PID-MOO & PID parameters multi-objective optimized \\
\hline PID-Stein & PID parameters obtained by Stein's formula \\
\hline
\end{tabular}

\section{Introduction}


are conventional hydropower generation, pumped storage power generation, tidal power generation, ocean current

power generation. They mainly play the role of peak and frequency regulation in the power grid, especially conventional hydropower generation and pumped storage power generation [2-3]. Therefore, the efficient, safe, and stable operation of the HTGU has always been the hotspot and difficulty in the research, which mainly involves the stability, the opening and stopping strategy, the equipment fault diagnosis, and the status evaluation, etc. Of course, stability is the most important one that is concerned with the safety and stability of the power grid and the HTGU.

In recent years, the research on the stability of HTGU has been divided into two main directions: the coupling shafting system of the hydro-turbine governing system and the coupling power network of the hydro-turbine governing system [4-5]. They are all complex nonlinear systems, and the study of their complex nonlinear dynamic behavior is of great significance for the realization of optimal economic operation control [6]. In previous works, the stability and dynamic characteristics of the HTGS were studied using the Routh-Hurwitz criterion [7], root locus method [8], state-space method [9], and Hopf bifurcation theory [10-12]. In [13], Hopf bifurcation theory is applied to analyze the influence of the structural parameters of the sloping ceiling tailrace tunnel on the stability domain of the PID control parameters. In $[11,14]$, the simulation results show that the delay and saturation of the servo-system have a certain degree of impact on the stability of the HTGS. At present, in the practical application of engineering, PID is the most important controller. Some scholars have also proposed fractional order PID controller, sliding mode controller, state feedback controller, and so on. $\operatorname{In}[15]$, a novel mathematical model of Francis HTGS with a straight-tube surge tank is proposed, where the unstable oscillations of this model are studied extensively and presented in the forms of the bifurcation diagram, time waveform plot, phase trajectories, and power spectrum. To eliminate these undesirable behaviors, a specified fuzzy sliding mode controller is designed. In[16], the state equation of HTGS with the super long headrace tunnel(SLHT) was derived, and the robust $\mathrm{H} \infty$ control strategy is designed based on the exact linearization of HTGS with SLHT and the construction of 
nominal output function (NOF). In[17], it was focused on the design of the fractional-order PID (FOPID) controller for

the HTGS and the optimal control parameters were obtained using NSGA-II. In[18], the fuzzy fractional-order PID (FFOPID) controller is designed to improve the stability of the nonlinear pumped storage unit(PSU), and the control parameters of the FOPID are optimized based on the multi-objective gravitational search algorithm (MOGSA).

As far as shafting system vibration is concerned, the research on vibration characteristics gradually develops from single vibration under a single excitation to multi-directional vibration under the influence of the coupled vibration sources. Such as the differential equation of lateral vibration of the shafting is established, and the bifurcated characteristics of the vibration caused by rotor mass eccentricity and rotor speed of the HTGU are revealed [19, 20]. The torsional vibration characteristics of the HTGU are analyzed, meanwhile, the influences of the UMP [21], the fluid seal excitation [22], the rotors Rub-impact [23], the hydraulic unbalance [24], the guide bearing loose [25], the gyroscopic effect [26], the shaft misalignment [27], and pressure pulsation of the draft tube [28] effect on shafting system vibrations are investigated. With the further development of the research, the hydraulic-mechanical-electrical coupling factors are considered in the shafting system to explore the nonlinear vibration dynamic behavior. A mathematical model considering the coupling relationship between hydraulic system, electrical system, and guide vane mechanical system was established, the sensitivity of various parameters was revealed [29-31]. In [32], the generalized Hamiltonian model of the shafting system is established which provides a new idea for exploring shafting vibration. In [33], the fractional-order dynamic model of the shafting system and the HTGS is established to investigate the shafting vibration nonlinear dynamic behavior. Besides, The 3D FEA model for the HTGU coupling foundation of hydropower station is established by taking the guide bearing as the bridge connecting the shafting and the structure of the hydropower house, and then the influence of the shafting system vibration on hydropower house vibration and its response mechanism is studied numerically $[34,35]$.

To sum up, the stability and dynamic characteristics of HTGS are obtained by different methods, and some controllers are proposed to achieve effective control. On the other hand, the shafting system vibrations dynamic 
characteristics under different excitation sources are verified, which lays a theoretical foundation for vibration control and fault diagnosis of the HTGU. However, the stability and sensitivity of the PID parameters to the HTGS and the shafting system have not been revealed. The nonlinear dynamic characteristics of the HTGS and shafting system vibration under the coupling effects of the mass eccentricity, the UMP, the arcuate whirl of the rotor, and the seal excitation under the optimal PID control haven't been explored which seriously restricted the secure and stable operation of the HTGU, and brought huge economic losses. Therefore, it is urgent to explore the stability and sensitivity of the PID parameter under the action of hydraulic-mechanical-electrical coupling vibration sources and realize optimal control to ensure the safe and stable operation of the HTGU.

As summarized above, the novelty and contributions of this study are: (1) A novel and practical nonlinear mathematical model coupling HTGS and shafting system is established. (2) The stability and sensitivity of ration speed deviation and shafting system vibrations to the PID parameters are revealed. (3) The parameters chosen of the PID governor are formulated as a multi-objective optimization problem that considers the HTGS and shafting system vibrations simultaneously for the first time, in which the objective functions are composed by the ITSE. (4)The influence of the self-regulating coefficient $\left(e_{g}\right)$ on the nonlinear dynamic characteristics of the HTGU is revealed. (5) The nonlinear dynamic characteristics of the shafting system vibrations in the 3D space, which are generated by the mass eccentricity, the UMP, the arcuate whirl of the rotor, and the seal excitation coupled vibration sources under the optimal control are revealed. Of course, there are still some shortcomings in this paper, the gyroscopic effect for shafting vibration is ignored. Meanwhile, the idealized models are adopted for external excitation sources of hydraulic, mechanical, and electrical systems, which cannot fully reflect the external excitation action on the HTGU.

The remainder of this paper is organized as follows. In section 2, the nonlinear mathematical modeling. In section 3 , the stability and sensitivity of the governor PID parameters to the HTGU. In section 4, the governor PID parameter multi-objective parameter optimization control. In section 5, the nonlinear dynamic characteristics of the HTGU. Finally, in section 6 , the conclusions are presented. 


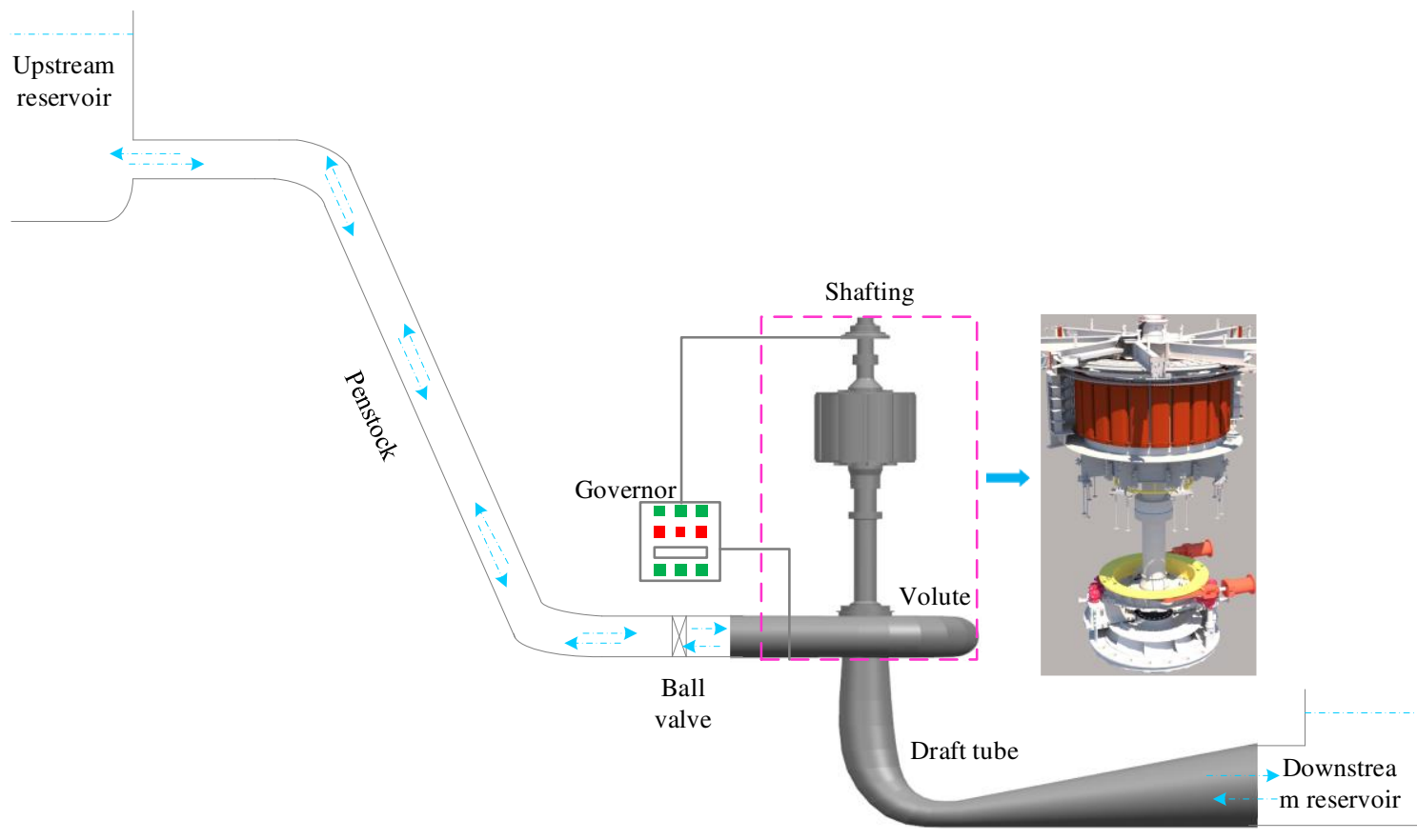

2.1 Hydro-turbine governing system equation model of the HTGS is established as follows:

(1) Dynamic equation of linear penstock [13]:

$$
h=-T_{w t} \frac{\mathrm{d} q_{t}}{\mathrm{~d} t}-\frac{2 h_{t 0}}{H_{0}} q_{t}
$$

in Eq (1), $h=\left(H-H_{0}\right) / H_{0}, q_{t}=\left(Q-Q_{0}\right) / Q_{0}$ are the working head $(H)$, and the flows of the penstock $(Q)$ of the HTGU, respectively. The subscript " 0 " represents the initial value, $h_{t 0}$ is the head loss of penstock, $T_{w t}$ is the inertia time constant of flows in the penstock. 
(2) Hydro-turbine torque and flows equation [13]:

$$
\begin{aligned}
& m_{t}=e_{h} h+e_{x} x+e_{y} y \\
& q_{t}=e_{q h} h+e_{q x} x+e_{q y} y
\end{aligned}
$$

2 (3) Generator motion equation [13]:

$$
\frac{\mathrm{d} x}{\mathrm{~d} t}=\frac{1}{T_{a b}}\left(m_{t}-m_{g}-e_{g} x\right)
$$

3 (4) Electro-hydraulic servo system differential equation [36]:

$$
\frac{\mathrm{d} y}{\mathrm{~d} t}=\frac{u-y}{T_{y}}
$$

4 (5) PID controller equation[36]:

$$
\frac{\mathrm{d} u}{\mathrm{~d} t}=-k_{p} \frac{\mathrm{d} x}{\mathrm{~d} t}-k_{i} x-k_{d} \frac{\mathrm{d}^{2} x}{\mathrm{~d} t^{2}}
$$

$$
\begin{aligned}
& \mathscr{\phi}_{t}=\left(-\frac{q_{t}-e_{q x} x-e_{q y} y}{e_{q h}}-\frac{2 h_{t 0}}{H_{0}} q_{t}\right) / T_{w t} \\
& \&=\frac{1}{T_{a b}}\left(\frac{e_{h} q_{t}}{e_{q h}}+\left(e_{x}-e_{g}-\frac{e_{h} e_{q x}}{e_{q h}}\right) x+\left(e_{y}-\frac{e_{h} e_{q y}}{e_{q h}}\right) y-m_{g}\right) \\
& \&=\frac{u-y}{T_{y}} \\
& \iota \&=\frac{-k_{p}}{T_{a b}}\left(e_{h} \frac{q_{t}-e_{q x} x-e_{q y} y}{e_{q h}}+e_{x} x+e_{y} y-m_{g}-e_{g} x\right)-k_{i} x-\frac{k_{d}}{T_{a b}}\left(\frac{e_{h}}{e_{q h}} \&+\left(e_{x}-e_{g}-\frac{e_{h} e_{q x}}{e_{q h}}\right) \&+\left(e_{y}-\frac{e_{h} e_{q y}}{e_{q h}}\right) \&\right)
\end{aligned}
$$



hydro-turbine runner, and upper, lower, and hydro-turbine guide bearings, respectively. We have $r_{i}=\sqrt{\left(x_{i}^{2}+y_{i}^{2}\right)}$, and we denote $\left|O_{1} O_{3}\right|=a,\left|O_{1} O_{4}\right|=b,\left|O_{4} O_{5}\right|=c,\left|O_{2} O_{5}\right|=d$. From the geometrical relation in Fig. 2, the following equations can be obtained [34]:

$$
\begin{gathered}
r_{3}=\frac{[(a+b)(b+c+d)-a(c+d)] r_{1}+a b r_{2}}{b(b+c+d)} \\
r_{4}=\frac{(c+d) r_{1}+b r_{2}}{b+c+d} \\
r_{5}=\frac{d r_{1}+(b+c) r_{2}}{b+c+d}
\end{gathered}
$$

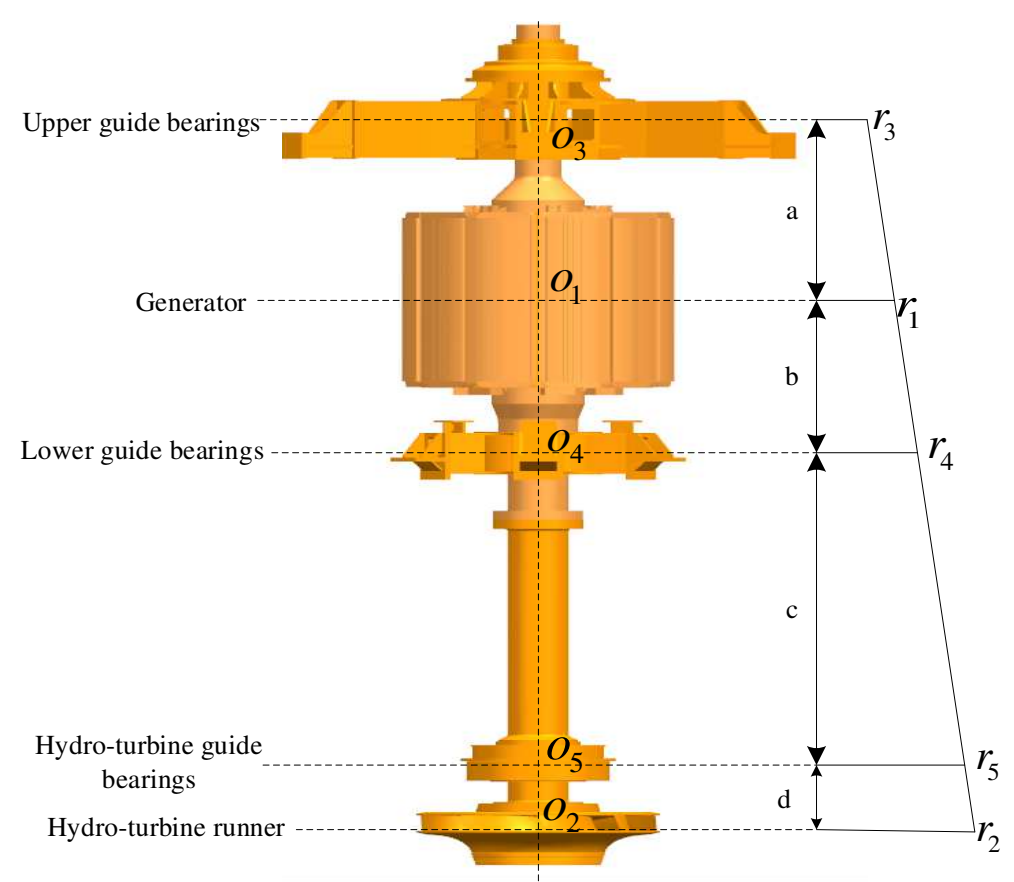

Fig. 2 Shafting structure diagram 
are mainly distributed on the generator and the hydro-turbine runner, the calculation formula can be expressed as[23]:

$$
F=m e \omega^{2}
$$

The electrical vibration source is the UMP, which is calculated as follows [19]:

$$
F_{u m p}=\beta \pi D L\left(\frac{B}{0.5}\right)^{2} \frac{e_{f}}{\delta_{1}}
$$
as[37]:

$$
\left\{\begin{array}{l}
F_{x-l f}=-\left(K-m_{f} \tau_{f}^{2} \omega^{2}\right) x_{02}-\tau_{f} \omega D_{s} y_{02}-D_{s} \&_{2}-2 \tau_{f} m_{f} \omega q_{2}-m_{f} \\
F_{y-l f}=-\left(K-m_{f} \tau_{f}^{2} \omega^{2}\right) y_{02}+\tau_{f} \omega D_{s} x_{02}-D_{s} \&_{2}+2 \tau_{f} m_{f} \omega q_{2}-m_{f}
\end{array}\right.
$$

where $K, D_{s}$, and $\tau_{f}$ are the equivalent stiffness of the sealing forces, the equivalent damping coefficient, and the nonlinear function of displacement perturbation, respectively. Their corresponding expressions are written as follows:

$$
\left\{\begin{array}{l}
K=K_{0}\left(1-e_{t}^{2}\right)^{-j} \\
D_{s}=D_{0}\left(1-e_{t}^{2}\right)^{-j} \\
\tau_{f}=\tau_{0}\left(1-e_{t}\right)^{b_{0}}
\end{array}\right.
$$

in $\mathrm{Eq}(13)$, we have $j=\frac{1}{2} \sim 3,0<b_{0}<1$. The $e_{t}$ is the relative eccentricity of the runner $\left(e_{t}=\sqrt{x_{2}^{2}+y_{2}^{2}} / \delta_{2}, \delta_{2}\right.$ is sealing clearance). Where $j, b_{0}$, and $\tau_{0}$ were used to describe the specific sealing, generally, we have $\tau_{0}<0.5$.

The sealing force was calculated as follows:

$$
K_{0}=\mu_{3} \mu_{0}, \quad D_{0}=\mu_{1} \mu_{3} T, \quad m_{f}=\mu_{2} \mu_{3} T^{2}
$$

The operation is accompanied by arcuate whirl centrifugal force of the generator and hydro-turbine, which were calculated as follows[38]: 


$$
\begin{gathered}
F_{G-G}=\frac{\left(j_{1}+2 m_{1} e_{1}^{2}\right)\left(r_{3}+r_{4}\right) \omega^{2}}{2 g} \\
F_{T-G}=\frac{\left(j_{2}+2 m_{2} e_{2}^{2}\right) \omega^{2} r_{5}}{g}
\end{gathered}
$$

The HTGU is always accompanied by axial hydrodynamic thrust during operation, which is determined by the empirical formula as follow[39]:

$$
F_{z}=K_{z} \frac{\pi}{4} D_{1}^{2} \frac{n \sqrt{Q}}{H^{0.25}}
$$
combined force of the unit's gravity and axial hydraulic thrust, so we have:

$$
F_{G}=\left(m_{11}+m_{22}\right) g+F_{z}
$$

Based on the above supposes, the kinetic energy of the SCV can be written as:

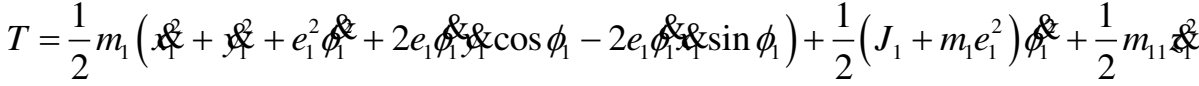

$$
\begin{aligned}
& \frac{1}{2} m_{2}\left(z_{2}+e_{2}^{2} \phi_{2}^{2}+2 e_{2} \phi_{2} \psi_{2} \cos \phi_{2}-2 e_{2} \phi_{2} \psi_{2} \sin \phi_{2}\right)+\frac{1}{2}\left(J_{2}+m_{2} e_{2}^{2}\right) \phi_{2}^{2}+\frac{1}{2} m_{22}
\end{aligned}
$$

in Eq (19), $\left(x_{i}, y_{i}, z_{i}\right), J_{i}, m_{i}, e_{i}, \phi_{i}$, and $\phi_{i}^{\&}=\omega_{i}(i=1,2)$ are the centroid coordinates, the rotational inertia, the equivalent concentrated masses, the eccentricity distance, the rotation angle, and the rotational angular velocity of the generator rotor and the hydro-turbine runner, respectively. 
the HTGU can be expressed as follows:

$$
\begin{array}{r}
U=\left(x_{1}^{2}+y_{1}^{2}\right) K_{11}+\left(x_{2}^{2}+y_{2}^{2}\right) K_{22}+\sqrt{x_{1}^{2}+y_{1}^{2}} \sqrt{x_{2}^{2}+y_{2}^{2}} K_{12}+ \\
\frac{1}{2} K_{y}\left(\phi_{1}-\phi_{2}\right)^{2}+\frac{1}{2}\left(k_{z}+k_{l}\right) z_{1}^{2}+m_{11} g z_{1}+\frac{1}{2} k_{l} z_{2}^{2}+m_{22} g z_{2}
\end{array}
$$

where $K_{11}, K_{22}$, and $K_{12}$ is the equivalent stiffness of the shafting system of the HTGU, $K_{y}$ is the elastic coefficient of the spindle, $k_{l}$ is the axial stiffness, $k_{z}$ is the thrust guide bearing stiffness. $\phi_{1}$ and $\phi_{2}$ are the rotation angles of the generator and the hydro-turbine runner, respectively [19].

$$
\left\{\begin{array}{l}
K_{11}=\frac{A_{1}^{2}}{B^{2}} k_{1}+\frac{(c+d)^{2}}{(b+c+d)^{2}} k_{2}+\frac{d^{2}}{(b+c+d)^{2}} k_{3} \quad(a) \\
K_{22}=\frac{A_{2}^{2}}{B^{2}} k_{1}+\frac{b^{2}}{(b+c+d)^{2}} k_{2}+\frac{(b+c)^{2}}{(b+c+d)^{2}} k_{3} \quad(b) \\
K_{12}=\frac{-A_{1} A_{2}}{B^{2}} k_{1}+\frac{b(c+d)}{(b+c+d)^{2}} k_{2}+\frac{d(b+c)}{(b+c+d)^{2}} k_{3} \quad(c)
\end{array}\right.
$$

in Eq (21), $A_{1}=(a+b)(b+c+d), A_{2}=a b, B=b(b+c+d) \quad k_{1}, k_{2}$, and $k_{3}$ are the supporting stiffness of the upper guide bearing, the lower guide bearing, and the hydro-turbine guide bearing, respectively.

$$
\begin{gathered}
K_{y}=\frac{E \pi\left(d_{H}^{2}-d_{B}^{2}\right)}{32 l} \\
k_{l}=32 K_{y}
\end{gathered}
$$

in $\mathrm{Eq}(22), E$ is the elastic modulus of the spindle. $d_{H}$ and $d_{B}$ are the internal and external diameters of the spindle.

$l$ is the total length of the generator lower spindle and the hydro-turbine spindle.

Assuming that the shafting damping is simplified as linear damping applied to the generator and hydro-turbine runner, and the external excitation force is also considered, then the generalized force of the HTGU can be formulated as follows [32]: 


$$
\left\{\begin{array}{l}
Q_{x i}=-c_{i} x_{i}+F_{x i} \\
Q_{y i}=-c_{i} y_{i}+F_{y i} \\
Q_{z i}=-c_{i} z_{i}+F_{z i}
\end{array}\right.
$$

1 in Eq (24), $c_{i}$ is the damping coefficient, $F_{x i}$ is the excitation force in the horizontal $x$ direction, $F_{y i}$ is the excitation force in the horizontal $y$ direction, and $F_{z i}$ is the excitation force in the horizontal $z$ direction.

$$
\frac{\mathrm{d}}{\mathrm{d} t}\left(\frac{\partial T}{\partial \underset{q_{l}}{\&}}\right)-\left(\frac{\partial T}{\partial q_{i}}\right)+\left(\frac{\partial U}{\partial q_{i}}\right)=Q_{i}
$$

where $T$ is the kinetic energy expressed by various generalized coordinates and generalized velocities of the system.

$U$ is the potential energy represented by the generalized coordinates. $Q_{i}$ is the corresponding generalized force. motion equations of the shafting can be written as:

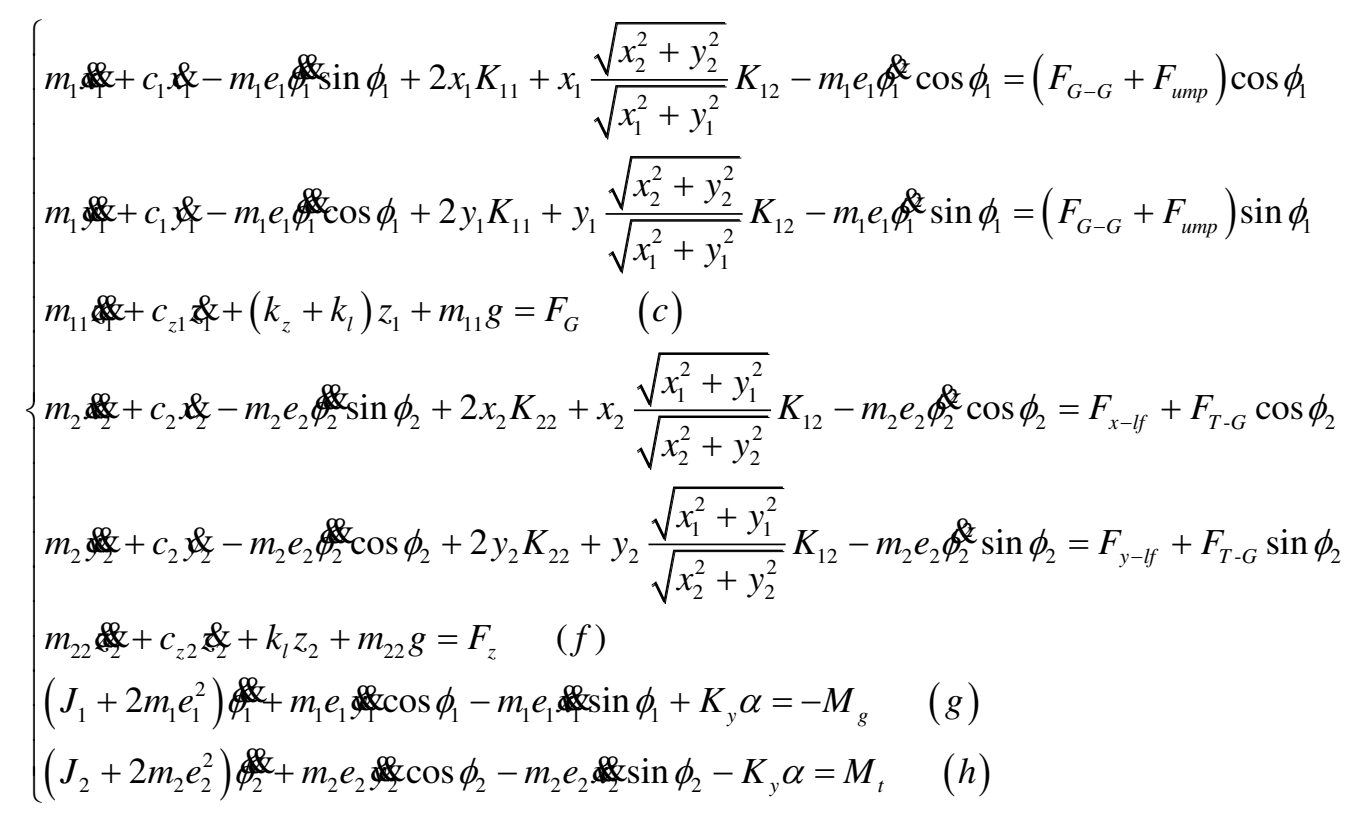


1 the rotational phase of the hydro-turbine runner. we have $\phi_{1}=\int \omega \mathrm{d} t+\varphi_{1}$ and $\phi_{2}=\int \omega \mathrm{d} t+\varphi_{2}$, it can be further obtained that the phase difference between the generator and the hydro-turbine runner is $\alpha=\phi_{1}-\phi_{2}=\varphi_{1}-\varphi_{2}$.

$$
\begin{aligned}
& \left(J_{1}+2 m_{1} e_{1}^{2}\right) \\
& \frac{J K_{y} \alpha}{\left(J_{2}+2 m_{2} e_{2}^{2}\right)\left(J_{1}+2 m_{1} e_{1}^{2}\right)}-\frac{m_{1} e_{1} \cos \phi_{1}-m_{1} e \sin \phi_{1}}{\left(J_{2}+2 m_{2} e_{2}^{2}\right)}-\frac{\left.m_{2} e_{2}\left(J_{2}+2 m_{2} e_{2}^{2}\right)+m_{t}\left(J_{1}+2 m_{1} e_{1}^{2}\right)\right)}{\omega_{m B}\left(J_{1}+2 m_{1} e_{1}^{2}\right)\left(J_{2}+2 m_{2} e_{2}^{2}\right)} \\
& J \frac{\mathrm{d} \omega}{d t}=M_{t}-M_{g}-m_{1} e_{1}\left(\cos \phi_{1}-\sin \phi_{1}\right)-m_{2} e_{2}\left(\cos \phi_{2}-\sin \phi_{2}\right)
\end{aligned}
$$
process of perturbation factor, the Eq (28) can be written as:

$$
J \frac{\mathrm{d} \omega}{\mathrm{d} t}=\left(M_{t}-M_{g}-m_{1} e_{1}\left(\cos \phi_{1}-\sin \phi_{1}\right)-m_{2} e_{2}\left(\cos \phi_{2}-\sin \phi_{2}\right)\right) / \omega_{m B}
$$

7 Due to the $x=\left(n-n_{0}\right) / n_{0}$, and $\omega=\left(n \pi / 30-n_{0} \pi / 30\right) / n_{0} \pi / 30$, so we have $\frac{\mathrm{d} \omega}{\mathrm{d} t}=\frac{\mathrm{d} x}{\mathrm{~d} t}$. $M_{g B}$ is the basic value 8 of the generator torque, $\omega_{m B}$ is the basic value of rotation speed (Where $M_{g B}=S_{g B} / \omega_{m B}, \omega_{m B}=\frac{n_{r} \pi}{30}$ 9 $M_{t}=m_{t} M_{g B}=m_{t} S_{g B} / \omega_{m B}$, and $\left.M_{g}=m_{g} M_{g B}=m_{g} S_{g B} / \omega_{m B}\right)$ The inertia time constant of the HTGU is $T_{a b}=\frac{\left(J_{2}+2 m_{2} e_{2}^{2}+J_{1}+2 m_{1} e_{1}^{2}\right) \omega_{m B}^{2}}{S_{g B}}$. Further, Substituting $J, T_{a b}$ into Eq (29), and rewritten $M_{t}$ and $M_{g}$ in $\mathrm{Eq}(29)$, the motion equation of the HTGU can be written as:

$$
\frac{\mathrm{d} x}{\mathrm{~d} t}=\frac{m_{t}-m_{g}}{T_{a b}}+\frac{\omega_{m B}\left(m_{1} e_{1}\left(\sin \phi_{11}-\cos \phi\right)+m_{2} e_{2}\left(\sin \phi_{2}-\cos \phi_{2}\right)\right)}{T_{a b} S_{g B}}
$$


$\frac{\mathrm{d} x}{\mathrm{~d} t}=\frac{e_{h} \frac{q_{t}-e_{q x} x-e_{q y} y}{e_{q h}}+e_{x} x+e_{y} y-m_{g}-e_{g} x}{T_{a b}}+\frac{\omega_{m B}\left(m_{1} e_{1}\left(\sin \phi_{1}-\cos \phi_{1}\right)+m_{2} e_{2}\left(\sin \phi_{2}-\cos \phi_{2}\right)\right)}{T_{a b} S_{g B}}$

1 in Eq (31), $\phi_{1}=(1+x) \omega_{m B} t, \phi_{2}=(1+x) \omega_{m B} t+\alpha$. nonlinear equation is written as follows:

$$
\begin{aligned}
& \underset{\psi}{\&}=\left(-\frac{q_{t}-e_{q x} x-e_{q y} y}{e_{q h}}-\frac{2 h_{t 0}}{H_{0}} q_{t}\right) / T_{w t} \\
& \&=\frac{e_{h} \frac{q_{t}-e_{q x} x-e_{q y} y}{e_{q h}}+e_{x} x+e_{y} y-m_{g}-e_{g} x}{T_{a b}}+\frac{\omega_{m B}\left(m_{1} e_{1}\left(\sin \phi_{1}-\cos \phi_{1}\right)+m_{2} e_{2}\left(\sin \phi_{2}-\cos \phi_{2}\right)\right)}{T_{a b} S_{g B}} \\
& \&=\frac{u-y}{T_{y}} \\
& \iota \&=\frac{-k_{p}}{T_{a b}}\left(e_{h} \frac{q_{t}-e_{q x} x-e_{q y} y}{e_{q h}}+e_{x} x+e_{y} y-m_{g}-e_{g} x\right)-k_{i} x-\frac{k_{d}}{T_{a b}}\left(\frac{e_{h}}{e_{q h}} \&_{t}+\left(e_{x}-e_{g}-\frac{e_{h} e_{q x}}{e_{q h}}\right) \operatorname{Rt}\left(e_{y}-\frac{e_{h} e_{q y}}{e_{q h}}\right) \& \&\right) \\
& =\left(\left(F_{u m p}+F_{G-G}\right) \cos \phi_{1}+m_{1} e_{1} \& \sin \phi_{1}-c_{1} \&-2 x_{1} K_{11}-x_{1} \frac{\sqrt{x_{2}^{2}+y_{2}^{2}}}{\sqrt{x_{1}^{2}+y_{1}^{2}}} K_{12}+m_{1} e_{1}\left((1+x) \omega_{m B}\right)^{2} \cos \phi_{1}\right) / m_{1} \\
& =\left(\left(F_{u m p}+F_{G-G}\right) \sin \phi_{1}+m_{1} e_{1} \& \cos \phi_{1}-c_{1} \&-2 y_{1} K_{11}-y_{1} \frac{\sqrt{x_{2}^{2}+y_{2}^{2}}}{\sqrt{x_{1}^{2}+y_{1}^{2}}} K_{12}+m_{1} e_{1}\left((1+x) \omega_{m B}\right)^{2} \sin \phi_{1}\right) / m_{1} \\
& =\left(F_{G}-c_{z 1} \&-\left(k_{z}+k_{l}\right) z_{1}-m_{11} g\right) / m_{11} \\
& =\left(F_{x-l f}+F_{T-G} \cos \phi_{2}+m_{2} e_{2} \sin \phi_{2}-c_{2}+2 x_{2} K_{22}-x_{2} \frac{\sqrt{x_{1}^{2}+y_{1}^{2}}}{\sqrt{x_{2}^{2}+y_{2}^{2}}} K_{12}+m_{2} e_{2}\left((1+x) \omega_{m B}\right)^{2} \cos \phi_{2}\right) / m_{2} \\
& =\left(F_{y-l f}+F_{T-G} \sin \phi_{2}+m_{2} e_{2} \operatorname{scos} \phi_{2}-c_{2}-2 y_{2} K_{22}-y_{2} \frac{\sqrt{x_{1}^{2}+y_{1}^{2}}}{\sqrt{x_{2}^{2}+y_{2}^{2}}} K_{12}+m_{2} e_{2}\left((1+x) \omega_{m B}\right)^{2} \sin \phi_{2}\right) / m_{2} \\
& =\left(F_{z}-c_{z 2}-k_{1} z_{2}-m_{22} g\right) / m_{22} \\
& =-c_{t} \&-\frac{m_{1} e_{1} \cos \phi_{1}-m_{1} e_{1} \sin \phi_{1}}{\left(J_{1}+2 m_{1} e_{1}^{2}\right)}+\frac{m_{2} e_{2}}{\left(J_{2}+2 m_{2} e_{2}^{2}\right)}- \\
& K_{y} \alpha \frac{\left(J_{2}+2 m_{2} e_{2}^{2}+J_{1}+2 m_{1} e_{1}^{2}\right)}{\left(J_{2}+2 m_{2} e_{2}^{2}\right)\left(J_{1}+2 m_{1} e_{1}^{2}\right)}-\frac{S_{g B}\left(m_{g}\left(J_{2}+2 m_{2} e_{2}^{2}\right)+m_{t}\left(J_{1}+2 m_{1} e_{1}^{2}\right)\right)}{\omega_{m B}\left(J_{1}+2 m_{1} e_{1}^{2}\right)\left(J_{2}+2 m_{2} e_{2}^{2}\right)}
\end{aligned}
$$

in Eq (32), $c_{t}$ \& is the linear torsional damping force. 
The governor is one of the most important parts of the HTGU. The control parameters of the governor have a very important influence on the regulation performance and stability of the unit. At the same time, PID governor is widely used in engineering practice. Therefore, we focus on the analysis of PID parameters on the stability and sensitivity of the unit. A hydropower station is selected for analyzing the stability and sensitivity of the governor parameter. The classical fourth-order Runge-Kutta method is adopted to solve the nonlinear equation of the coupling HTGS with the shafting system in MATLAB. The actual parameters of a hydropower station are shown in Table 1.

Table 1 Actual parameters of a hydropower station

\begin{tabular}{|c|c|c|c|c|c|}
\hline parameter & value & unit & parameter & value & unit \\
\hline$m_{1}$ & $4.91 \times 10^{5}$ & $k g$ & $a$ & 2.54 & $m$ \\
\hline$m_{2}$ & $5.6 \times 10^{4}$ & $k g$ & $b$ & 2.04 & $m$ \\
\hline$m_{11}$ & $5.65432 \times 10^{5}$ & $k g$ & $c$ & 8.19 & $m$ \\
\hline$m_{22}$ & $1.17 \times 10^{5}$ & $k g$ & $d$ & 1.765 & $m$ \\
\hline$c_{1}$ & $4.1 \times 10^{6}$ & $N \cdot s / m$ & $e_{1}$ & 0.0005 & $m$ \\
\hline$c_{2}$ & $2.52 \times 10^{6}$ & $N \cdot s / m$ & $e_{2}$ & 0.0005 & $m$ \\
\hline$c_{z 1}$ & $3.6 \times 10^{6}$ & $N \cdot s / m$ & $d_{H}$ & 1.15 & $m$ \\
\hline$c_{z 2}$ & $6.31 \times 10^{6}$ & $N \cdot s / m$ & $d_{B}$ & 0.3 & $m$ \\
\hline$c_{t}$ & 1000 & $N \cdot \mathrm{s} / \mathrm{rad}$ & $d_{1}$ & 7.44 & $m$ \\
\hline$k_{1}$ & $1.85 \times 10^{8}$ & $N / m$ & $L$ & 2.927 & $m$ \\
\hline$k_{2}$ & $1.45 \times 10^{8}$ & $N / m$ & $D$ & 7.44 & $m$ \\
\hline$k_{3}$ & $1.15 \times 10^{7}$ & $N / m$ & $\delta_{1}$ & 0.0004 & $m$ \\
\hline$k_{z}$ & $1.27 \times 10^{7}$ & $N / m$ & $\delta_{2}$ & 0.0021 & $m$ \\
\hline$j_{1}$ & $4.625 \times 10^{6}$ & $\mathrm{~kg} \cdot \mathrm{m}^{2}$ & $l$ & 10.3 & $m$ \\
\hline$j_{2}$ & $2 \times 10^{5}$ & $\mathrm{~kg} \cdot \mathrm{m}^{2}$ & $n_{r}$ & 250 & $r / \min$ \\
\hline E & $206 \times 10^{9}$ & $P_{a}$ & $S_{g B}$ & 306 & $M W$ \\
\hline$H_{0}$ & 195 & $m \cdot \mathrm{H}_{2} \mathrm{O}$ & $h_{t 0}$ & 3.2723 & $m$ \\
\hline$Q$ & 176.1 & $m^{3} / s$ & $T_{y}$ & 0.02 & $s$ \\
\hline$e_{x}$ & -1 & & $T_{a b}$ & 10.8072 & $s$ \\
\hline$e_{q h}$ & 0.5 & & $T_{w t}$ & 2.74 & $s$ \\
\hline$e_{q y}$ & 1 & & $\tau_{0}$ & 0.23 & \\
\hline$e_{h}$ & 1.5 & & $D_{1}$ & 5.34 & $m$ \\
\hline$e_{y}$ & 1 & & $B$ & 1 & $T$ \\
\hline$g$ & 9.81 & $m / s^{-2}$ & $\beta$ & 0.5 & \\
\hline$\mu_{2}$ & 0.11 & & $n_{0}$ & 0.066 & \\
\hline$\mu_{0}$ & 0.6 & & $m_{0}$ & -0.25 & \\
\hline$\Delta p$ & $0.5 \times 10^{6}$ & $P_{a}$ & $b_{0}$ & 3.5 & \\
\hline$K_{z}$ & 0.8 & & $T_{0}$ & 0.0657 & \\
\hline$R a_{0}$ & 2703 & & $r_{a}$ & 1.86 & $m$ \\
\hline
\end{tabular}


of load disturbance is 0.1 or -0.1 , the stability and sensitivity of the PID parameters to the HTGS and shafting

system vibrations are obtained using the bifurcation diagram. Since the deviation of the characteristic quantity of the HTGS is consistent, the deviation of the rotation speed is selected as the typical characteristic variable of the HTGS.

3.1 The stability and sensitivity of $k_{p}$ to the rotation speed deviation and shafting system vibrations

The stability and sensitivity of the rotation speed deviation and shafting system vibrations to $k_{p}$ are shown in Fig.3. From Fig.3(a), it is seen that when $k_{p}$ is $[0.1,1.65)$, the rotation speed deviation is bifurcated which shows a strong sensitivity. When $k_{p}$ is $[1.65,5.4]$, the rotation speed deviation is stable. When $k_{p}$ is $(5.4,5.6]$, the rotation speed deviation is chaotic that shows a strong sensitivity. As can be seen from Fig.3(b), When $k_{p}$ is $[0.1,5.6]$, the negative torsion vibration angles are stable. From Fig.3(c), it is seen that when $k_{p}$ is $[0.1,2)$, the generator vibration of $x$ direction is chaotic that shows obvious vibration and strong sensitivity. When $k_{p}$ is $[2,4.05]$, the generator vibration of $x$ direction is quasi-periodic which shows slight vibration, and when $k_{p}$ is $[4.05,5.35]$, the generator vibration of $x$ direction still shows quasi-periodic but vibration amplitude increase obviously. When $k_{p}$ is $(5.35,5.6]$, the generator vibration of $x$ direction is chaotic that shows obvious vibration and strong sensitivity. As can be seen from Fig.3(d), when $k_{p}$ is $[0.1,2.15)$, the generator vibration of $y$ direction is chaotic that shows obvious vibration and strong sensitivity. When $k_{p}$ is $[2.15,3.2]$, the generator vibration of $y$ direction is quasi-periodic which shows slight vibration, and When $k_{p}$ is $[3.2,5.3]$, the generator vibration of $y$ direction still shows quasi-periodic but vibration amplitude increase obviously. When $k_{p}$ is $(5.3,5.6]$, the generator vibration of $y$ direction is chaotic that shows obvious vibration and strong sensitivity. As can be seen from Fig.3(e), when $k_{p}$ is $[0.1,1.35)$, the generator vibration of $z$ direction is bifurcated which shows slight vibration and slight sensitivity. When $k_{p}$ is $[1.35,5.35]$, the generator vibration of $z$ direction is periodic which shows slight vibration. When $k_{p}$ is $(5.35,5.6]$, the generator vibration of $z$ direction is chaotic that shows obvious vibration and strong sensitivity. As can be seen from Fig.3(f) and $(\mathrm{g})$, when $k_{p}$ is $[0.1,1)$, the hydro-turbine vibration of $x$ and $y$ direction is chaotic that shows obvious vibration and strong sensitivity. When $k_{p}$ is $[1,5.4]$, the hydro-turbine vibration of $x$ and $y$ direction is quasi-periodic which 
shows slight vibration. When $k_{p}$ is $(5.4,5.6]$, the hydro-turbine vibration of $x$ and $y$ direction is chaotic that shows obvious vibration and strong sensitivity. From Fig.3(h), it is seen that when $k_{p}$ is $[0.1,1.35)$, the hydro-turbine

3 vibration of $z$ direction is bifurcated which shows obvious vibration and strong sensitivity. When $k_{p}$ is $[1.35,5.3]$, the

4 hydro-turbine vibration of $y$ direction is periodic which shows slight vibration. When $k_{p}$ is $(5.3,5.6]$, the hydro-turbine vibration of $y$ direction is chaotic that shows obvious vibration and strong sensitivity. Meanwhile, under the different load disturbances, the shafting radial vibration shows almost identical stability, However, the axial vibration shows the same stability of $k_{p}$ but the larger amplitude was generated by smaller the load.

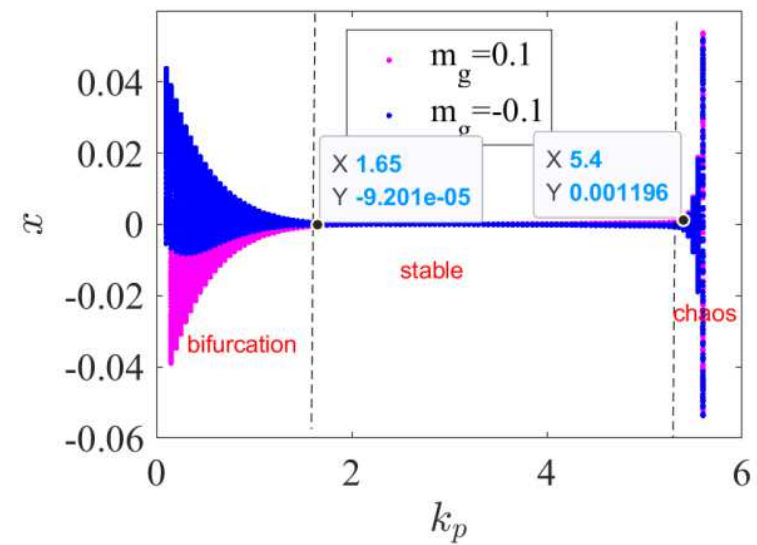

(a) Rotation speed deviation bifurcation diagram of $k_{p}$

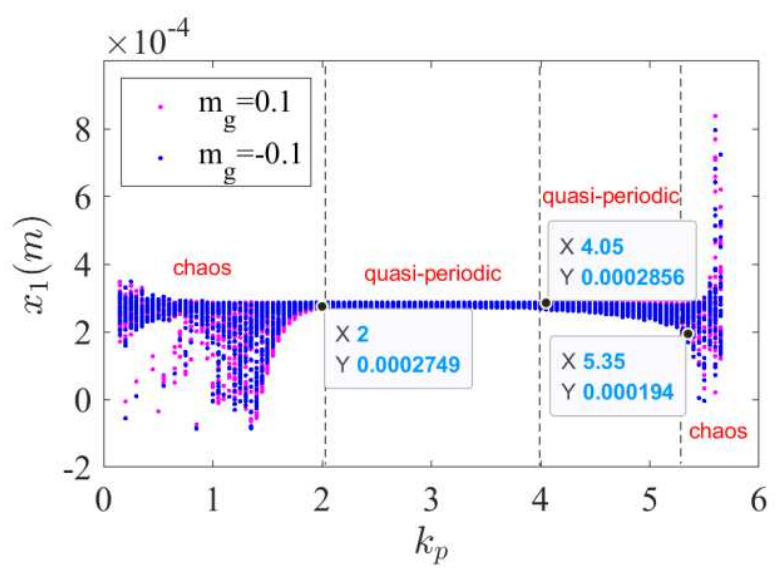

(c) Generator vibration ( $\left.x_{1}\right)$ bifurcation diagram of $k_{p}$

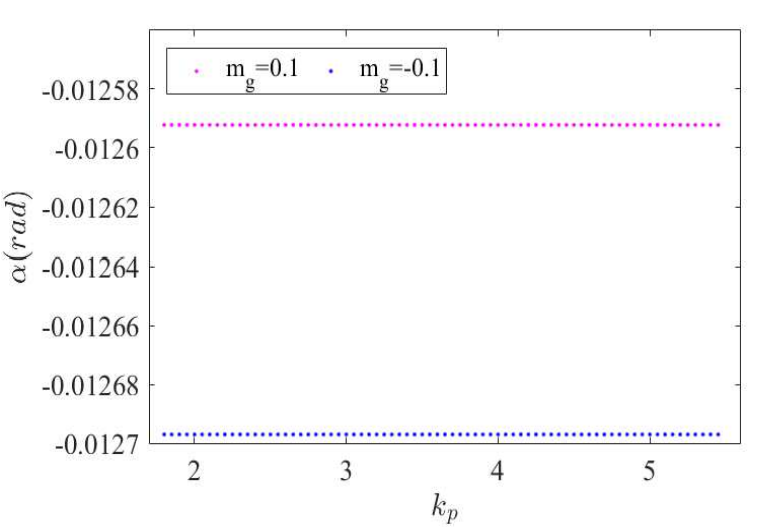

(b) Torsion angle bifurcation diagram of $k_{p}$

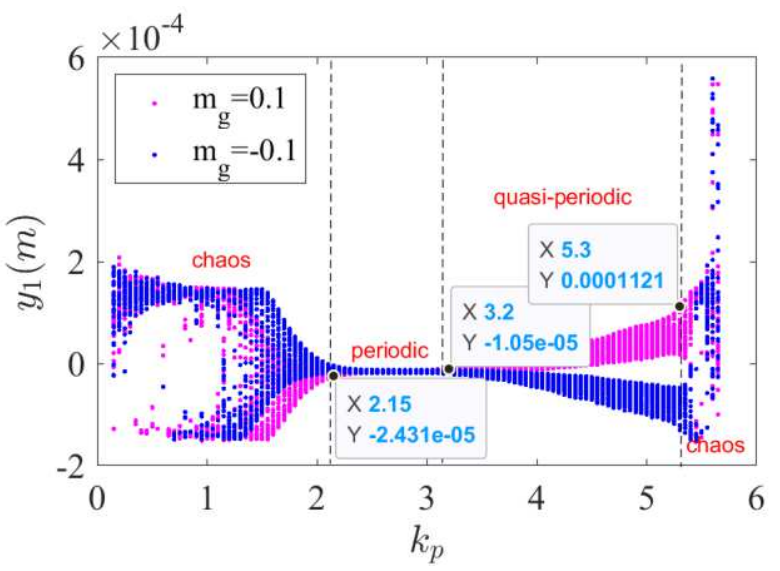

(d) Generator vibration ( $y_{1}$ ) bifurcation diagram of $k_{p}$ 


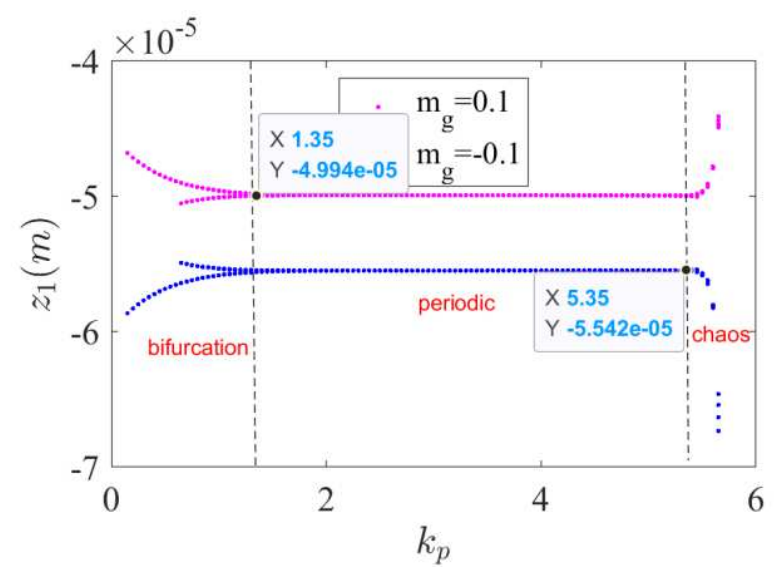

(e) Generator vibration $\left(z_{1}\right)$ bifurcation diagram of $k_{p}$

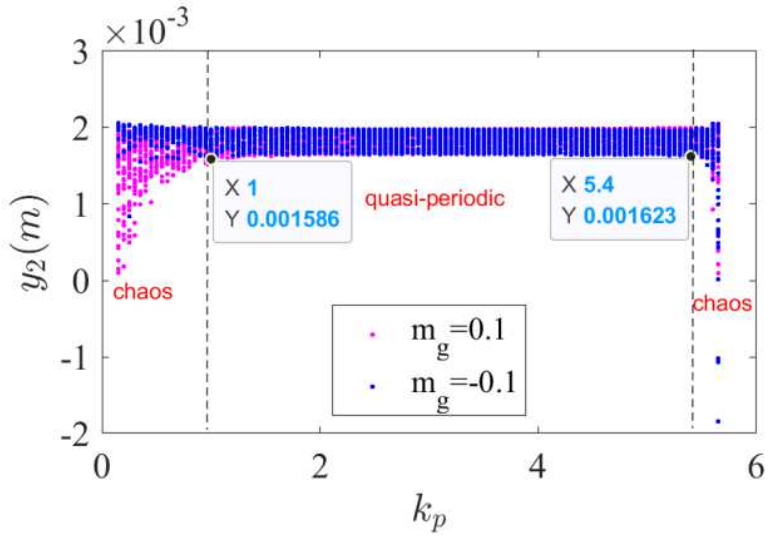

(g) Hydro-turbine vibration $\left(y_{2}\right)$ bifurcation diagram of $k_{p}$

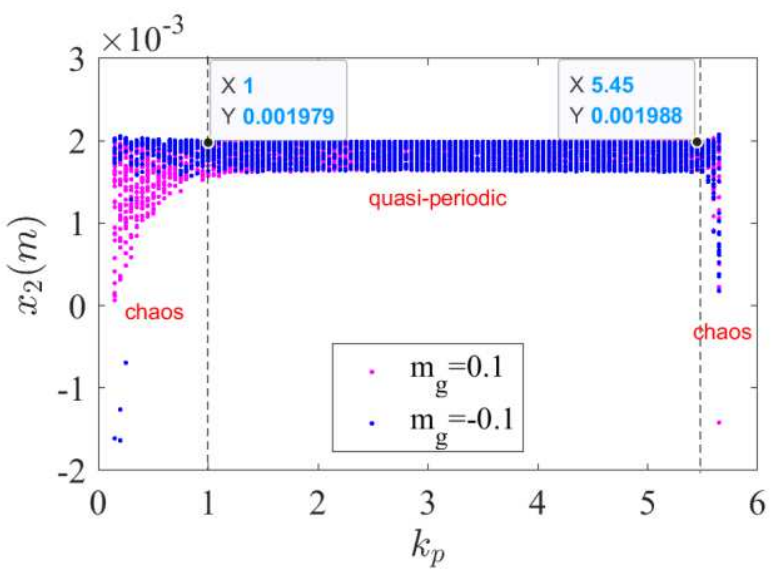

(f) Hydro-turbine vibration $\left(x_{2}\right)$ bifurcation diagram of $k_{p}$

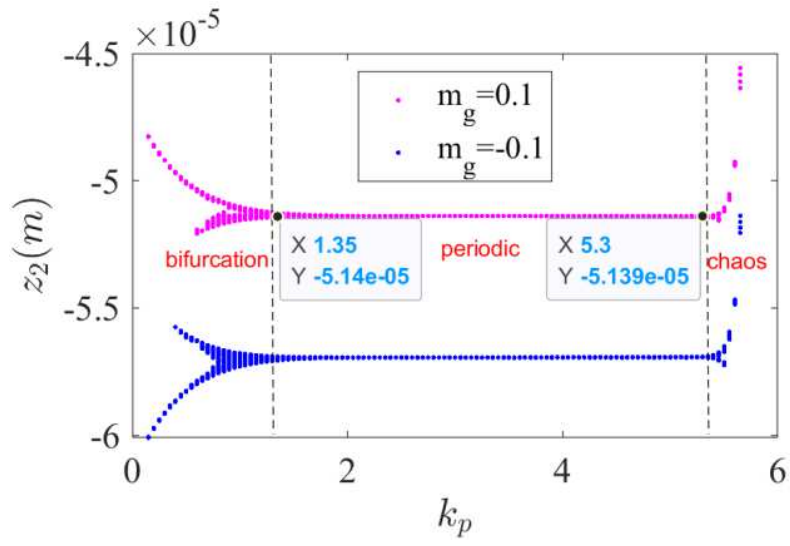

(h) Hydro-turbine vibration $\left(z_{2}\right)$ bifurcation diagram of $k_{p}$ Fig.3 The rotation speed deviation and SCV dynamic characteristics to $k_{p}$

The stability and sensitivity of $k_{i}$ to the rotation speed deviation and shafting system vibrations are shown in Fig.4. rotation speed deviation is bifurcated which shows strong sensitivity. As can be seen from Fig.4(b), When $k_{i}$ is $[0.1,1.15]$, the negative torsion vibration of HTGU is stable. From Fig.4(c), it is seen that when $k_{i}$ is $[0.1,0.21)$, the generator vibration of $x$ direction is chaotic that shows obvious vibration and strong sensitivity. When $k_{i}$ is $[0.21,0.72]$, the generator vibration of $x$ direction is quasi-periodic which shows slight vibration. When $k_{i}$ is $(0.72,1.15]$, the generator vibration of $x$ direction is chaotic that shows obvious vibration and strong sensitivity. As can be seen from Fig.4(d), when $k_{i}$ is $[0.1,0.26)$, the generator vibration of $y$ direction is chaotic that shows obvious vibration and strong sensitivity. When $k_{i}$ is $[0.26,0.59]$, the generator vibration of $y$ direction is quasi-periodic which shows slight vibration. When $k_{i}$ is $(0.59,1.15]$, the generator vibration of $y$ direction is chaotic 
that shows obvious vibration and strong sensitivity. As can be seen from Fig.4(e), when $k_{i}$ is $[0.1,0.83)$, the generator vibration of $z$ direction is periodic which shows slight vibration. When $k_{i}$ is $(0.83,1.15]$, the generator vibration of $z$ direction is bifurcated which shows obvious vibration and strong sensitivity. As can be seen from Fig.4(f) and (g), when $k_{i}$ is $[0.1,0.89)$, the hydro-turbine vibration of $x$ and $y$ direction is quasi-periodic which shows slight vibration. When $k_{i}$ is $[0.89,1.15]$, the hydro-turbine vibration of $x$ and $y$ direction is chaotic that shows obvious vibration and strong sensitivity. From Fig.4(h), it is seen that when $k_{i}$ is $[0.1,0.86)$, the hydro-turbine vibration of $y$ direction is periodic which shows slight vibration. When $k_{i}$ is $[0.86,1.15]$, the hydro-turbine vibration of $y$ direction is chaotic that shows obvious vibration and strong sensitivity. Obviously, under the different load disturbances, the shafting radial vibration shows almost identical stability, However, the axial vibration shows the same

bifurcated characteristics of $k_{i}$ but the smaller amplitude was generated by a larger load.

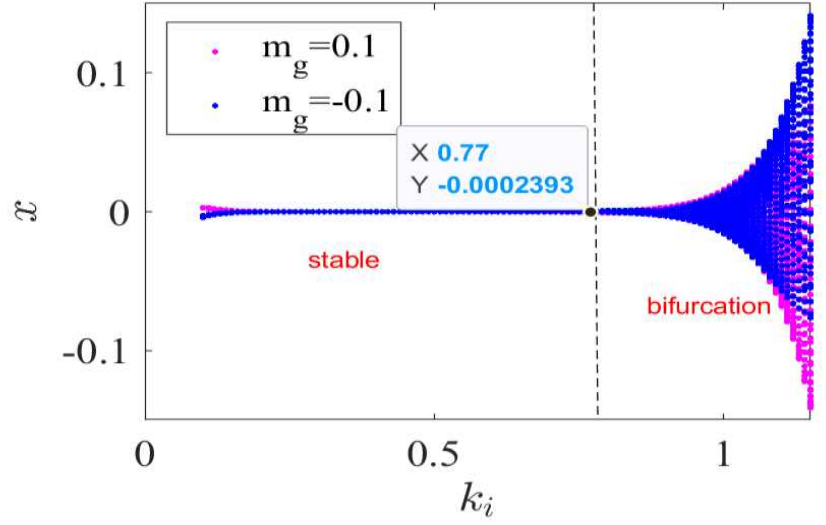

(a) Rotation speed deviation bifurcation diagram of $k_{i}$

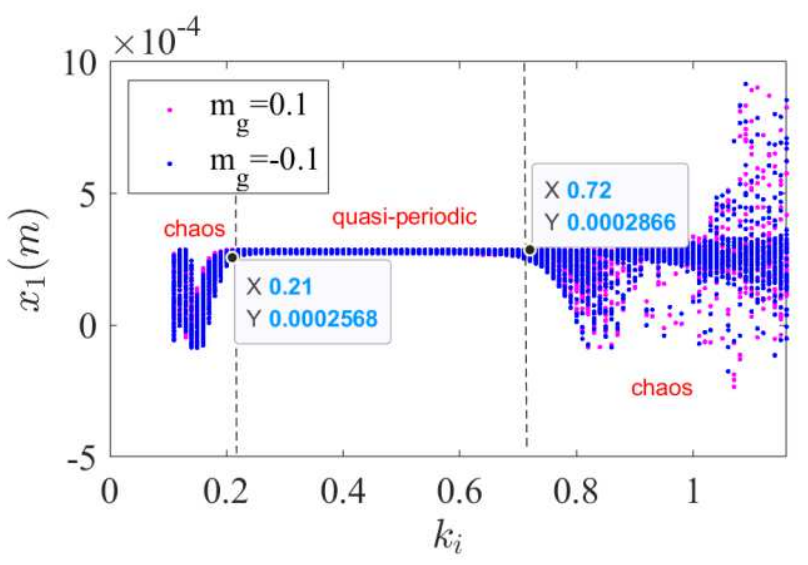

(c) Generator vibration $\left(x_{1}\right)$ bifurcation diagram of $k_{i}$

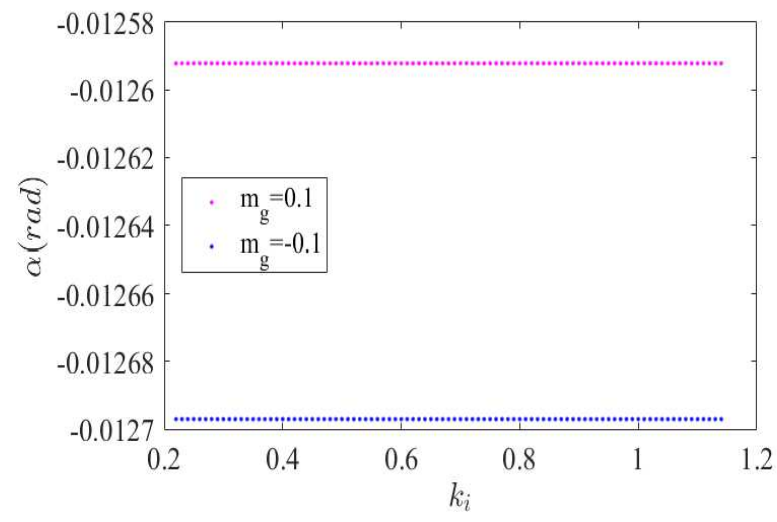

(b) Torsion angle bifurcation diagram of $k_{i}$

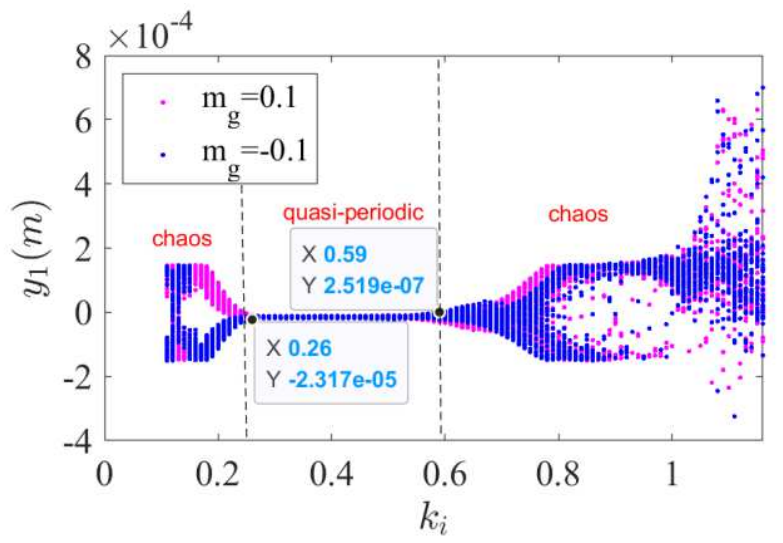

(d) Generator vibration ( $y_{1}$ ) bifurcation diagram of $k_{i}$ 


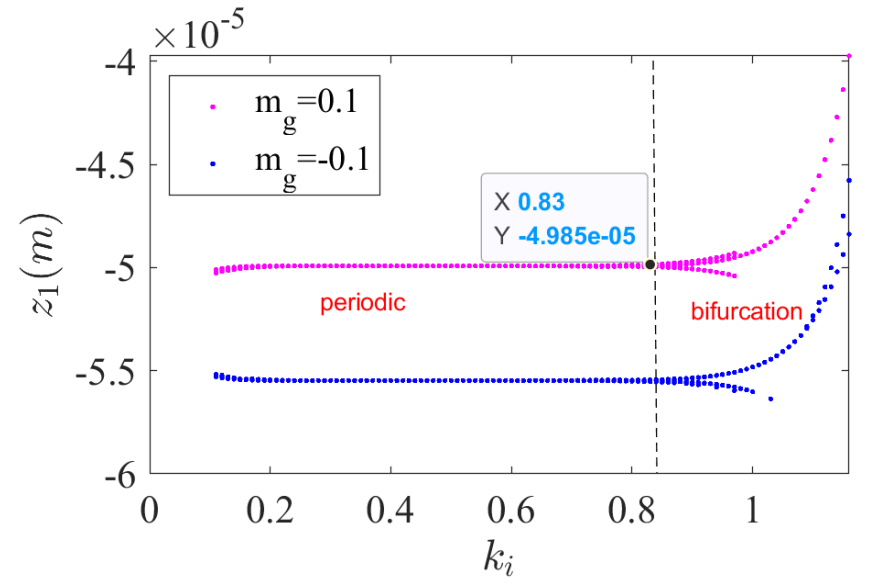

(e) Generator vibration $\left(z_{1}\right)$ bifurcation diagram of $k_{i}$

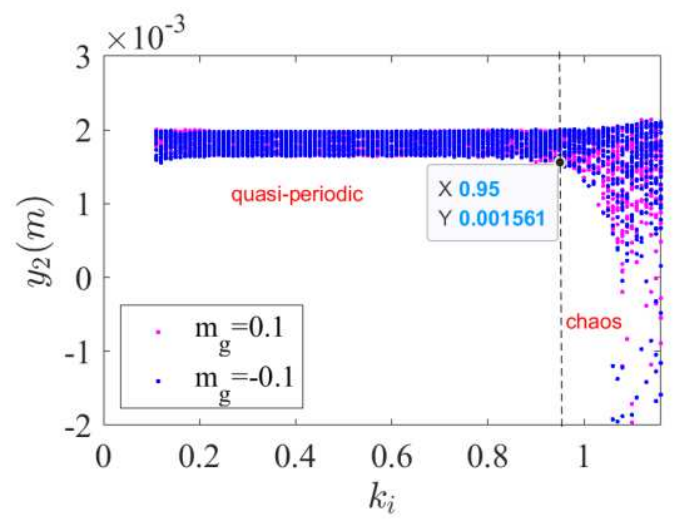

(g) Hydro-turbine vibration $\left(y_{2}\right)$ bifurcation diagram of $k_{i}$

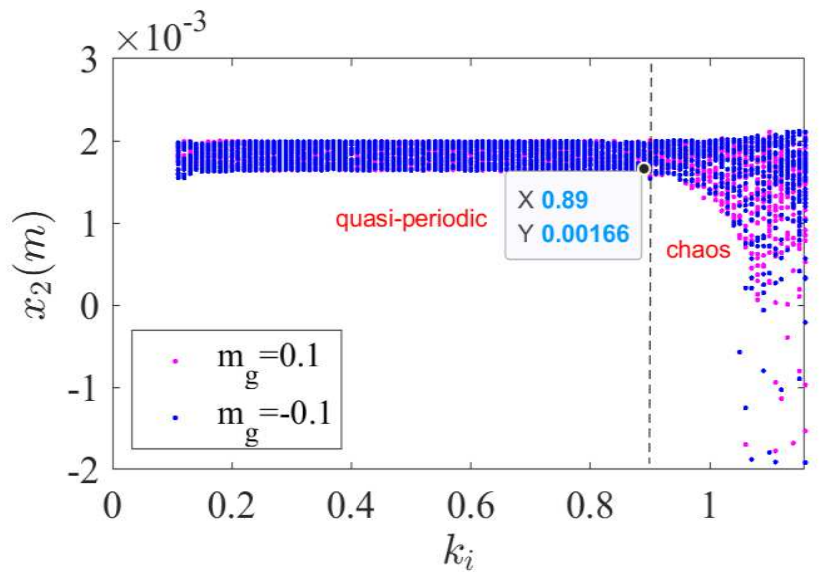

(f) Hydro-turbine vibration $\left(x_{2}\right)$ bifurcation diagram of $k_{i}$

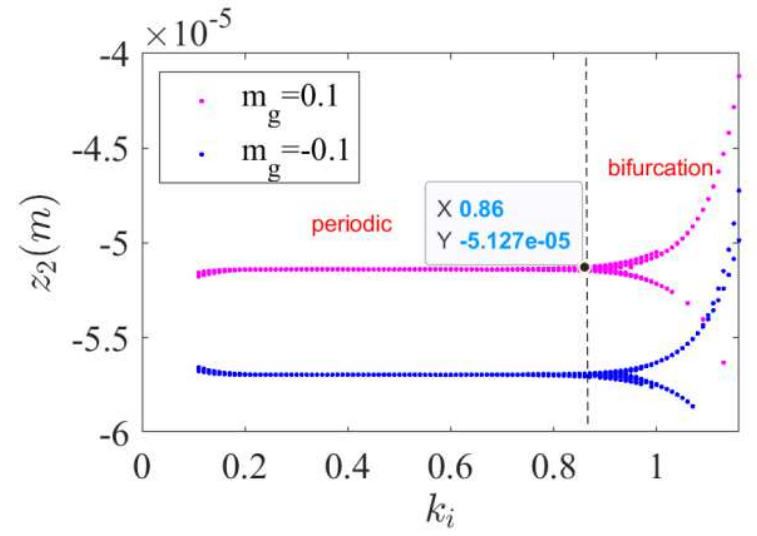

Fig. 4 The rotation speed deviation and SCV dynamic characteristics of $k_{i}$

$1 \quad 3.3$ The stability and sensitivity of $k_{d}$ to the rotation speed deviation and shafting system vibrations

The stability and sensitivity of $k_{d}$ to the rotation speed deviation and shafting system vibrations are shown in Fig.5.

From Fig.5(a), it is seen that when $k_{d}$ is $[0.1,5.48)$, the rotation speed deviation is stable. When $k_{d}$ is $(5.48,5.5]$, the

rotation speed deviation is chaotic that shows a strong sensitivity. As can be seen from Fig.5(b), When $k_{d}$ is $[0.1,5.5]$,

the negative torsion vibration of HTGU is stable. From Fig.5(c), it is seen that when $k_{d}$ is $[0.1,5.46)$, the generator of $x$ direction is chaotic that shows obvious vibration and strong sensitivity. As can be seen from Fig.5(d),

when $k_{d}$ is $[0.1,5.46)$, the generator vibration of $y$ direction is quasi-periodic which shows slight vibration, but when $k_{d}$ is $[0.1,1.78)$ and $[3.62,5.46)$, it shows the slight sensitivity. When $k_{d}$ is $(5.46,5.5]$, the generator vibration 
is $[5.44,5.5]$, the generator vibration of $z$ direction is chaotic that shows obvious vibration and strong sensitivity. As can be seen from Fig.5(f) and (g), when $k_{d}$ is $[0.1,0.5 .46)$, the hydro-turbine vibration of $x$ and $y$ direction is

3 quasi-periodic that shows slight vibration. When $k_{d}$ is $[5.46,5.5]$, the hydro-turbine vibration of $x$ and $y$ direction is 4 chaotic that shows obvious vibration and strong sensitivity. From Fig.5(h), it is seen that when $k_{d}$ is $[0.1,5.48)$, the 5 hydro-turbine vibration of $y$ direction is periodic which shows slight vibration. When $k_{d}$ is $(5.48,5.5]$, the 6 hydro-turbine vibration of $y$ direction is chaotic that shows obvious vibration and strong sensitivity. Obviously, under the different load disturbances, the shafting vibration shows almost identical stability of $k_{d}$, However, the axial vibration's larger amplitude was generated by a smaller load.

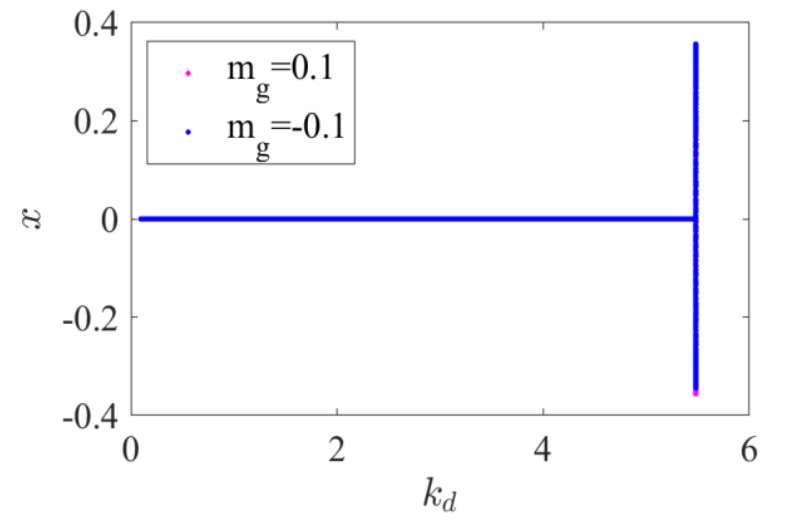

(a) Rotation speed deviation bifurcation diagram of $k_{d}$

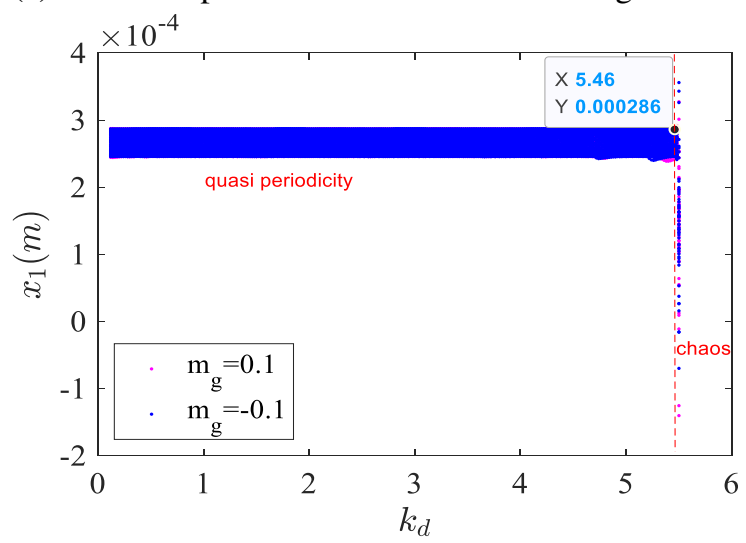

(c) Generator vibration ( $\left.x_{1}\right)$ bifurcation diagram of $k_{d}$

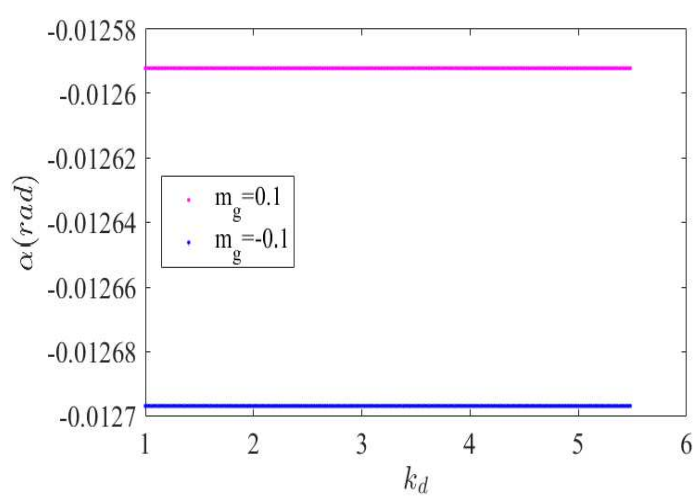

(b) Torsion angle bifurcation diagram of $k_{d}$

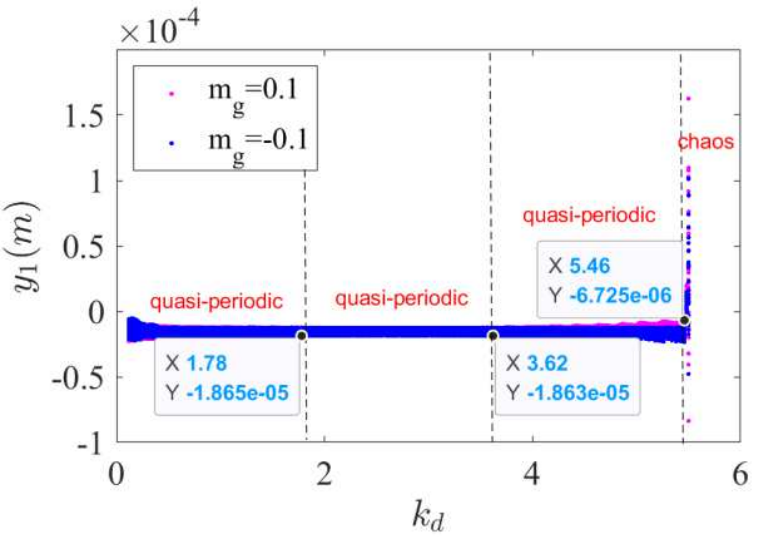

(d) Generator vibration ( $\left.y_{1}\right)$ bifurcation diagram of $k_{d}$ 


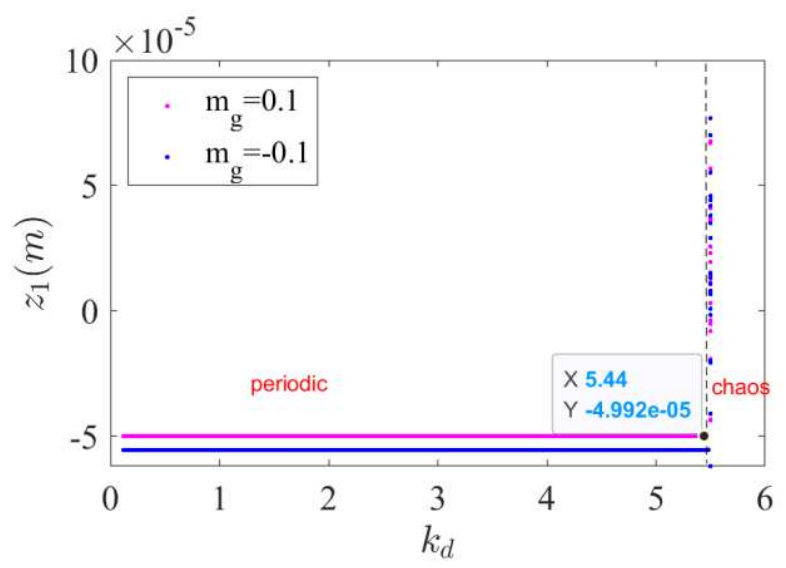

(e) Generator vibration $\left(z_{1}\right)$ bifurcation diagram of $k_{d}$

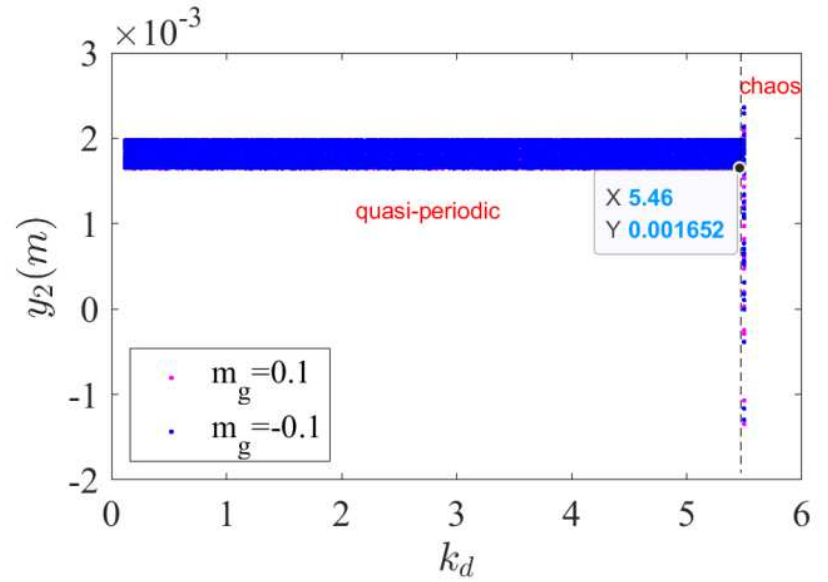

(g) Hydro-turbine vibration $\left(y_{2}\right)$ bifurcation diagram of $k_{d}$

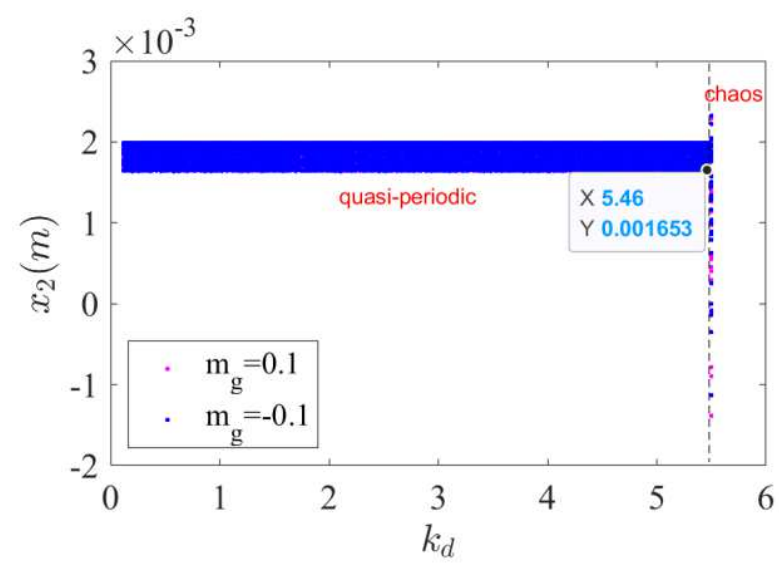

(f) Hydro-turbine vibration ( $\left.x_{2}\right)$ bifurcation diagram of $k_{d}$

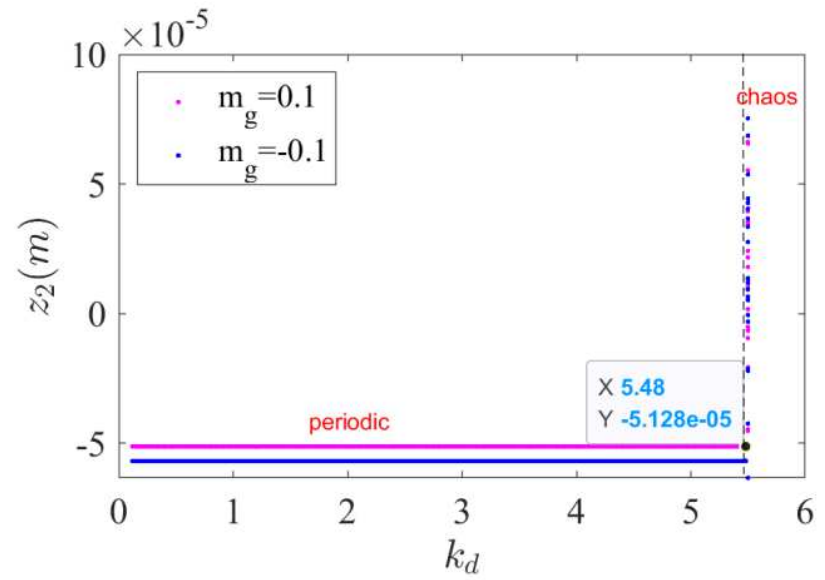

(h) Hydro-turbine vibration $\left(z_{2}\right)$ bifurcation diagram of $k_{d}$ Fig. 5 The rotation speed deviation and SCV dynamic characteristics to $k_{d}$

\section{The governor PID parameter multi-objective optimization control}

Based on the coupling of HTGS with shafting system nonlinear mathematical model, a multi-objective

optimization strategy of PID parameters is proposed with the following innovations: (1) The objective functions consider the rotation speed deviation, the opening of the guide vane, and the shafting system vibrations simultaneously. (2) The objective function is composed of the ITSE. (3) The NSGA-II[41] and MOEAD[42] which have shown high efficiency and competitive performance in solving the multi-objective optimization problems compared with other evolutionary algorithms were introduced.

\subsection{Objective functions}

In the process of load regulation, the rotation speed, guide vane opening, and shafting system vibration will be volatile. Under small load disturbance, to make the complex coupled system to obtain the response output overshoot 
minimize and the shortest regulation time. The ITSE (ITSE $\left.=\int_{0}^{\infty} t e^{2}(t) \mathrm{d} t\right)$ of rotation speed deviation, guide vane

opening deviation, vibration radius deviation of the generator and hydro-turbine in 3D space are selected to establishes the objective functions, which was written as follows:

$$
\left\{\begin{array}{l}
J_{1}=W_{1} \cdot I T S E_{x}+I T S E_{r 11}+I T S E_{r 22} \\
J_{2}=W_{2} \cdot I T S E_{y}+I T S E_{r 11}+I T S E_{r 22}
\end{array}\right.
$$

$\operatorname{In} \mathrm{Eq}(33), W_{1}=W_{2}=2, \quad r_{11}=\sqrt{\left(x_{1}^{2}+y_{1}^{2}+z_{1}^{2}\right)}$, and $r_{22}=\sqrt{\left(x_{2}^{2}+y_{2}^{2}+z_{2}^{2}\right)}$.

\subsection{Decision variables}

When load disturbance occurs to the HTGU, improper PID parameter combination may lead to the whole system slow stable response, or even goes into an unstable or chaotic state, which will lead to control failure of the whole system and lead to power grid oscillation and abnormal vibration of the HTGU. Therefore, we select the parallel PID parameters $k_{p}, k_{i}$, and $k_{d}$ as the decision variables. In section 3, the rotation speed deviation and shafting system vibration stability and sensitivity of $k_{p}, k_{i}$, and $k_{d}$ are given, which can be concluded that the system has different stability domains for different characteristic variables of $k_{p}, k_{i}$, and $k_{d}$. There, we define the periodic and quasi-periodic vibration regions of shafting system vibrations and the stable region of HTGS to form the relative stability region. Therefore, the intersection principle was used to obtain the common relative stable domain consider the shafting system vibration of the parameters of $k_{p}, k_{i}$, and $k_{d}$, and then the boundary of the common stability domain is taken as the boundary of the decision variables. so we have:

$$
\left\{\begin{array}{l}
k_{p s}=k_{p x s} \cap k_{p \alpha s} \cap k_{p x_{1} s} \cap k_{p y_{1} s} \cap k_{p z_{1} s} \cap k_{p x_{2} s} \cap k_{p y_{2} s} \cap k_{p z_{2} s} \\
k_{i s}=k_{i x s} \cap k_{i \alpha s} \cap k_{i x_{1} s} \cap k_{i y_{1} s} \cap k_{i z_{1} s} \cap k_{i x_{2} s} \cap k_{i y_{2} s} \cap k_{i z_{2} s} \\
k_{d s}=k_{d x s} \cap k_{d \alpha s} \cap k_{d x_{1} s} \cap k_{d y_{1} s} \cap k_{d z_{1} s} \cap k_{d x_{2} s} \cap k_{d y_{2} s} \cap k_{d z_{2} s}
\end{array}\right.
$$

Thus, the relative stable domain that considers the HTGS and the shafting system of the decision variables are $k_{p s}=[2.15,3.2], \quad k_{i s}=[0.26,0.59]$, and $k_{d s}=[1.78,3.62]$, respectively.

\subsection{Multi-objective optimization}

According to the above determined objective functions and decision variable boundary, the NSGA-II and MOEAD 
multi-objective optimization algorithms were used to establish the optimization process as shown in Fig.6, and the

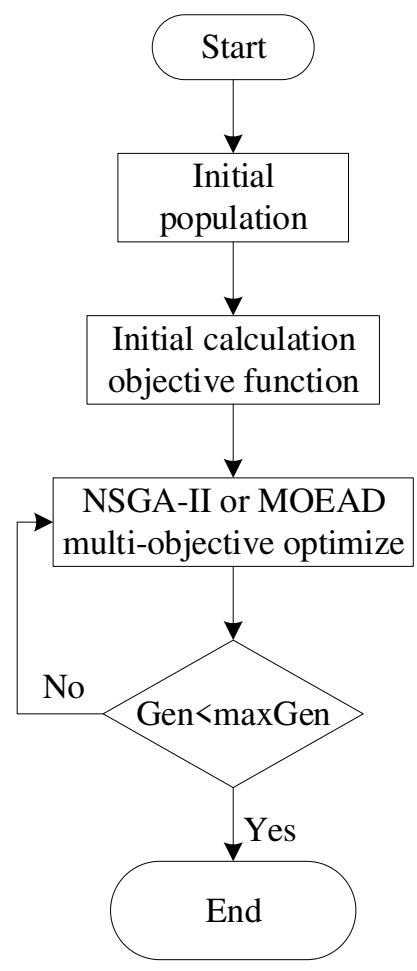

Fig 6 Optimization process diagram

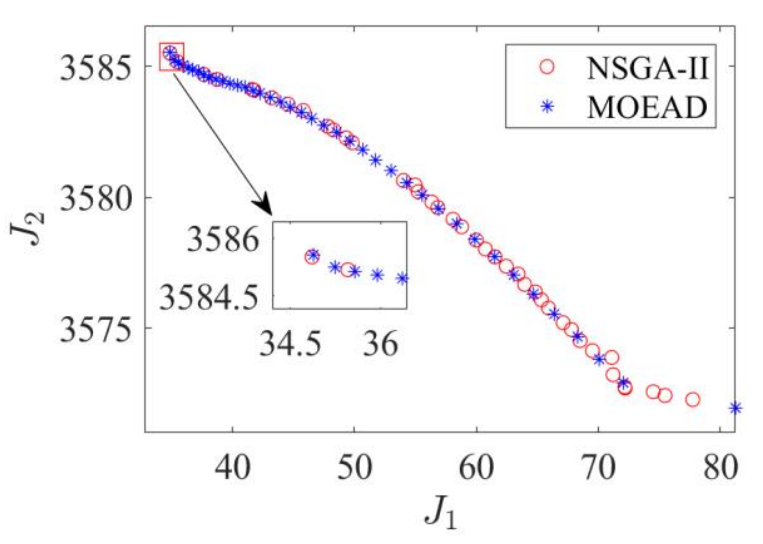

(a) $\mathrm{Mg}=-0.1$

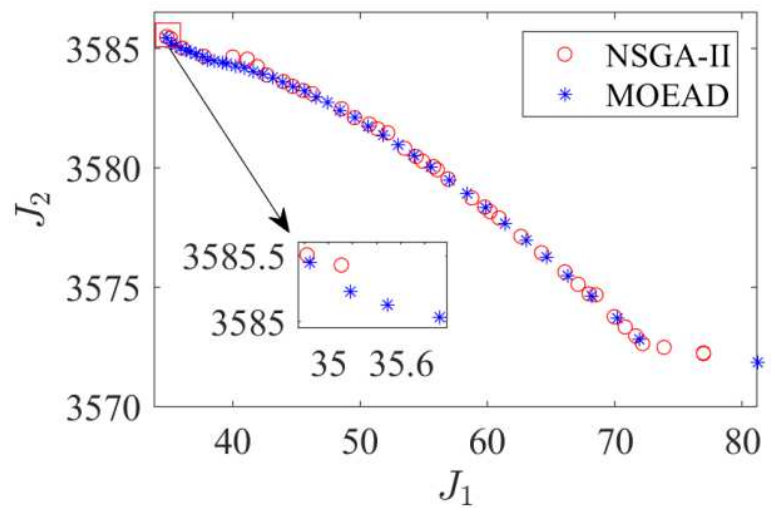

(b) $\mathrm{Mg}=0.1$

Fig 7. Pareto optimal solution obtained by different algorithms

Table 2 the optimal results

\begin{tabular}{ccccccc}
\hline algorithms & Load disturbance & $J_{1}$ & $J_{2}$ & $k_{p}$ & $k_{i}$ & $k_{d}$ \\
\hline NSGA-II & $m_{g}= \pm 0.1$ & 34.9 & 3585.5 & 3.2 & 0.49 & 2.69 \\
MOEAD & $m_{g}= \pm 0.1$ & & & & & \\
\hline
\end{tabular}


In this section, the nonlinear dynamic characteristics of the HTGS, generator vibration, hydro-turbine vibration, and torsion vibration were revealed using the time domain feature, phase trajectory, Poincare map, and frequency spectrum while the small load disturbance happened with the optimal PID control parameters.

5.1 Nonlinear dynamic characteristics of the HTGU to the self-regulating coefficient

The nonlinear dynamic characteristics of the HTGS are shown in Fig.8. As can be seen from Fig.8(a) and (b), while the load disturbance is $m_{g}= \pm 0.1$, the optimized PID parameters make the adjustment time of $q_{t}, x$, and $y$ were

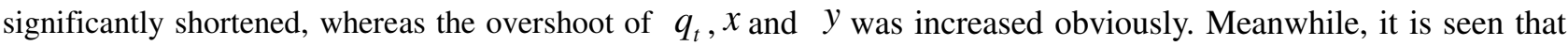
the overshoot of $q_{t}, x$, and $y$ was decreased gradually with the self-regulation coefficient increased.
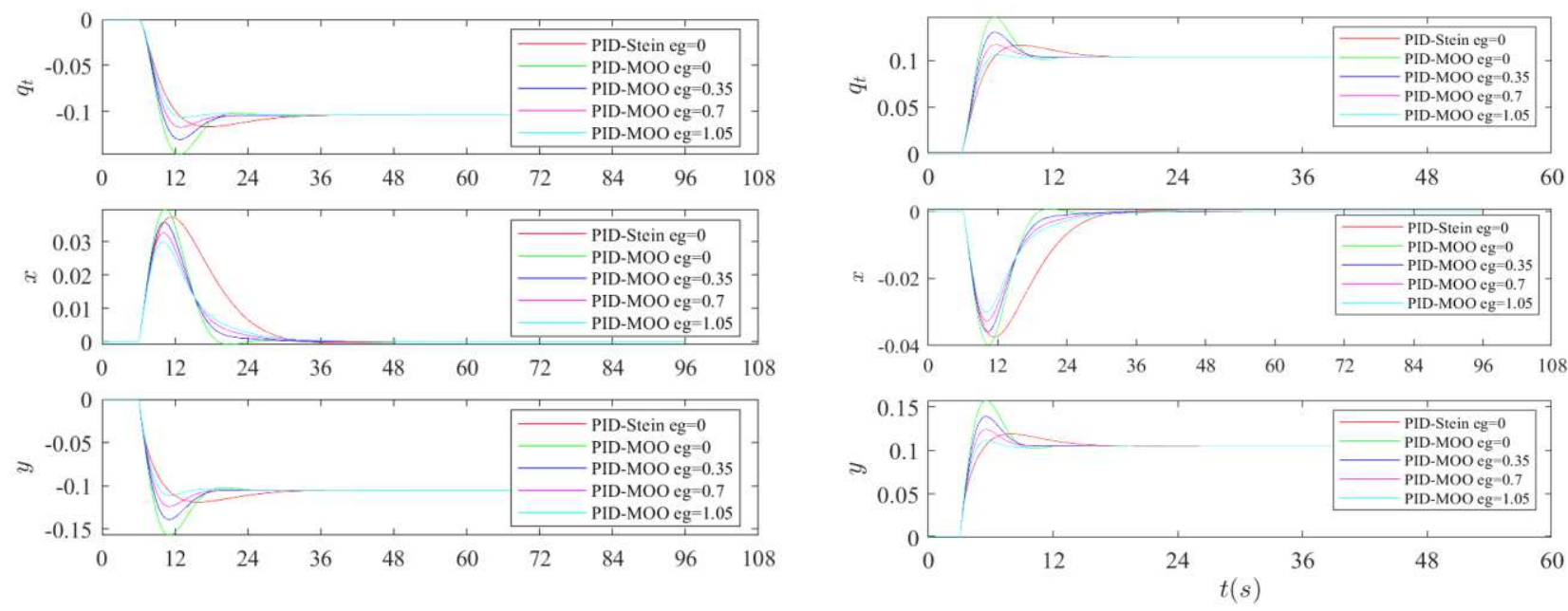
(a) $m_{g}=-0.1$
(b) $m_{g}=0.1$

Fig. 8 The nonlinear dynamic characteristics of the HTGS and (b), while the load disturbance is $m_{g}= \pm 0.1$ the optimized PID parameters make the adjustment time was significantly shortened and the vibration amplitude decreased slightly. Meanwhile, it is seen that the dithering amplitude of $r_{11}$ was decreased gradually with the self-regulation coefficient increased, however, the dithering time is increased slightly. 


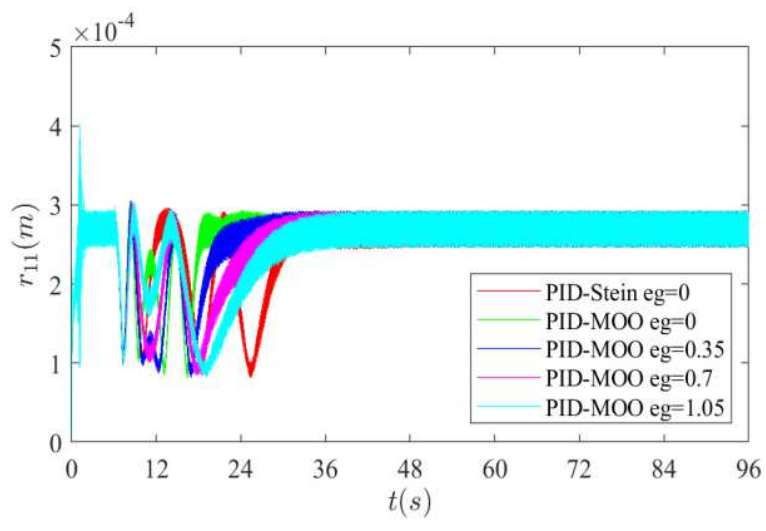

(a) $m_{g}=-0.1$

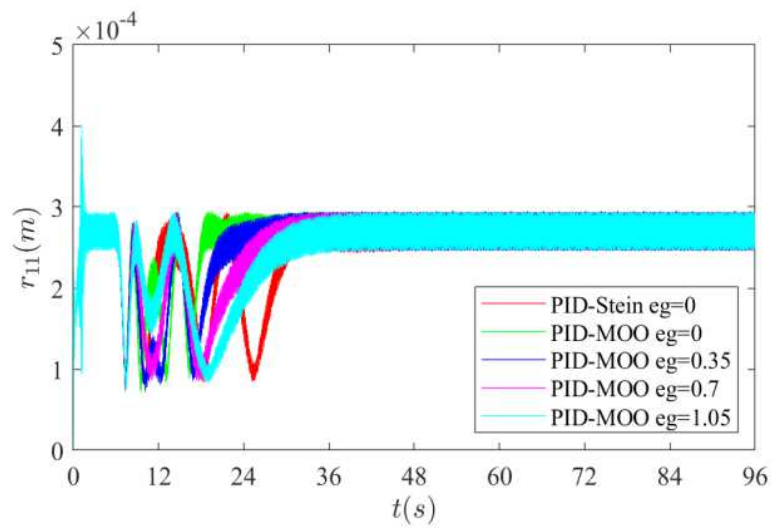

(b) $m_{g}=0.1$

Fig 9 Robustness of optimized PID controller for $r_{11}$ to the load-self regulation coefficient
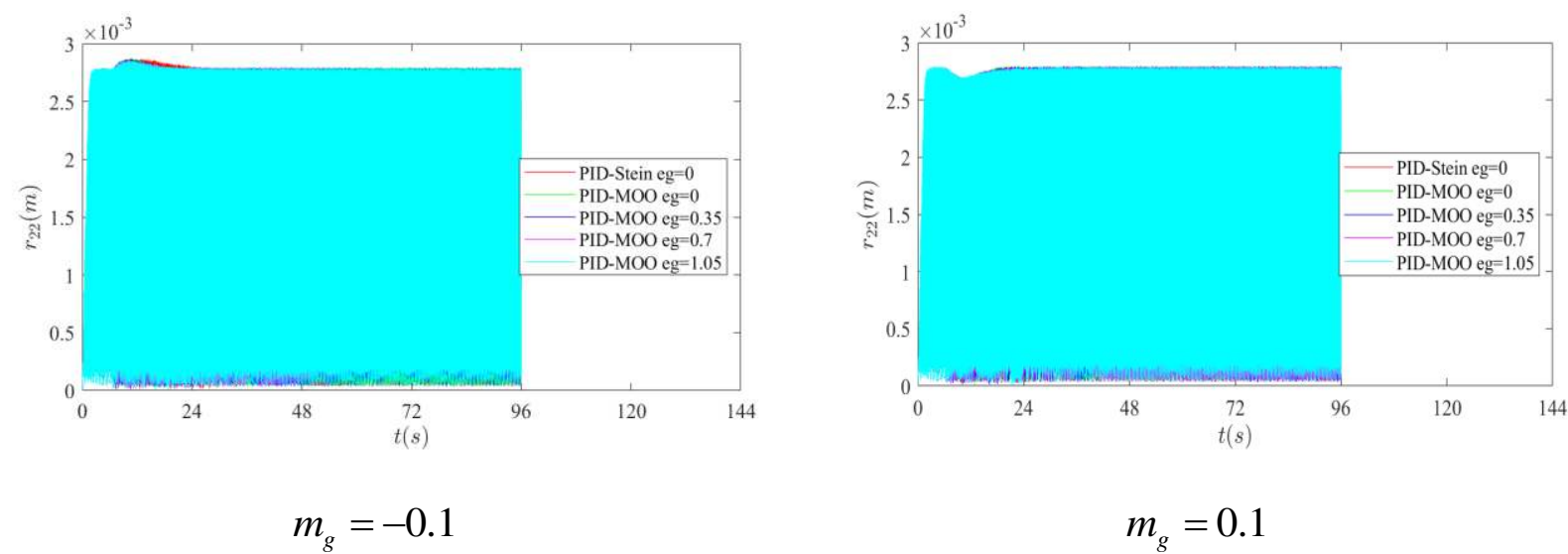

Fig 10 Robustness of optimized PID controller for $r_{22}$ to the load-self regulation coefficient

The dynamic characteristics of spindle torsion vibration are shown in Fig.11. As can be seen from Fig.11(a) and (b), while the load disturbance is $m_{g}= \pm 0.1$ the shafting torsion vibration is stable. Meanwhile, it is seen that the torsion vibration remains same with the self-regulation coefficient increased. 

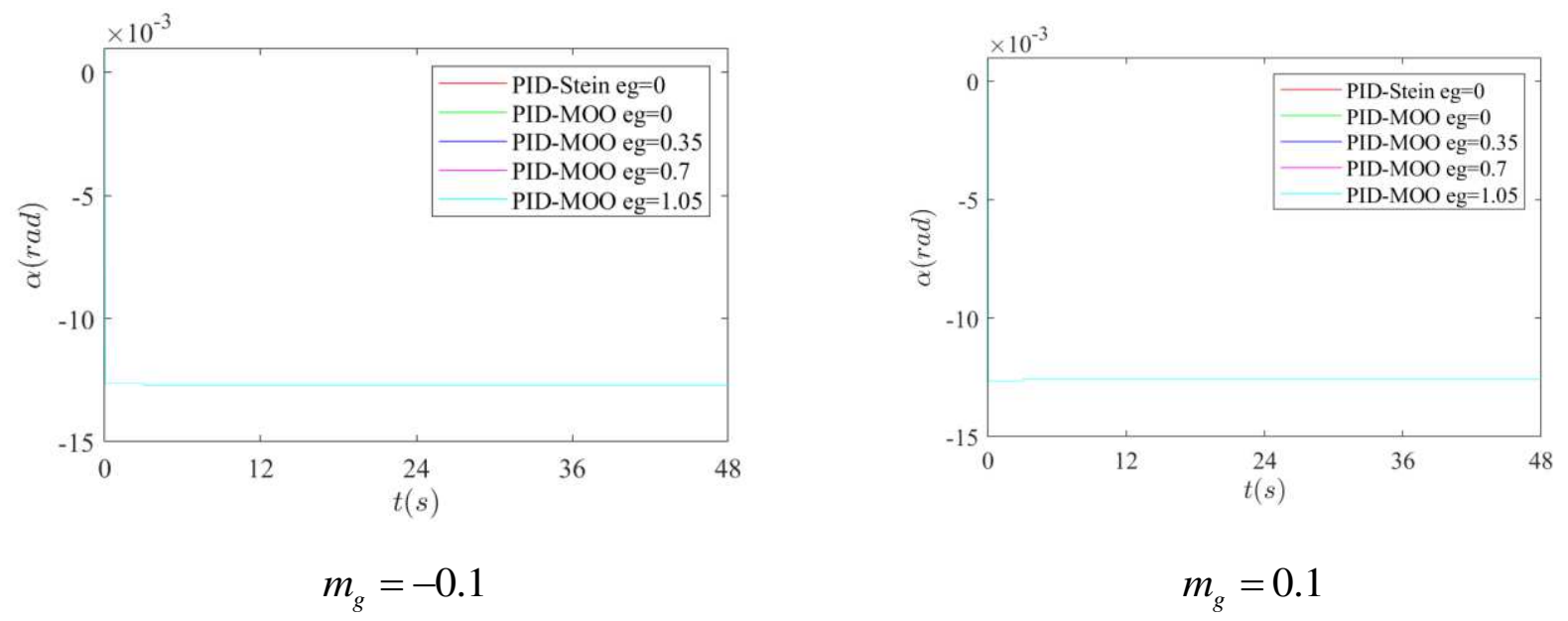

Fig.11 Robustness of optimized PID controller for torsion angle to the load-self regulation coefficient

$1 \quad 5.2$ Nonlinear dynamic characteristics of shafting system vibrations

According to the above research, the nonlinear dynamic characteristics of the shafting system vibrations excited by the mass eccentricity, the UMP, the arcuate whirl of the rotor, and the seal excitation coupled vibration sources, controlled with the optimal PID parameters, and self-regulation coefficient is 1.05 are analyzed. vibration axis locus is multiple closed curves in 3D space, which shows quasi-periodic vibration. As can be seen from Fig.12 (b), (c), (d), (e), (f), and (g), the generator vibration axis locus are multiple closed curves in $x-y$, $x-z, y-z$ 2D space, and the Poincare section show the attractors are a finite number, which can qualitatively analyze that the hydro-turbine vibration of $x-y, x-z, y-z$ 2D space are quasi-periodic. As can be seen from Fig.12 (h) and (1), during load disturbance, the generation vibration of $x$ and $y$ direction go through three fluctuations: first, it decreases rapidly and then increases rapidly; second, it decreases slightly and then increases; finally, it decreases rapidly and then increases rapidly and stable in a certain area. As can be seen from Fig.12 (i), (j), (m), and (n) the generator vibration of $x$ and $y$ direction velocity displacement phase plane are multiple closed curves and the velocity displacement Poincare section show the attractors are a finite number, which can qualitatively analyze that the generator vibration of $x$ and $y$ direction are quasi-periodic. As can be seen from Fig.12(k) and (o), the generator vibration of $x$ and $y$ directions vibration is accompanied by three main vibration frequency components, namely, 

vibration of $z$ direction changes with the corresponding change of load and stable in a certain area, which can be analyzed qualitatively the vibration and the load of the HTGU stay the positive correlation. As can be seen from Fig.12 (q) and (r) the generator vibration of $z$ direction velocity displacement phase plan are multiple closed curves and the velocity displacement Poincare section shows the attractors are a finite number, which can qualitatively analyze that the generator vibration of $z$ direction is quasi-periodic. As can be seen from Fig.12(s), the generator vibration of $z$ direction is accompanied by 0.1 octaves. To sum up the above, it can be seen that the vibration of the generator of $x$ and $y$ directions has almost the same vibration characteristics.

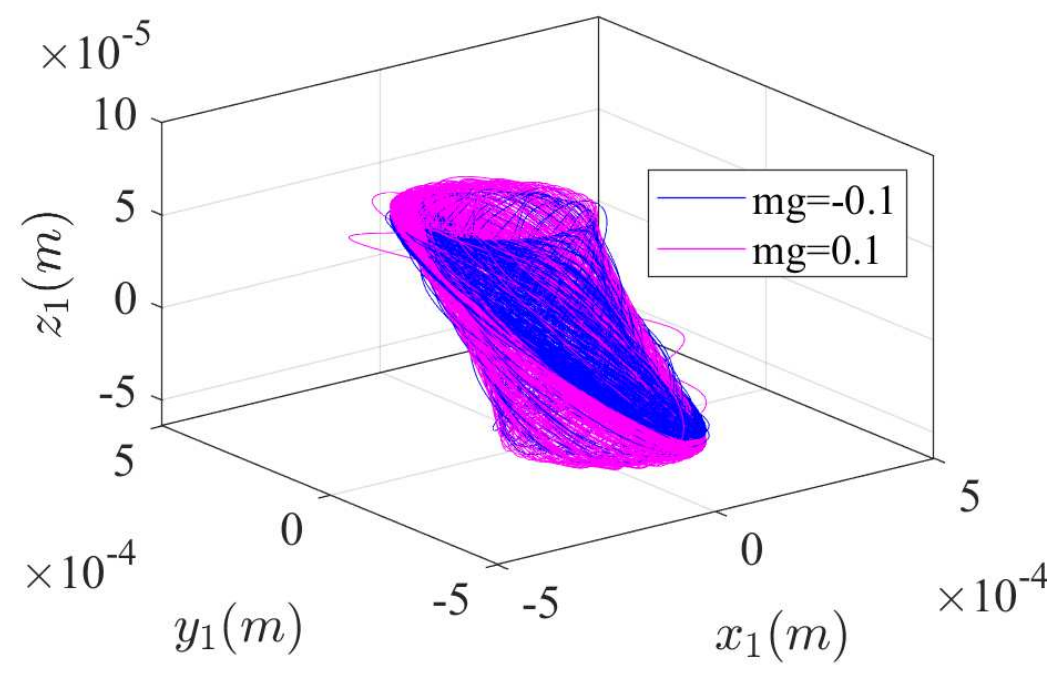

(a) Three-dimensional axis locus of generator vibration

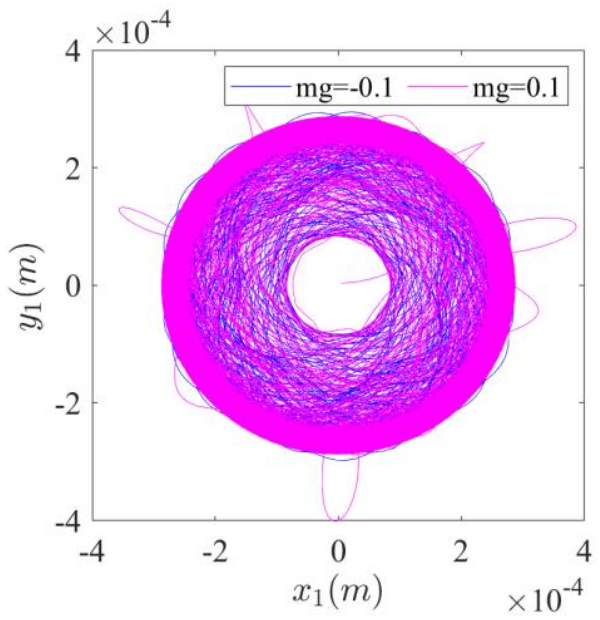

(b) The axis locus of $x-y$ direction

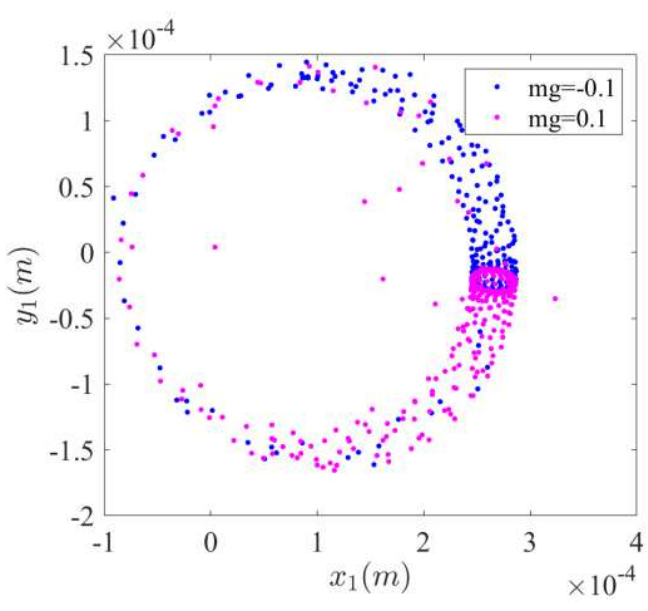

(c)The Poincare section of $x-y$ direction 


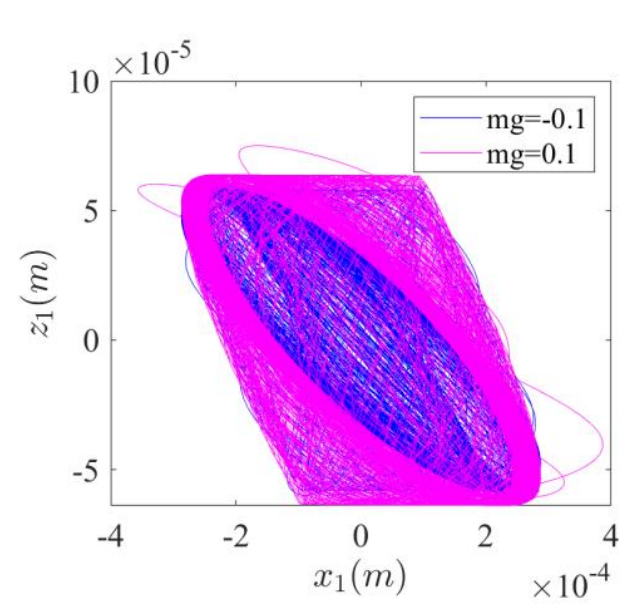

(d) The axis locus of $x-z$ direction

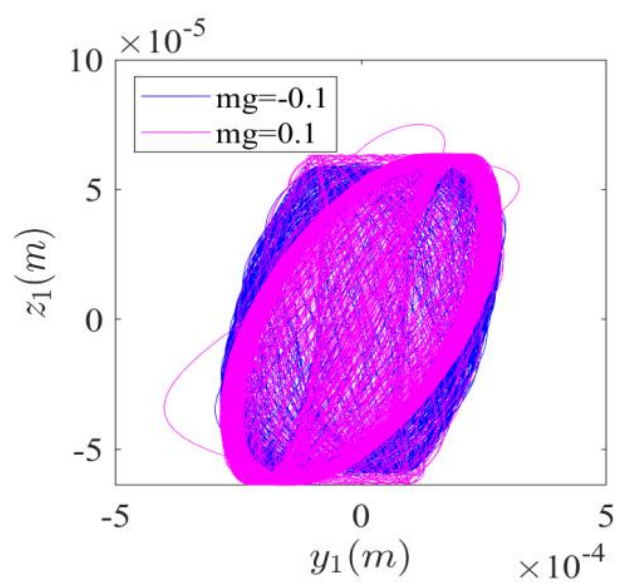

(f) Thel axis locus of $y-z$ direction

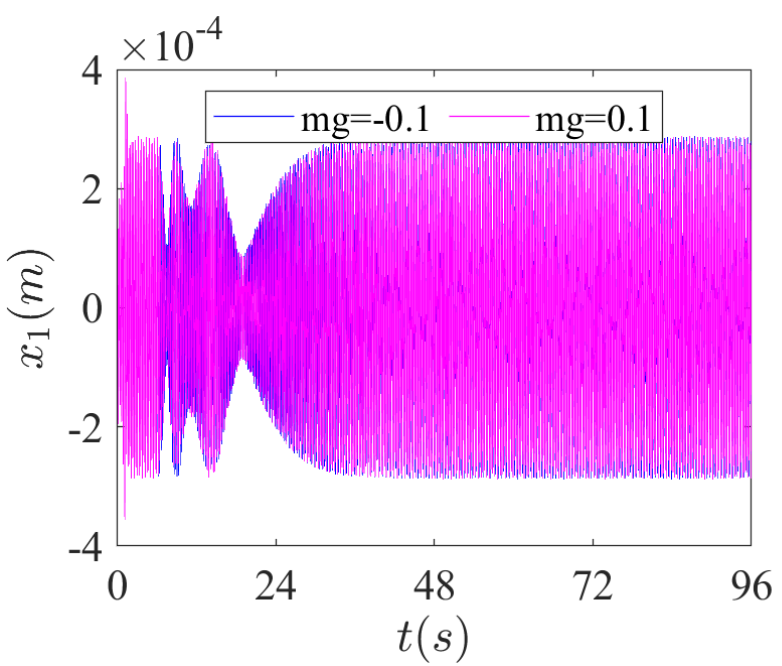

(h) The time-domain diagram of the $x$ direction

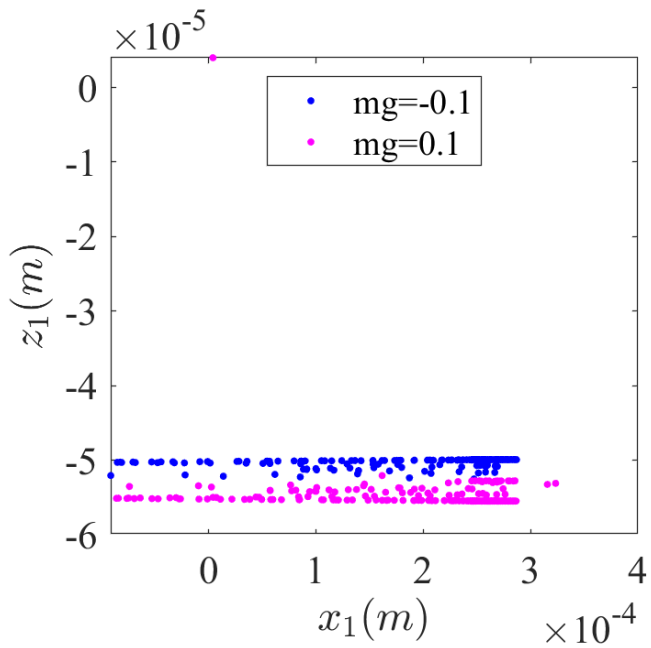

(e) The Poincare section of $x-z$ direction

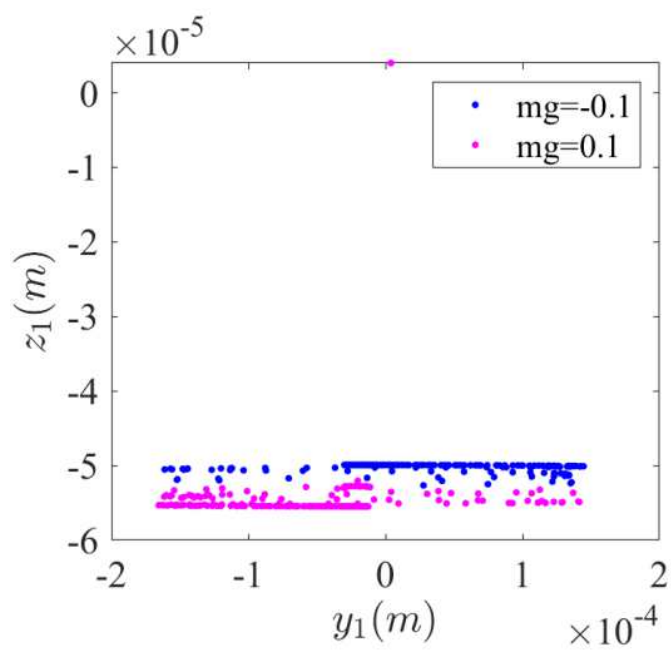

(g) The Poincare section of $y-z$ direction

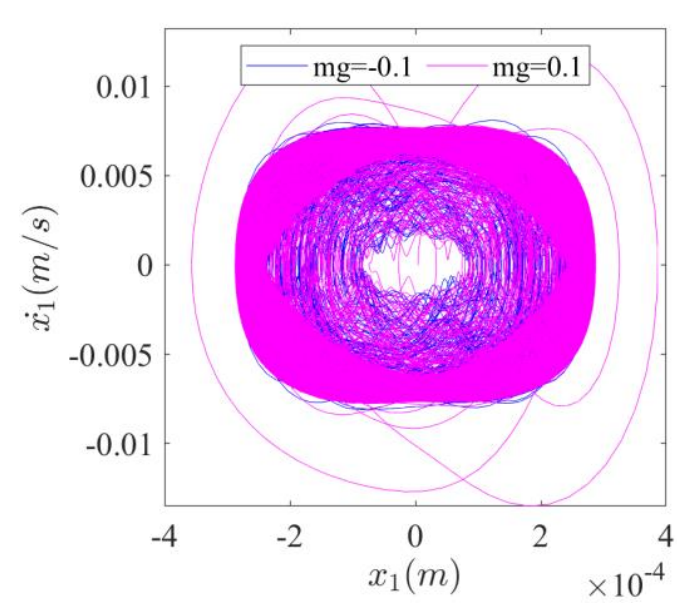

(i) The velocity displacement phase plan of $x$ direction 


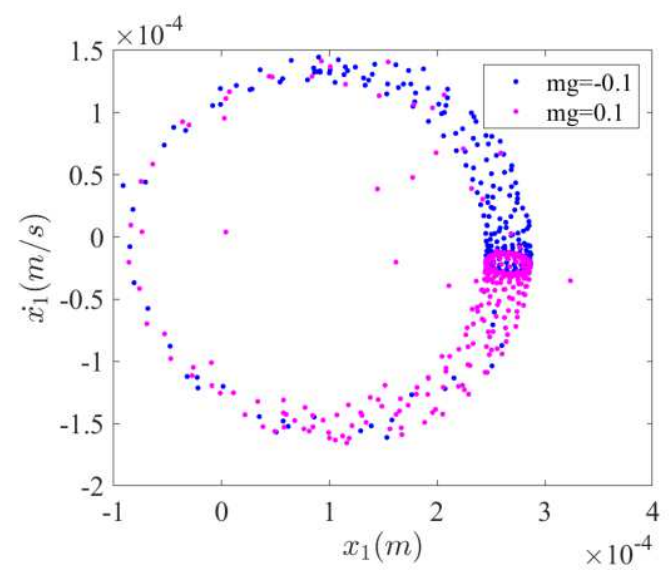

(j) The velocity displacement Poincare section of $x$ direction

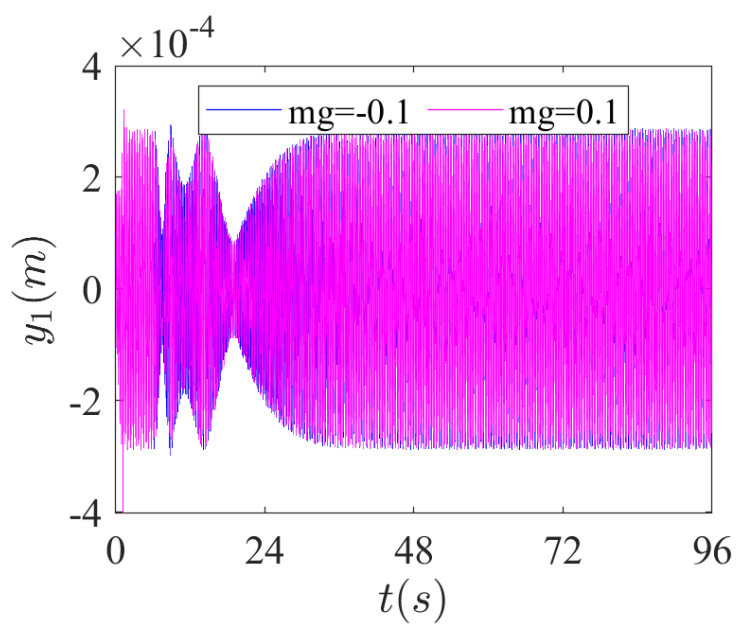

(1) The time-domain diagram of $y$ direction

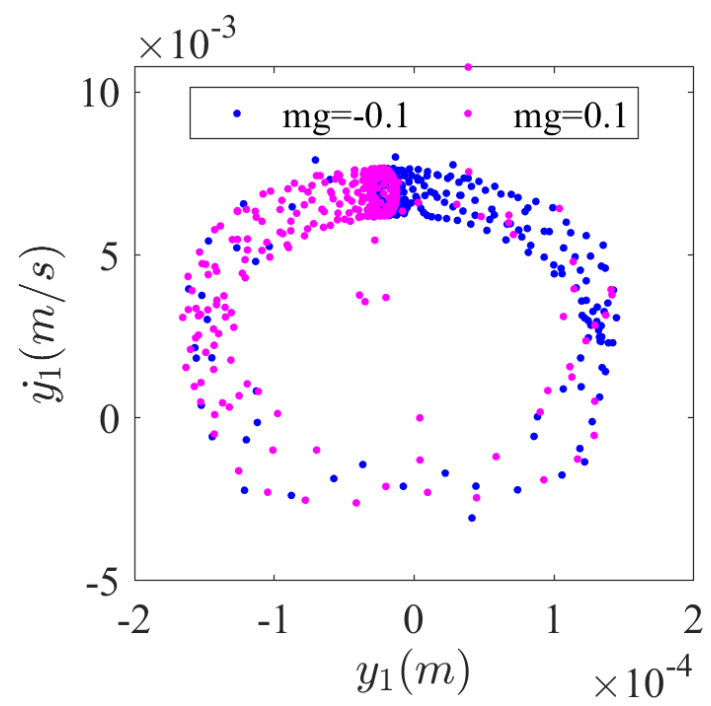

(n) The velocity displacement Poincare section of $y$ direction

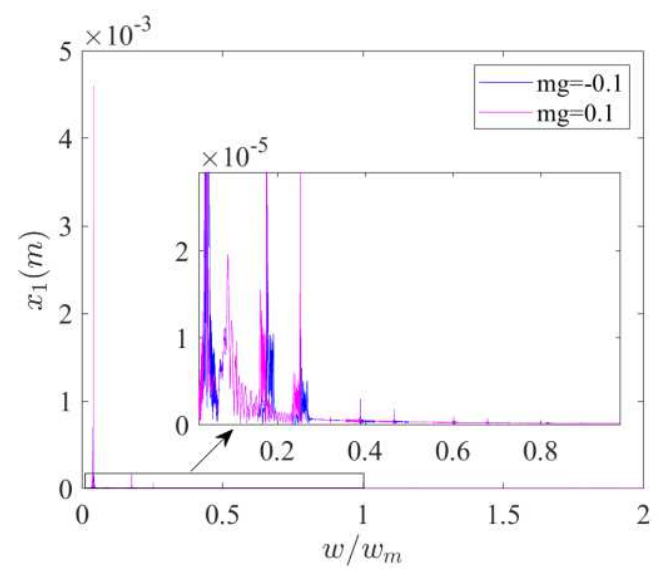

(k) The frequency spectrum of $x$ direction

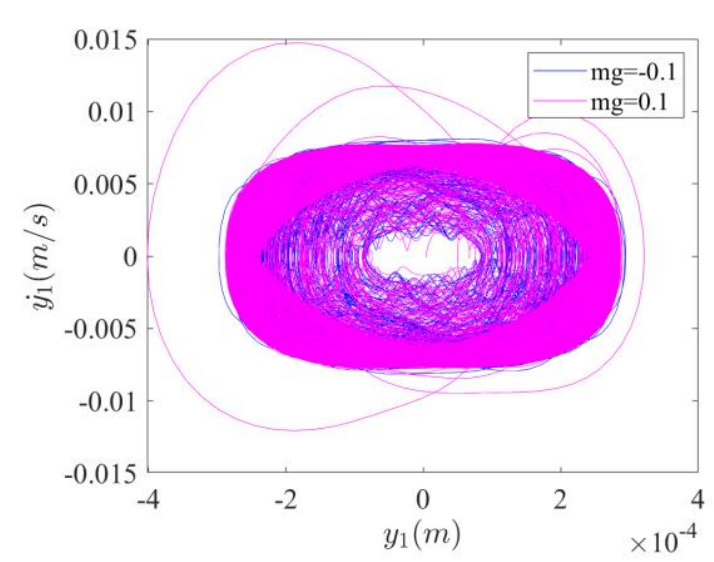

(m) The velocity displacement phase plan of $y$ direction

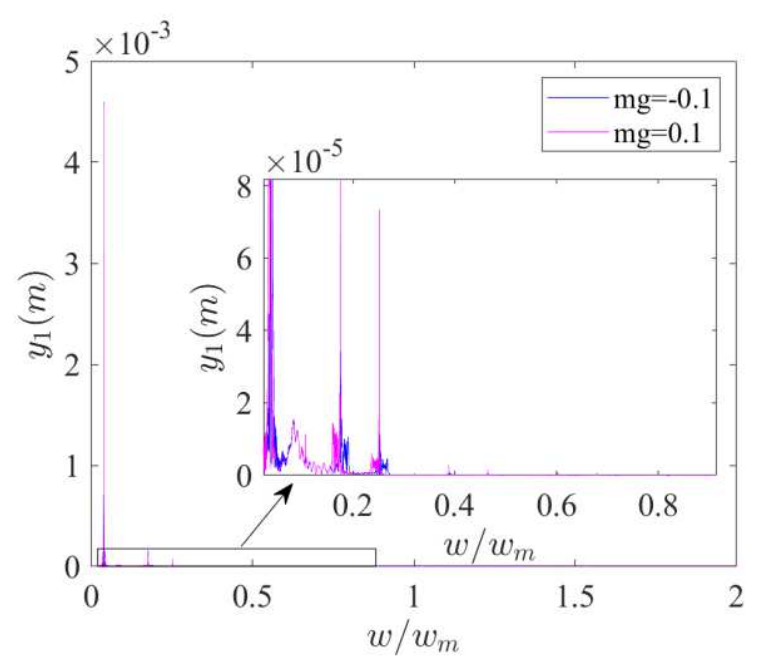

(o) The frequency spectrum of $y$ direction 


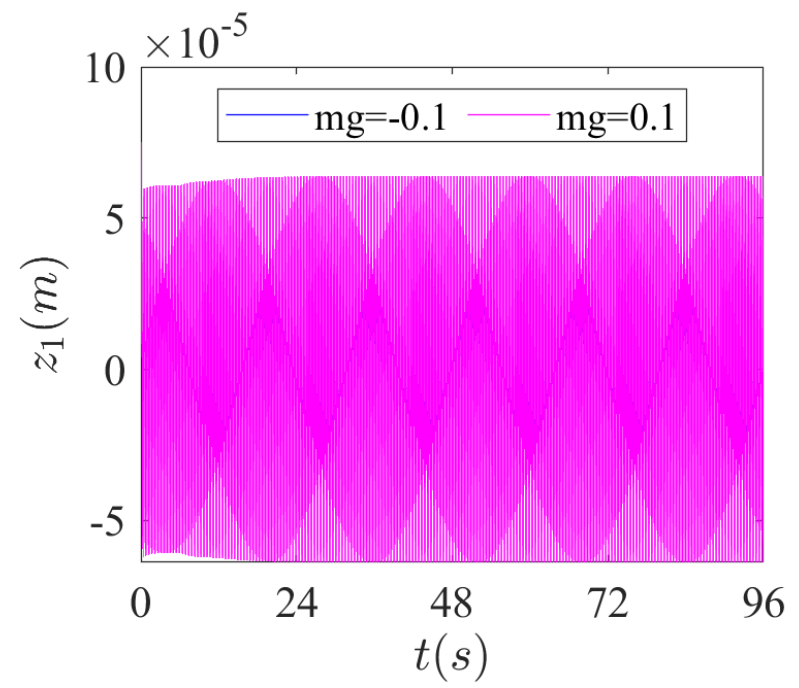

(p)The time-domain diagram of $z$ direction

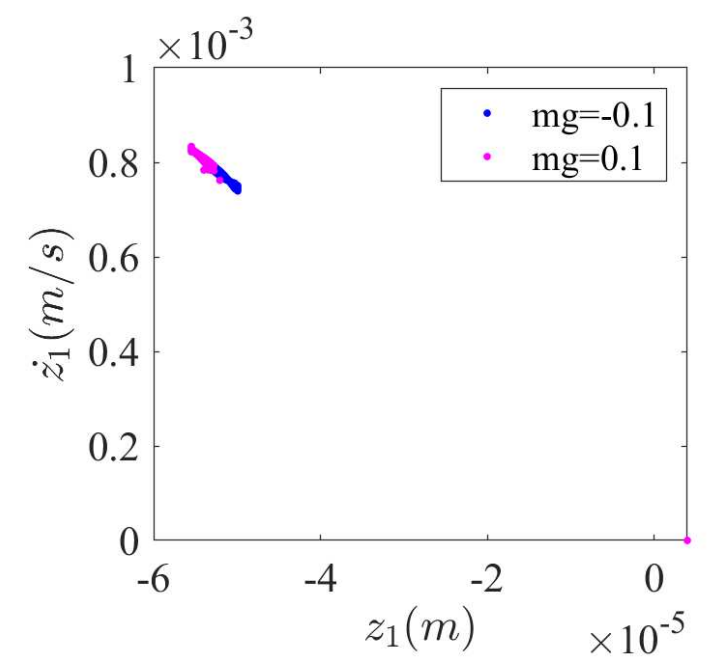

(r) The velocity displacement Poincare section of $z$ direction

Fig. 12 The nonlinear dynamic characteristics of generator vibration

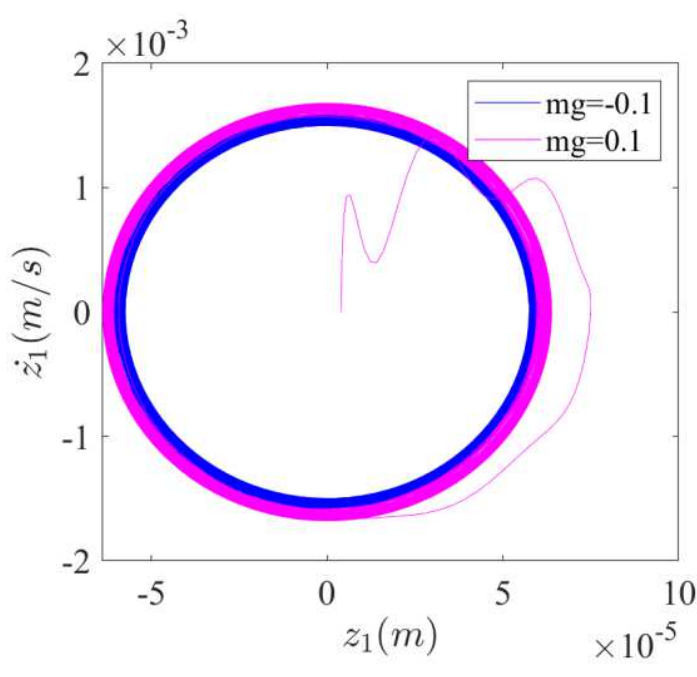

(q) The velocity displacement phase plan of $z$ direction

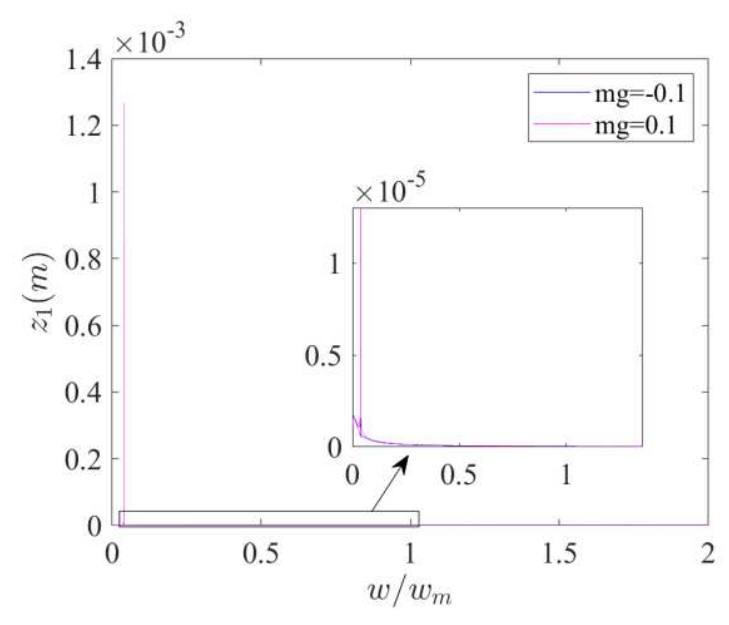

(s) The frequency spectrum of the $z$ direction

(2) The nonlinear dynamic characteristics of hydro-turbine vibration

The hydro-turbine vibration nonlinear dynamic characteristics are shown in Fig.13. From Fig.13(a), the hydro-turbine vibration axis locus is multiple closed curves in $3 \mathrm{D}$ space, which shows quasi-periodic vibration. As can be seen from Fig.13 (b), (c), (d), (e), (f), and (g), the hydro-turbine vibration axis locus are multiple closed curves in $x-y$, $x-z, y-z$ 2D space, and the Poincare section show the attractors are a finite number, which can qualitatively analyze that the hydro-turbine vibration of $x-y, x-z, y-z$ 2D space are quasi-periodic. As can be seen from Fig.13 (h) and (1), during load disturbance, the hydro-turbine vibration of $x$ and $y$ direction go through small fluctuations and 
$y$ direction velocity displacement phase plane are multiple closed curves and the velocity displacement Poincare section show the attractors are a finite number, which can qualitatively analyze that the hydro-turbine vibration of $x$ and $y$ direction are quasi-periodic. As can be seen from Fig.13 (k) and (o), the hydro-turbine vibration of $x$ and $y$ directions vibration is accompanied by three main vibration frequency components, namely, 0.1 octaves, 0.34octaves, 0.56 octaves, 0.78 octaves, and 0.96 octaves. As can be seen from Fig. 13 (p), during load disturbance the hydro-turbine vibration of $z$ direction changes with the corresponding change of load and stable in a certain area, which can be analyzed qualitatively the vibration and the load of the HTGU stay the positive correlation. As can be seen from Fig.13 (q) and (r), the hydro-turbine vibration of $z$ direction velocity displacement phase plan are multiple closed curves, and the velocity displacement Poincare section shows the attractors are a finite number, which can qualitatively analyze that the hydro-turbine vibration of $z$ direction is quasi-periodic. As can be seen from Fig.13 (s), the hydro-turbine vibration of $z$ direction is accompanied by 0.04 octaves.

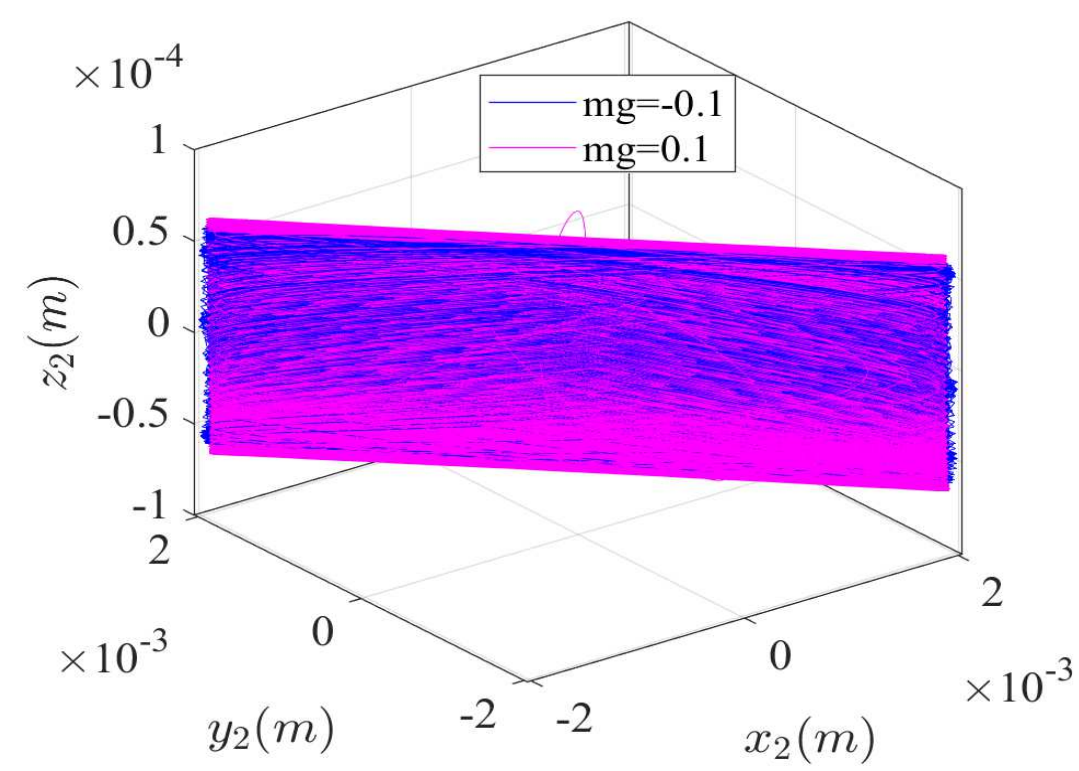

(a) Three-dimensional axis locus of hydro-turbine vibration 


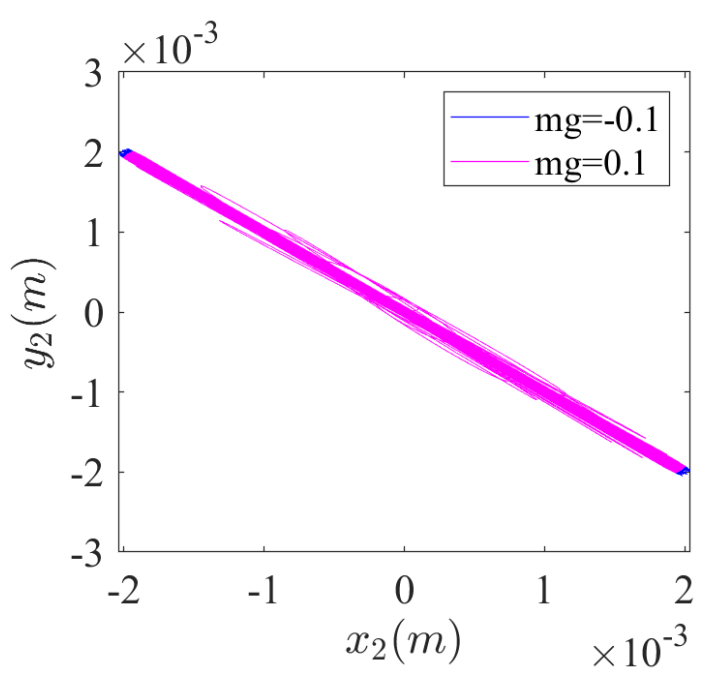

(b) The axis locus of $x-y$ direction

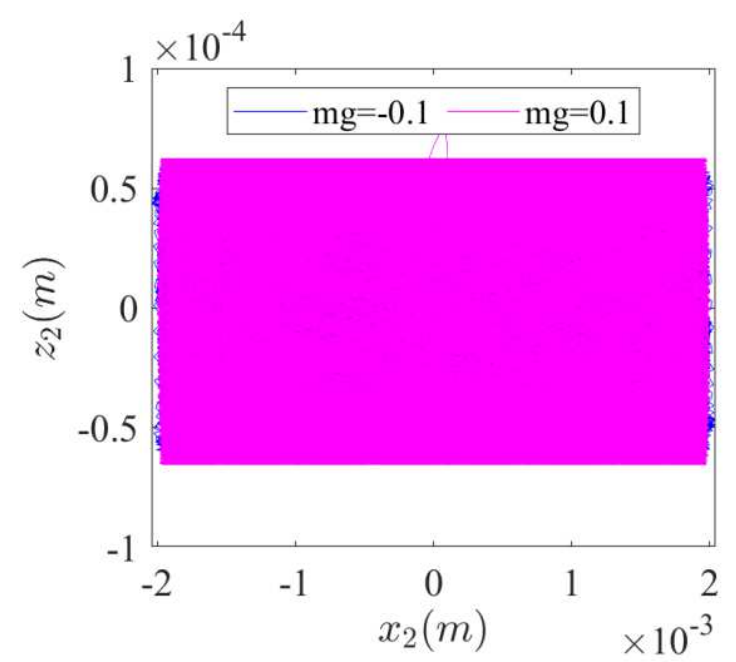

(d) The axis locus of $x-z$ direction

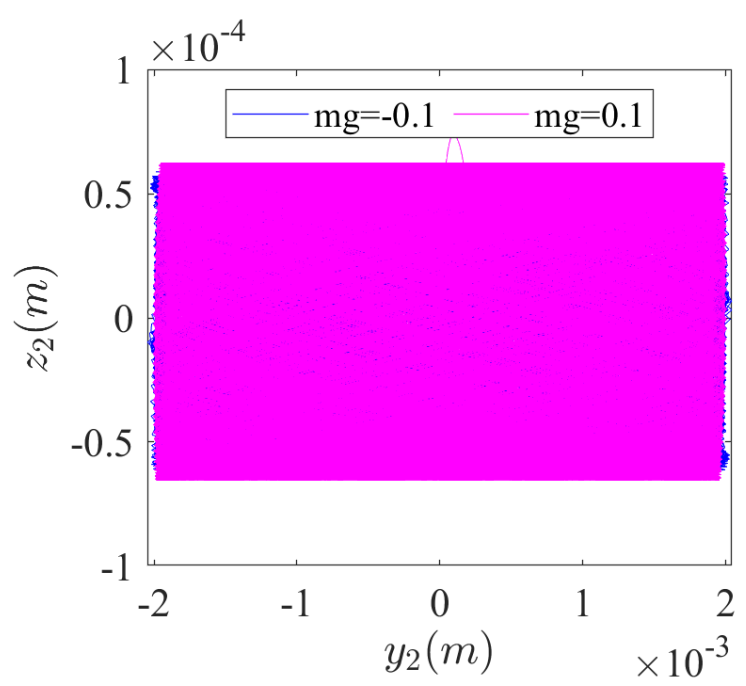

(f) The axis locus of $y-z$ direction

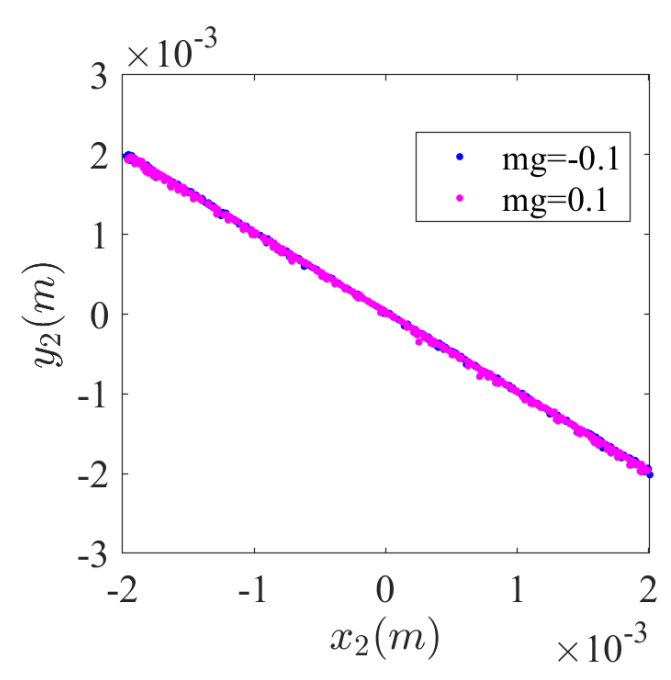

(c)The Poincare section of $x-y$ direction

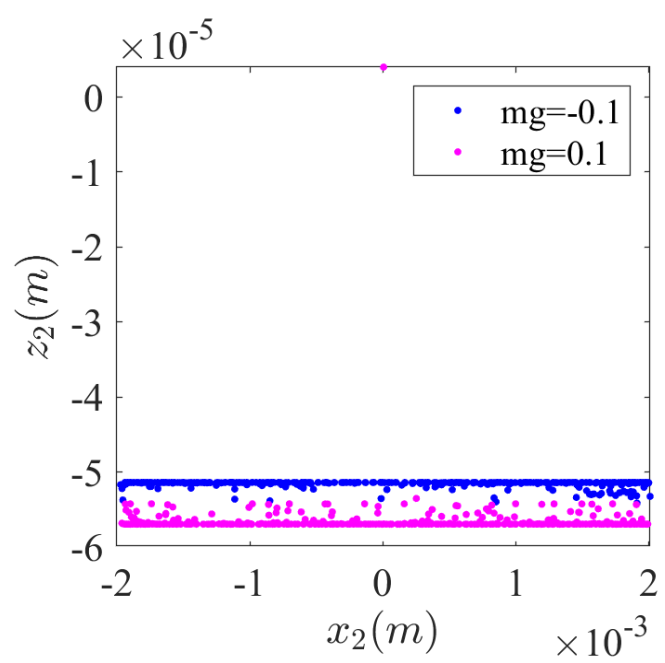

(e)The Poincare section of $x-z$ direction

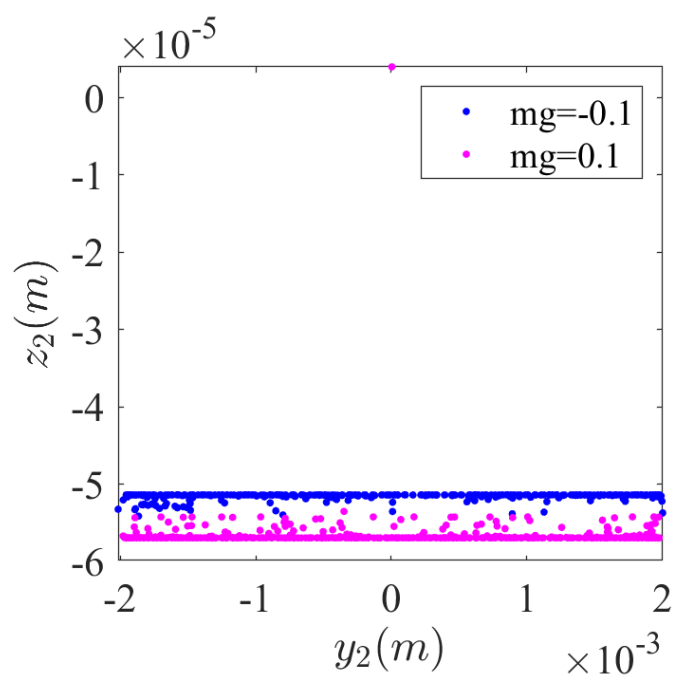

(g)The Poincare section of $y-z$ direction 


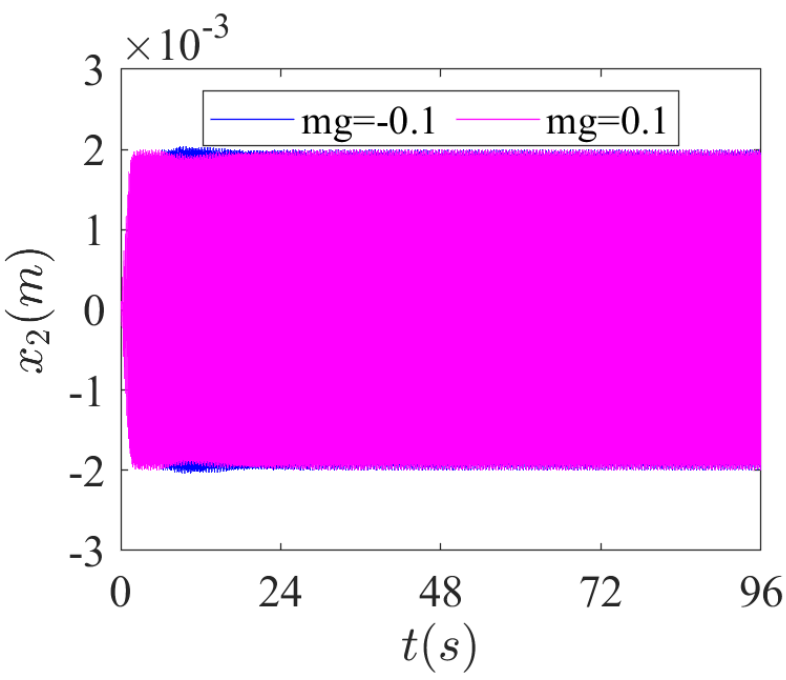

(h) The time-domain diagram of $x$ direction

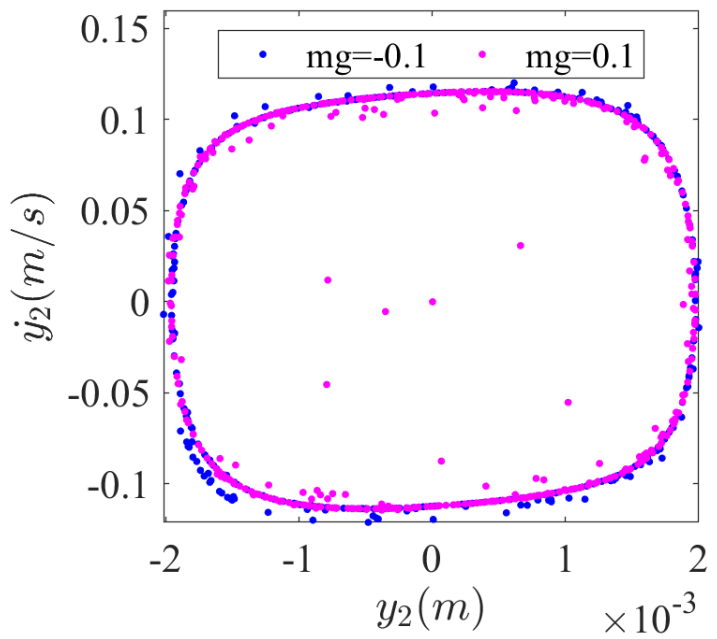

(j) The velocity displacement Poincare section of $x$ direction

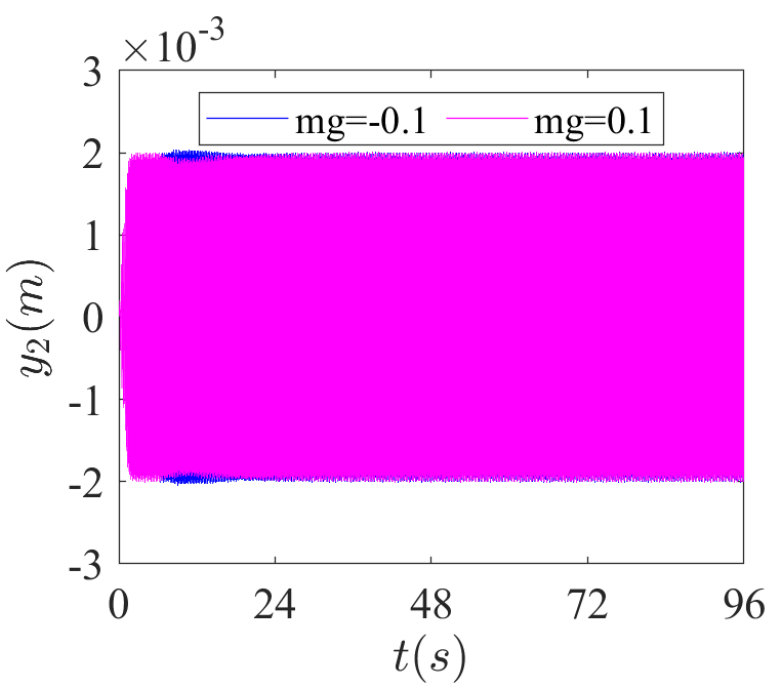

(1) The time-domain diagram of $y$ direction

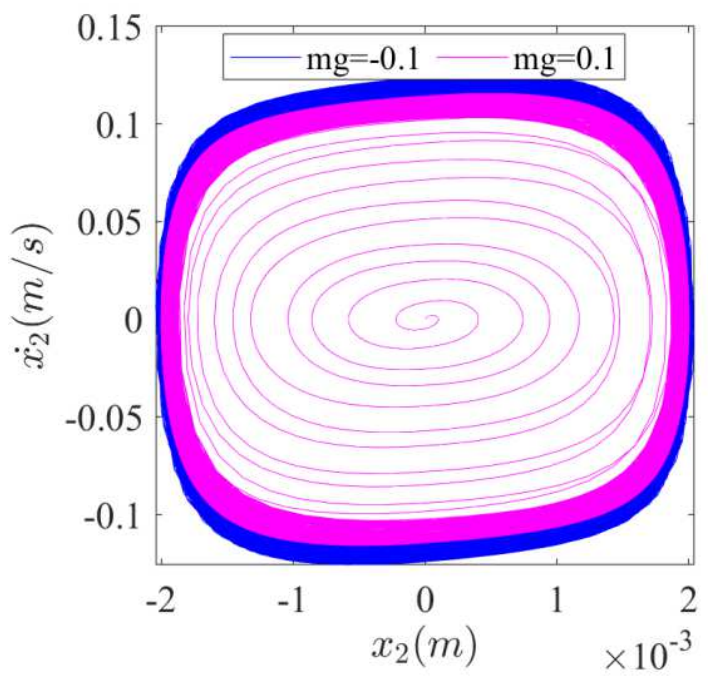

(i) The velocity displacement phase plan of $x$ direction

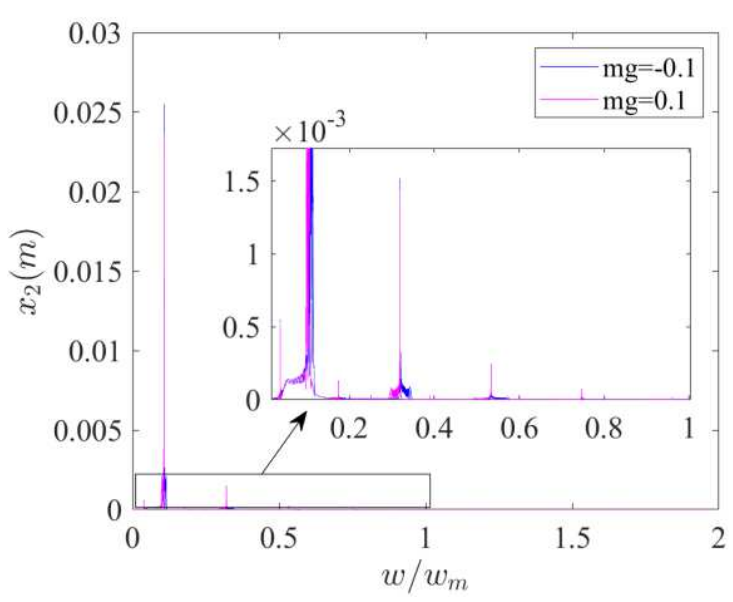

(k) The frequency spectrum of $x$ direction

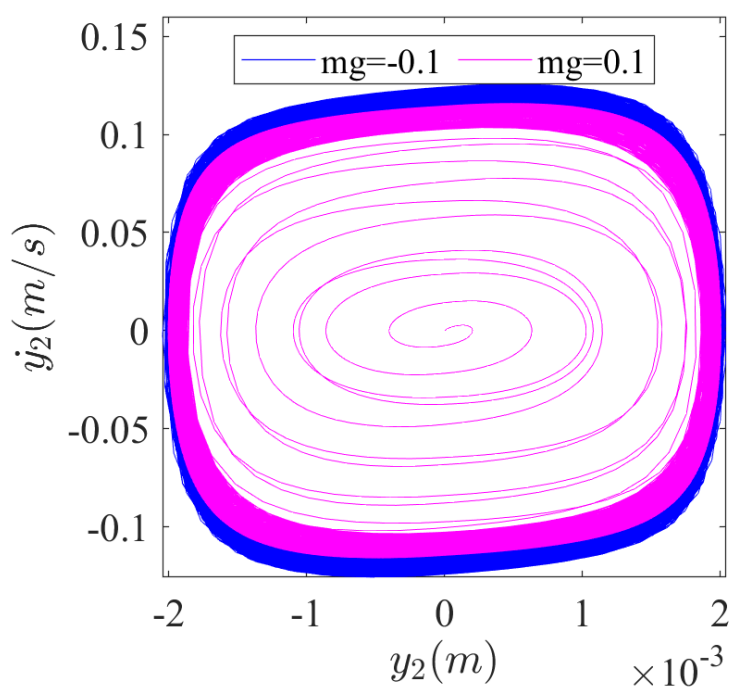

(m) The velocity displacement phase plan of $y$ direction 


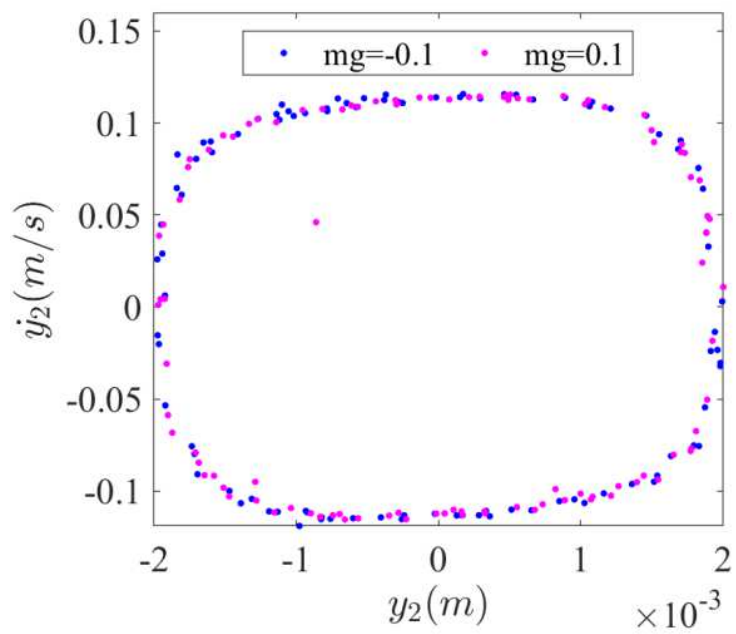

(n) The velocity displacement Poincare section of $y$ direction

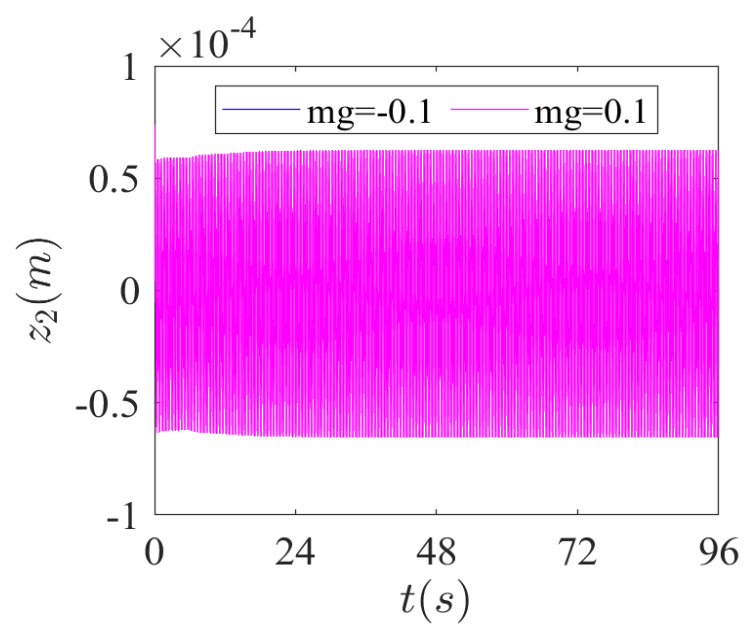

(p) The time-domain diagram of $z$ direction

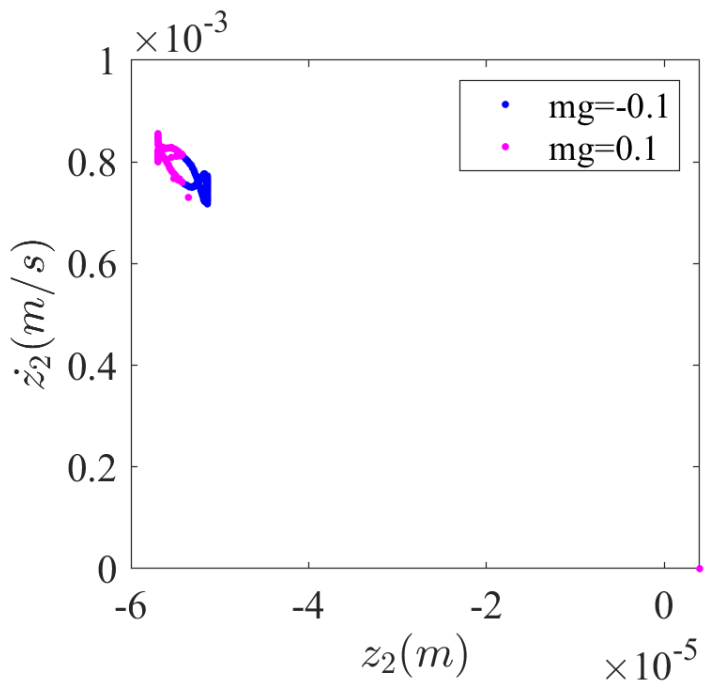

(r) The velocity displacement Poincare section of $z$ direction

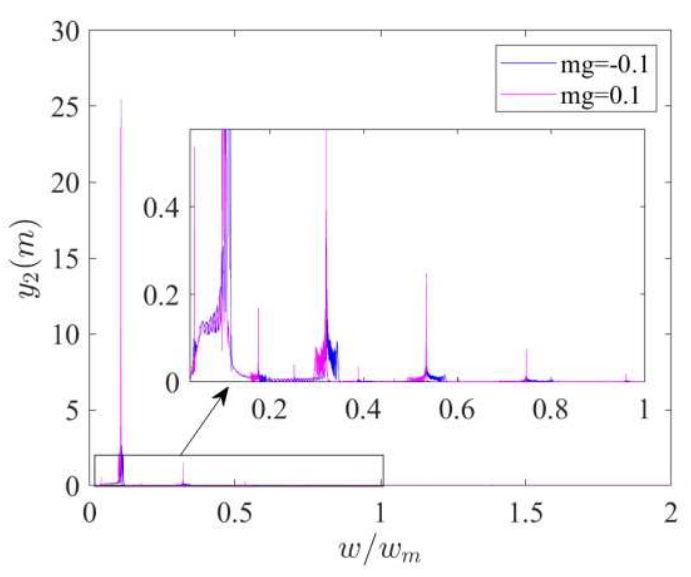

(o) The frequency spectrum of $y$ direction

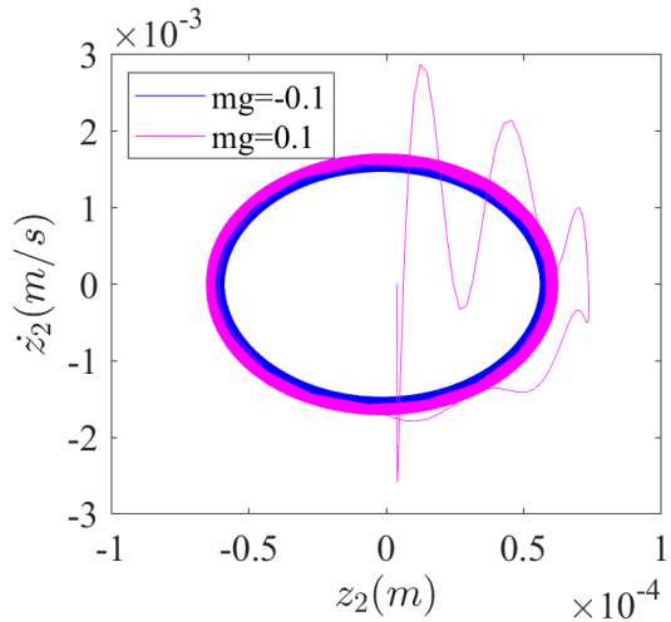

(q) The velocity displacement phase plan of $z$ direction

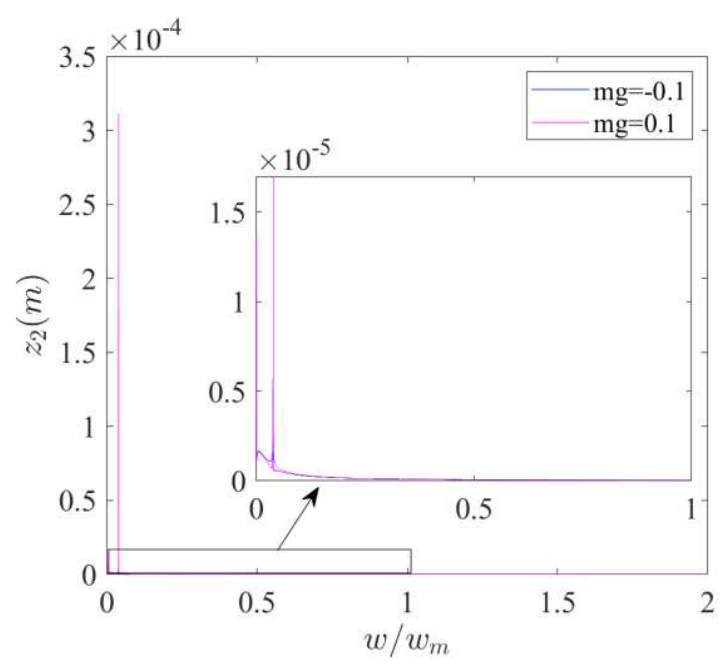

(s) The frequency spectrum of $z$ direction

Fig.13 The nonlinear dynamic characteristics of hydro-turbine vibration

1 (3) The nonlinear dynamic characteristics of torsion vibration 


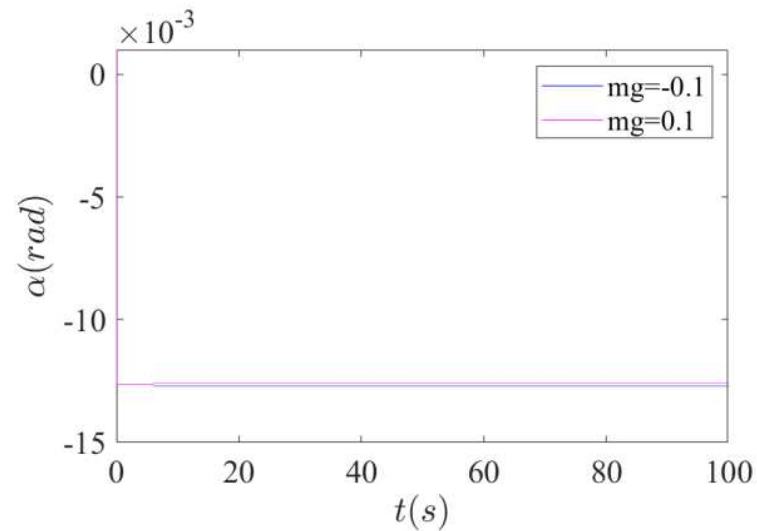

(a) Torsion vibration time-domain diagram

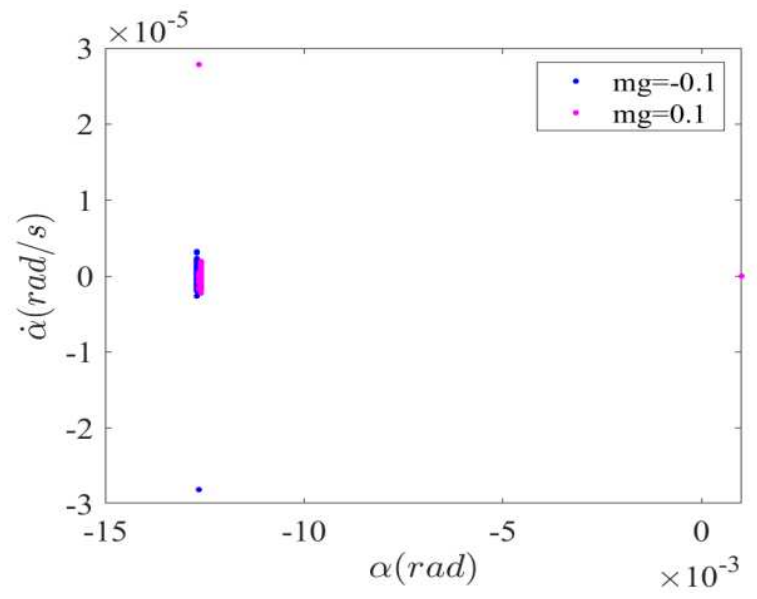

(c) Torsion vibration velocity displacement Poincare section

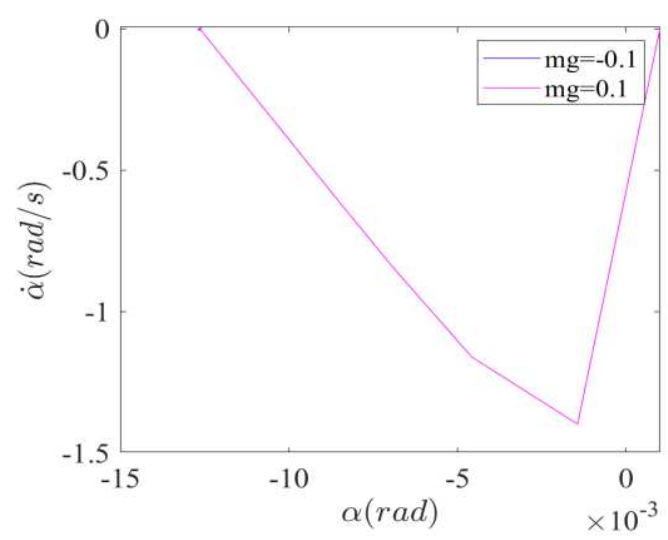

(b) Torsion vibration velocity displacement phase plan

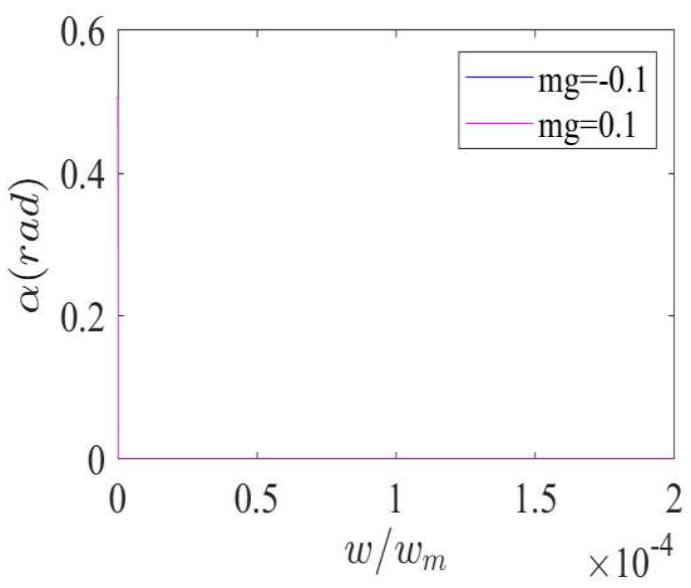

(d) Torsion vibration frequency spectrum

Fig.14 The nonlinear dynamic characteristics of torsion vibration

\section{Conclusions}

In this study, first, we establish a nonlinear mathematical model that considers the HTGS and the shafting system. 
characteristics of the HTGS and the shafting system vibrations which are controlled by the optimized PID parameter were analyzed. The major conclusions are summarized as follows:

(1) The simulation results show that the nonlinear mathematical model coupling the HTGS and the shafting system is effective. It can reveal the interaction characteristics between the shafting system and the HTGS and reveal the stability and sensitivity of some key parameters to the HTGS and shafting system vibrations, and these results will provide some bases for the design and stable operation of the HTGU.

(2) The multi-objective PID parameter optimization strategy shows good performance, it makes the adjusting-time shorten 12 seconds of the HTGS and the shafting system vibrations. The self-regulating coefficient can reduce the overshoot of the HTGS effectively, and suppressed the fluctuation amplitude.

(3) The rotation speed deviation and shafting system vibrations have different stability and sensitivity to the PID parameters. The relative stable region of $k_{p}, k_{i}$, and $k_{d}$ will decrease when considering the shafting system vibrations, the specific region are $k_{p s}=[2.1,3.85], \quad k_{i s}=[0.26,0.59]$, and $k_{d s}=[0.1,5.4]$, respectively.

(4) The generator and hydro-turbine vibrations in the 3D space that excited by the mass eccentricity, the UMP, the arcuate whirl of the rotor, and the seal excitation coupled vibration sources are quasi-periodic composed of several frequency components, whereas the torsion vibration is stable with a tiny frequency component,

\section{acknowledgment}

This work was supported by the National Key R\&D Program of China (GrantNo.2016YFC0402205), National Natural Science Foundation of China(U185202), National Natural Science Foundation of China(52039004)

\section{Declaration of interests}

The authors declare that they have no known competing financial interests or personal relationships that could have appeared to influence the work reported in this paper.

\section{References:}

[1] Zhu D., Tao R., Xiao R, et al. Solving the runner blade crack problem for a Francis hydro-turbine operating 
under condition-complexity[J]. Renewable Energy, 2020, 149:298-320.

[2] Wang CS, Yan JY, Marnay C, Djilali N, Dahlquist E, Wu JZ, et al. Distributed Energy and Microgrids (DEM). Appl Energy 2018;210:685-9.

[3] Z. Ma, B. Zhu, C. Rao, et al., Comprehensive hydraulic improvement and parametric analysis of a Francis turbine runner, Energies 12 (2) (2019) 307.

[4] IEEE Group. Hydraulic-turbine and turbine control-models for system dynamic studies. IEEE Trans Power Systems 1992;7(1):167-79.

[5] Xu BB, Chen DY, Zhang H, Zhou R. Dynamic analysis and modeling of a novel fractional-order hydro-turbine-generator unit. Nonlinear Dyn 2015;81(3):1263-74.

[6] Dong Liu, Xin Wang, Yunshui Peng, Hui Zhang, Zhihuai Xiao, Xiangdong Han, O.P. Malik, Stability analysis of hydropower units under full operating conditions considering turbine nonlinearity, Renewable Energy, Volume 154, 2020, Pages 723-742.

[7] M.H. Chaudhry, Transients in Hydroelectric Power Plants, Springer, New York, NY, 2014.

[8] S. Hagihara, H. Yokota, K. Goda, K. Isobe, Stability OF a hydraulic-turbine generating-unit controlled BY PID governor, IEEE Trans. Power Apparatus Syst. 98 (6) (1979) 2294-2298.

[9] X.D. Yu, J. Zhang, C.Y. Fan, S. Chen, Stability analysis of governor-turbine hydraulic system by state space method and graph theory, Energy 114 (2016) 613-622.

[10] H.W. Luo, J.G. Zhang, W.J. Du, J.R. Lu, X.L. An, Nonlinear dynamics of a PI hydro-turbine governing system with double delays, J. Contr. Sci. Eng. (2017)14.

[11] D.J. Ling, Y. Tao, An analysis of the Hopf bifurcation in a hydro-turbine governing system with saturation, IEEE Trans. Energy Convers. 21 (2) (2006)512-515.

[12] X.J. Lai, C.S. Li, W.C. Guo, Y.H. Xu, Y.G. Li, Stability and dynamic characteristics of the nonlinear coupling system of hydropower station and power grid, Commun. Nonlinear Sci. Numer. Simulat. 79 (2019) 20.

[13] W.C. Guo, J.D. Yang, M.J. Wang, X. Lai, Nonlinear modeling and stability analysis of hydro-turbine governing system with sloping ceiling tailrace tunnel under load disturbance, Energy Convers. Manag. 106 (2015) $127-138$.

[14] F.F. Wang, D.Y. Chen, B.B. Xu, H. Zhang, Nonlinear dynamics of a novel fractional-order Francis hydro-turbine governing system with time delay, Chaos, Solit. Fractals 91 (2016) 329-338.

[15] Liang J., Yuan X., Yuan Y., et al. Nonlinear dynamic analysis and robust controller design for Francis hydraulic turbine regulating system with a straight-tube surge tank[J]. Mechanical Systems and Signal Processing, 2017, 85:927-946.

[16] Qu F., Guo W . Robust Ho control for hydro-turbine governing system of hydropower plant with super long headrace tunnel[J]. International Journal of Electrical Power \& Energy Systems, 2021, 124:106336.

[17] Chen Z., Yuan X., Ji B., et al. Design of a fractional order PID controller for hydraulic turbine regulating system using chaotic non-dominated sorting genetic algorithm II[J]. Energy Conversion and Management, 2014, 84:390-404.

[18] Wu X., Xu Y., Liu J., et al. Characteristics Analysis and Fuzzy Fractional-Order PID Parameter Optimization for Primary Frequency Modulation of a Pumped Storage Unit Based on a Multi-Objective Gravitational Search Algorithm[J]. Energies, 2019, 13.

[19] Zhang, L., Z. Ma and B. Song, Dynamic characteristics of a rub-impact rotor-bearing system for hydraulic generating set under unbalanced magnetic pull. Archive of Applied Mechanics, 2013. 83(6): p. 817-830.

[20] Fu, X.Q., et al., Imbalance-misalignment-rubbing coupling faults in hydraulic turbine vibration. Optik, 2016. 127(8): p. 3708-3712.

[21] Song, Z., et al., Torsional Vibration Analysis of Hydro-Generator Set Considered Electromagnetic and Hydraulic Vibration Resources Coupling. International Journal of Precision Engineering and Manufacturing, 2018. 19(7): p. 939-945. 
[22] Zhong, W., et al., Integrated Shock Absorber With Both Tunable Inertance and Damping. Frontiers in Materials, 2020. 7.

[23] An, X., et al., Dynamic response of a rub-impact rotor system under axial thrust. Archive of Applied Mechanics, 2009. 79(11): p. 1009-1018.

[24] Zhuang, K., et al., Dynamic Analyses of the Hydro-Turbine Generator Shafting System Considering the Hydraulic Instability. Energies, 2018. 11(10): p. 2862.

[25] Gao, Y., et al., A hybrid of FEM simulations and generative adversarial networks to classify faults in rotor-bearing systems. ISA Transactions, 2020.

[26] Li, J., et al., Analysis of the gyroscopic effect on the hydro-turbine generator unit. Mechanical Systems and Signal Processing, 2019. 132: p. 138-152.

[27] $\mathrm{Xu}, \mathrm{B}$., et al., Shaft mis-alignment induced vibration of a hydraulic turbine generating system considering parametric uncertainties. Journal of Sound and Vibration, 2018. 435: p. 74-90.

[28] Zhang, L., et al., Transient vibration analysis of unit-plant structure for hydropower station in sudden load increasing process. Mechanical Systems and Signal Processing, 2019. 120: p. 486-504.

[29] Zhang, H., et al., Nonlinear modeling and dynamic analysis of hydro-turbine governing system in the process of load rejection transient. Energy Conversion and Management, 2015. 90: p. 128-137.

[30] Xu, B., et al., Sensitivity analysis of a Pelton hydropower station based on a novel approach of turbine torque. Energy Conversion and Management, 2017. 148: p. 785-800.

[31] $\mathrm{Xu}, \mathrm{B}$., et al., Hamiltonian modeling of multi-hydro-turbine governing systems with sharing common penstock and dynamic analyses under shock load. Energy Conversion and Management, 2016. 108: p. 478-487.

[32] Zeng, Y., et al., The generalized Hamiltonian model for the shafting transient analysis of the hydro turbine generating sets. Nonlinear Dynamics, 2014. 76(4): p. 1921-1933.

[33] Li, H., et al., Pumping phase modulation analysis for operational quality of a pumped-storage generating system. Energy Conversion and Management, 2019. 199: p. 111989.

[34] $\mathrm{Wu}, \mathrm{Q}$., L. Zhang and Z. Ma, A model establishment and numerical simulation of dynamic coupled hydraulic-mechanical-electric-structural system for hydropower station. Nonlinear Dynamics, 2017. 87(1): p. 459-474.

[35] Sun, W. and D. Yan, Identification of the nonlinear vibration characteristics in hydropower house using transfer entropy. Nonlinear Dynamics, 2014. 75(4): p. 673-691.

[36] Guo, W. and J. Yang, Hopf bifurcation control of hydro-turbine governing system with sloping ceiling tailrace tunnel using nonlinear state feedback. Chaos, Solitons \& Fractals, 2017. 104: p. 426-434.

[37] Childs D W . Dynamic Analysis of Turbulent Annular Seals Based On Hirs' Lubrication Equation[J]. Journal of Lubrication Technology, 1982, 105(3).

[38] Shi Y., Zhou J., Lai X., et al. Stability and sensitivity analysis of the bending-torsional coupled vibration with the arcuate whirl of hydro-turbine generator unit[J]. Mechanical Systems and Signal Processing, 2021.149.

[39] Xi, Zhao, Li, et al. Study on Improving the Empirical Formula of Calculating Francis Turbine's Axial Hydro-thrust[C] 2014 International Conference on Energy Science and Applied Technology(ESAT 2014). 0.

[40] STEIN T. Frequency control under isolated network conditions. Water power. 1970.22(9):320-324.

[41] K. Deb, A. Pratap, S. Agarwal and T. Meyarivan, "A fast and elitist multiobjective genetic algorithm: NSGA-II," in IEEE Transactions on Evolutionary Computation, vol. 6, no. 2, pp. 182-197, April 2002.

[42] Rahmati S H A., Ahmadi A., Karimi B. . Multi-objective evolutionary simulation based optimization mechanism for a novel stochastic reliability centered maintenance problem[J]. Swarm \& Evolutionary Computation, 2018. 
Figures

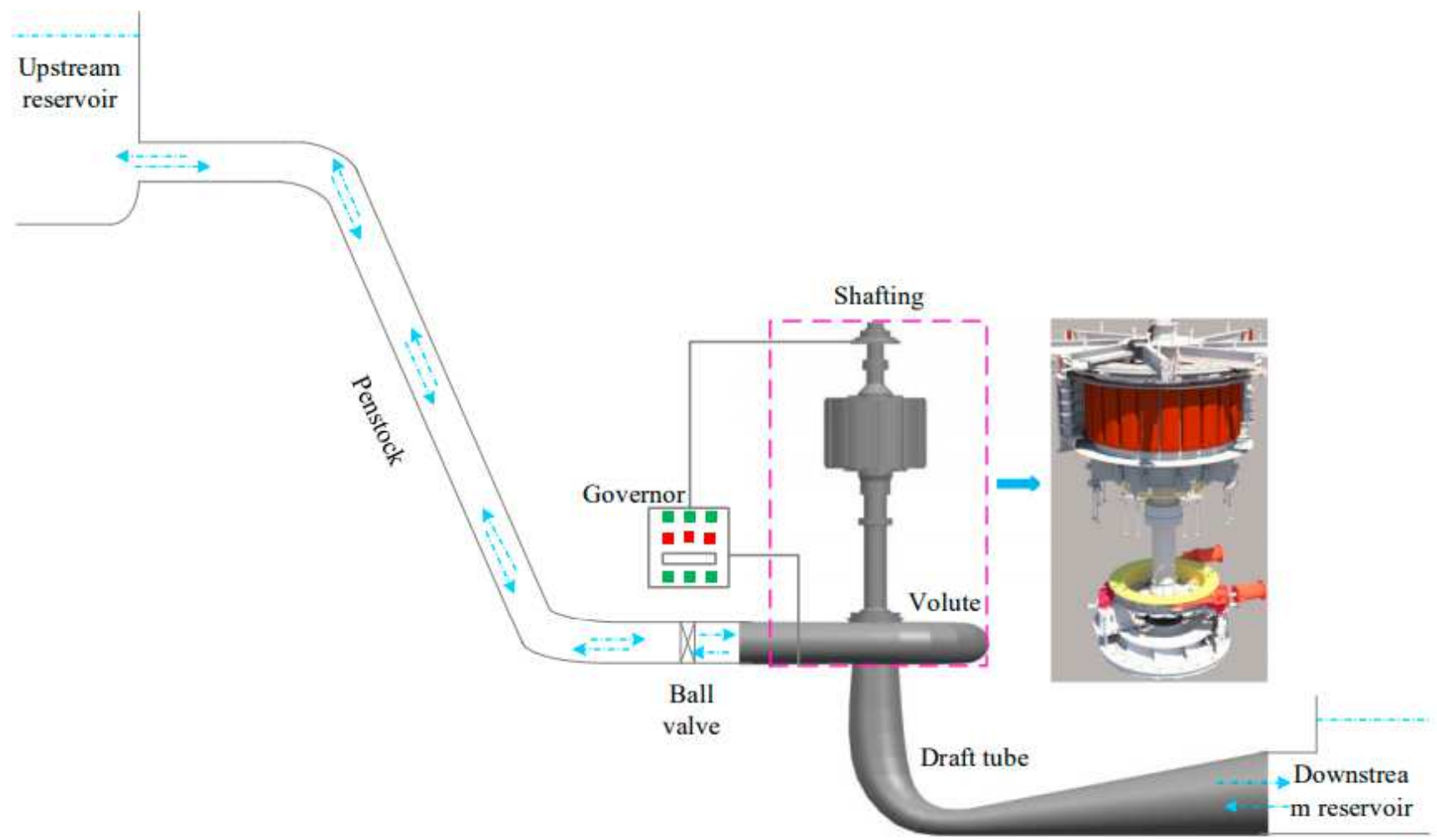

Figure 1

Structure of a hydropower station 


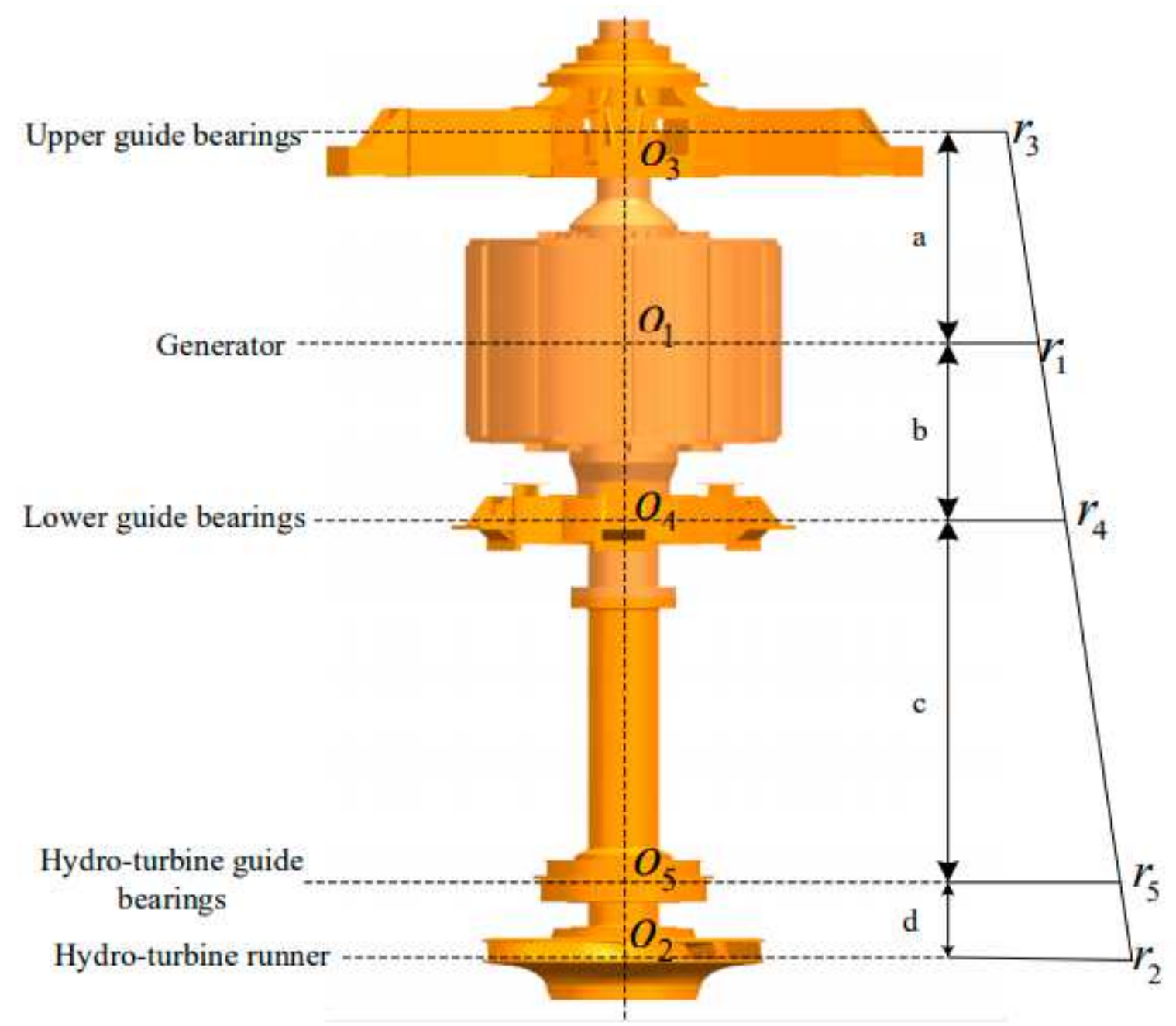

Figure 2

Shafting structure diagram

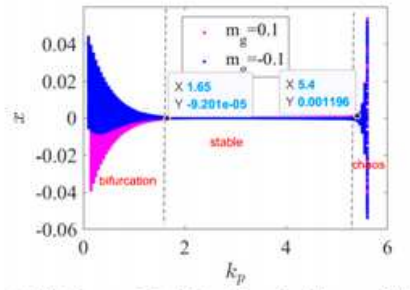

(a) Rotation speed deviation bifurcation diagram of $k$,

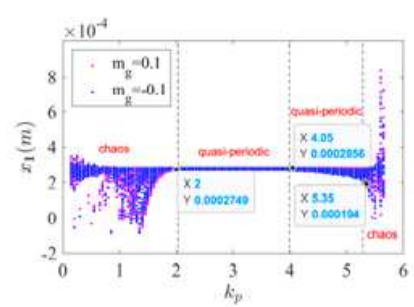

(c) Generator vibration $\left(x_{1}\right)$ bifurcation diagram of $k_{p}$

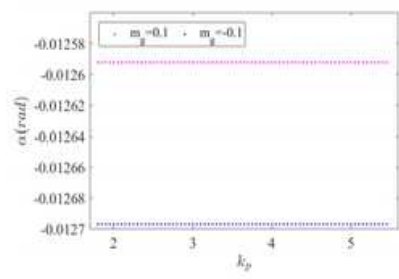

(b) Torsion angle bifurcation diagram of $k$,

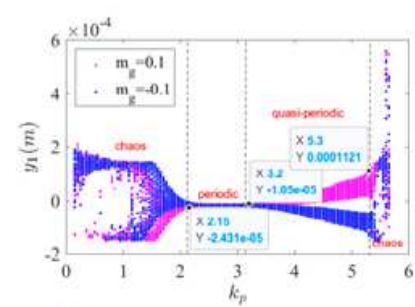

(d) Generator vibration $\left(y_{1}\right)$ bifurcation diagram of $k_{p}$

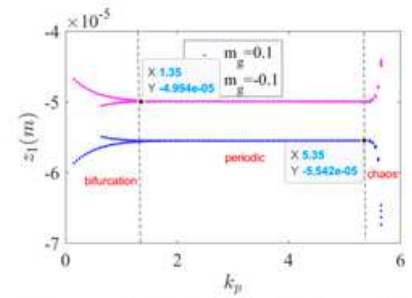

(c) Gencrator vibration $\left(z_{1}\right)$ bifurcation diagram of $k$

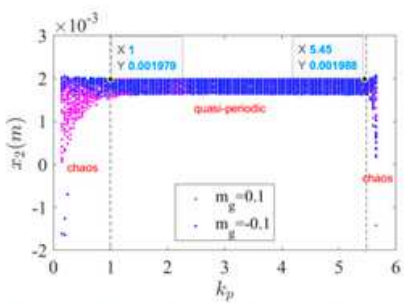

(f) Hydro-turbine vibration $\left(x_{2}\right)$ bifurcation diagram of $k$
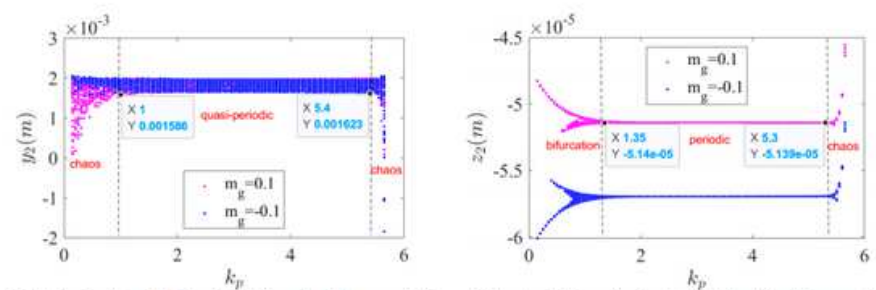

(g) Hydro-turbine vibration $\left(y_{2}\right)$ bifurcation diagram of $k_{p} \quad$ (h) Hydro-turbine vibration $\left(z_{2}\right)$ bifurcation diagram of $k$,

Figure 3 
The rotation speed deviation and SCV dynamic characteristics to Kp

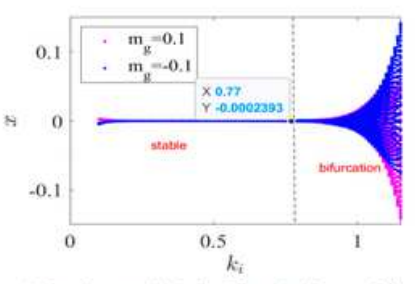

(a) Rotation specd deviation bifurcation diagram of $k$

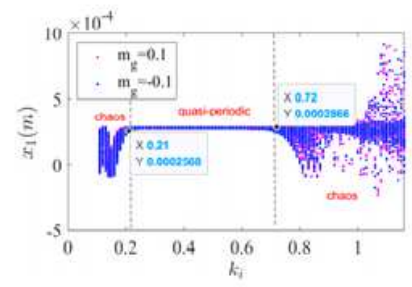

(c) Generator vibration ( $\left.x_{1}\right)$ bifurcation diagram of $k$

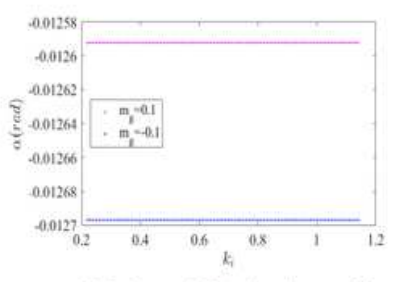

(b) Torsion angle bifurcation diagram of $k$,

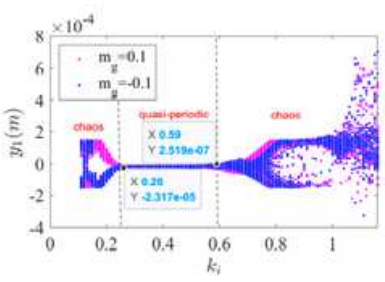

(d) Generator vibration $\left(y_{1}\right)$ bifurcation diagram of $k$

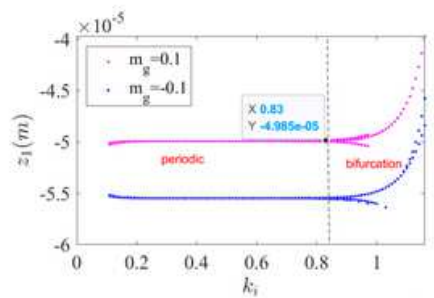

(c) Generator vibration ( $\left.z_{i}\right)$ bifurcation diagram of $k$

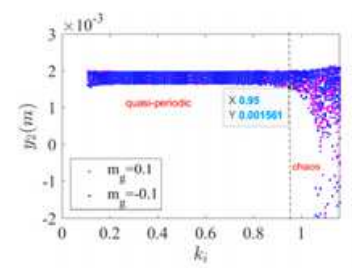

(g) Hydro-turbine vibration $\left(y_{z}\right)$ bifurcation diagram of $k$

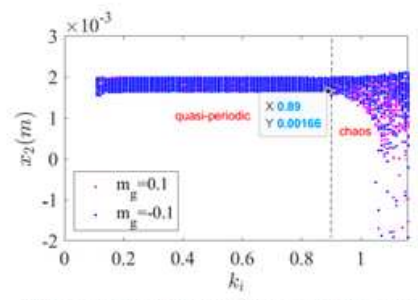

(f) Hydro-turbine vibration $\left(x_{2}\right)$ bifurcation diagram of $k$

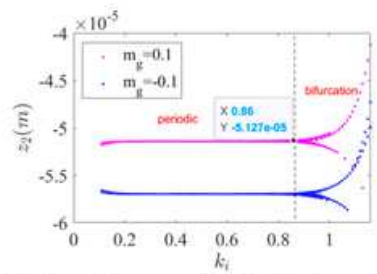

(h) Hydro-turbine vibration $\left(z_{2}\right)$ bifurcation diagram of $k$

\section{Figure 4}

The rotation speed deviation and SCV dynamic characteristics of KI
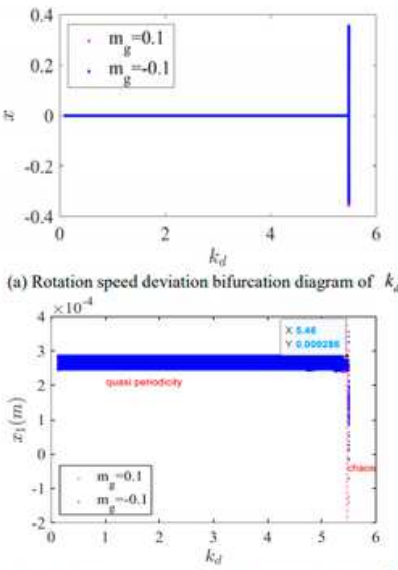

(c) Generator vibration $\left(x_{1}\right)$ bifurcation diagram of $k$
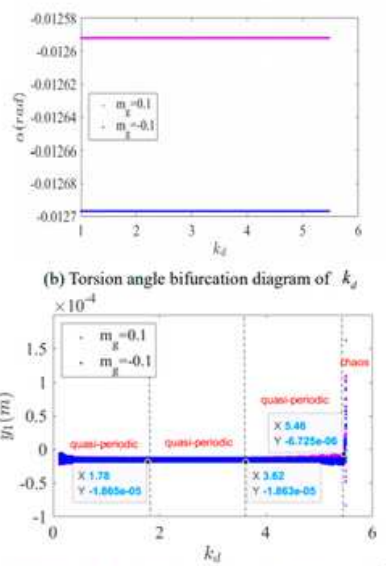

(d) Gencrator vibration $\left(y_{1}\right)$ bifurcation diagram of $k$

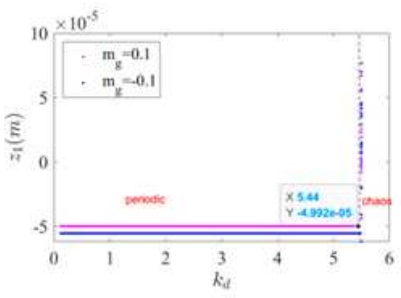

(c) Generator vibration $\left(z_{i}\right)$ bifurcation diagram of $k$

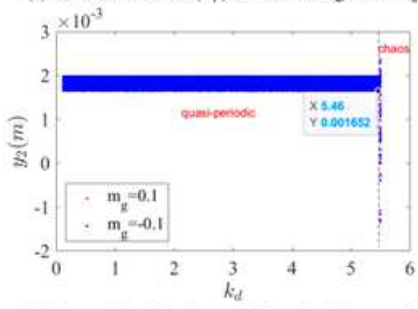

(g) Hydro-turbine vibration $\left(y_{2}\right)$ bifurcation diagram of $k$

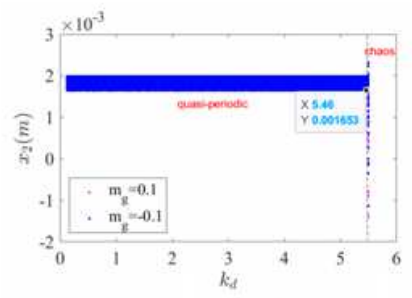

(f) Hydro-turbine vibration $\left(x_{2}\right)$ bifurcation diagram of $k_{d}$

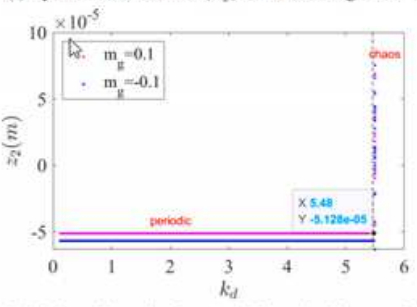

(h) Hydro-turbine vibration $\left(z_{2}\right)$ bifurcation diagram of $k$,

\section{Figure 5}

The rotation speed deviation and SCV dynamic characteristics to $\mathrm{Kd}$ 


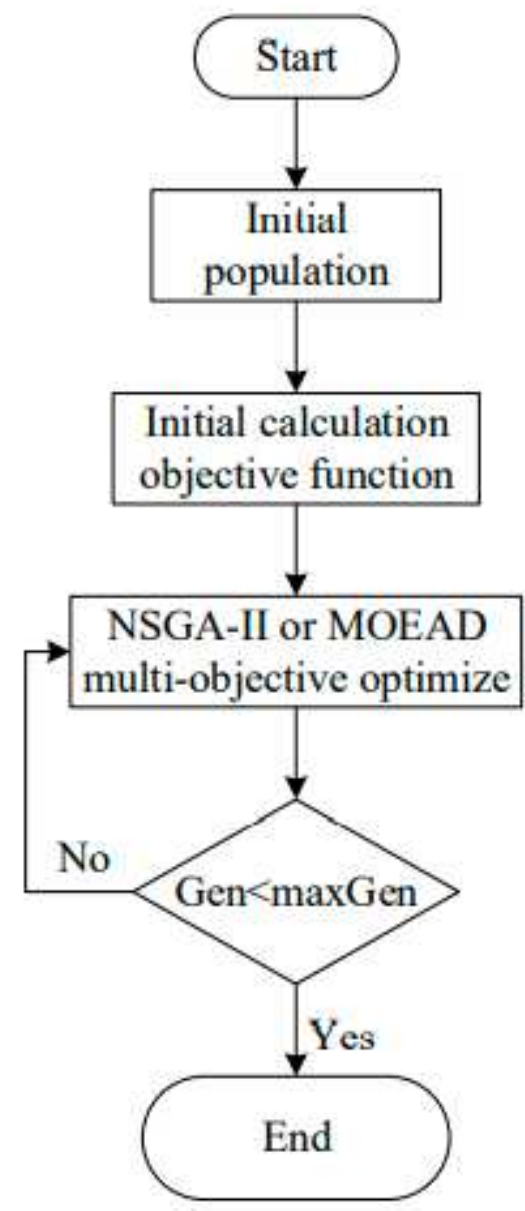

Figure 6

Optimization process diagram

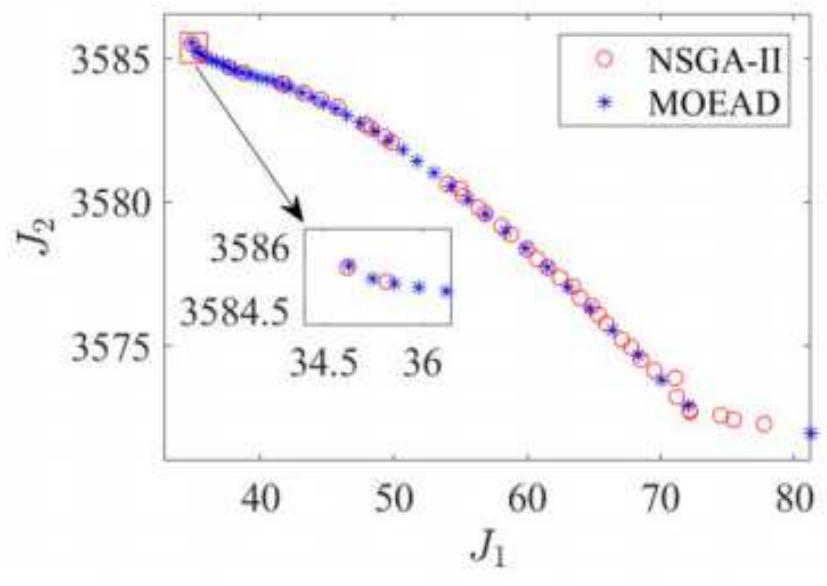

(a) $\mathrm{Mg}=-0.1$

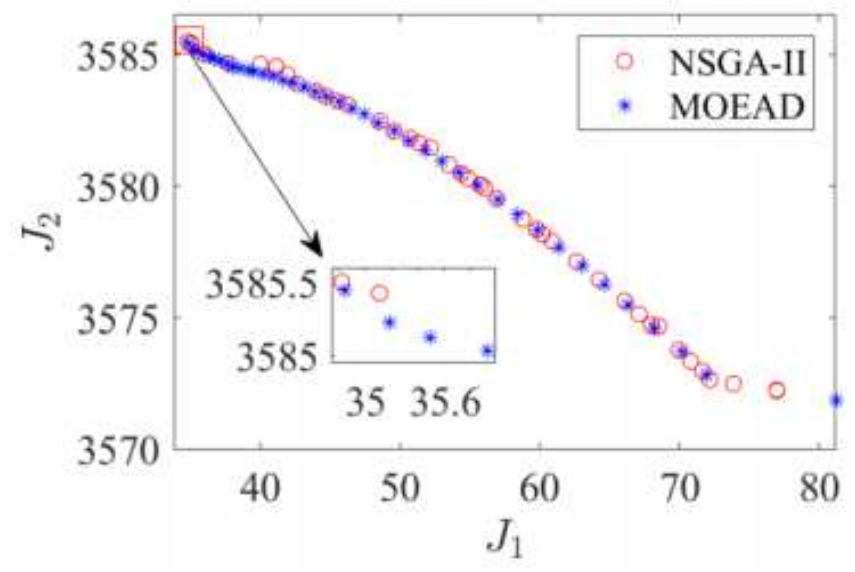

(b) $\mathrm{Mg}=0.1$

Figure 7

Pareto optimal solution obtained by different algorithms 

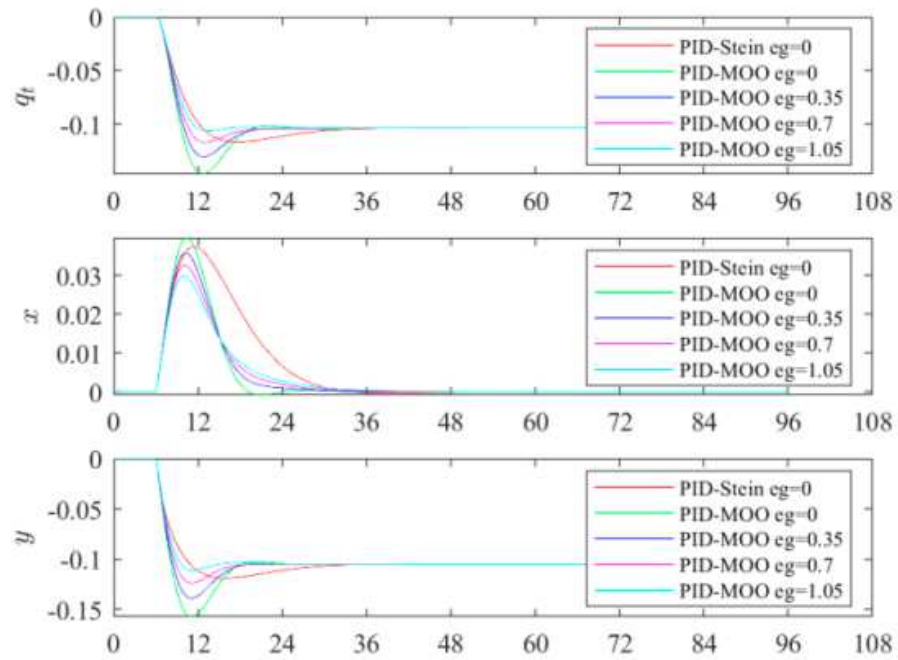

(a) $m_{g}=-0.1$
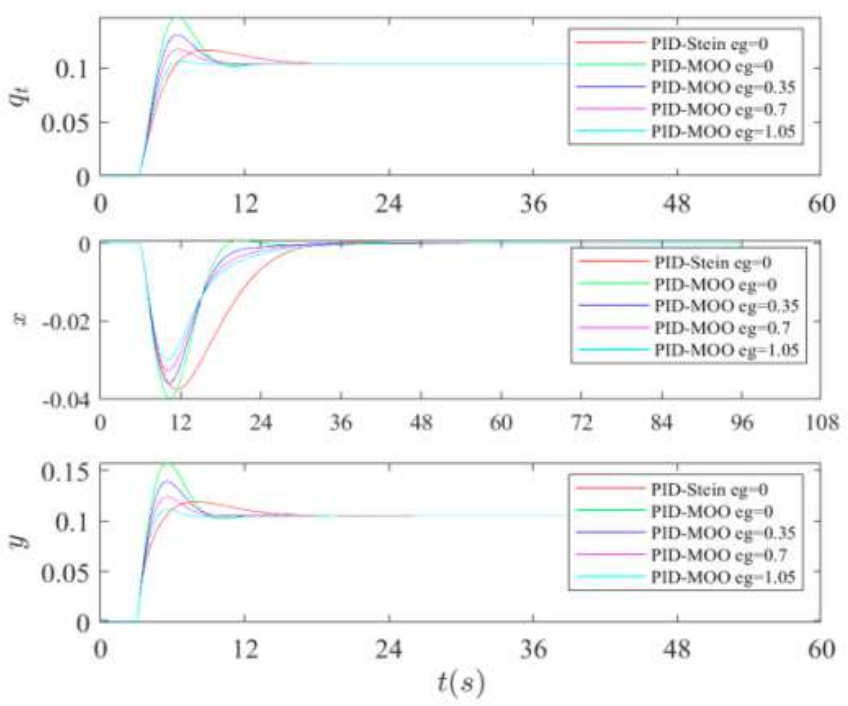

(b) $m_{g}=0.1$

\section{Figure 8}

The nonlinear dynamic characteristics of the HTGS

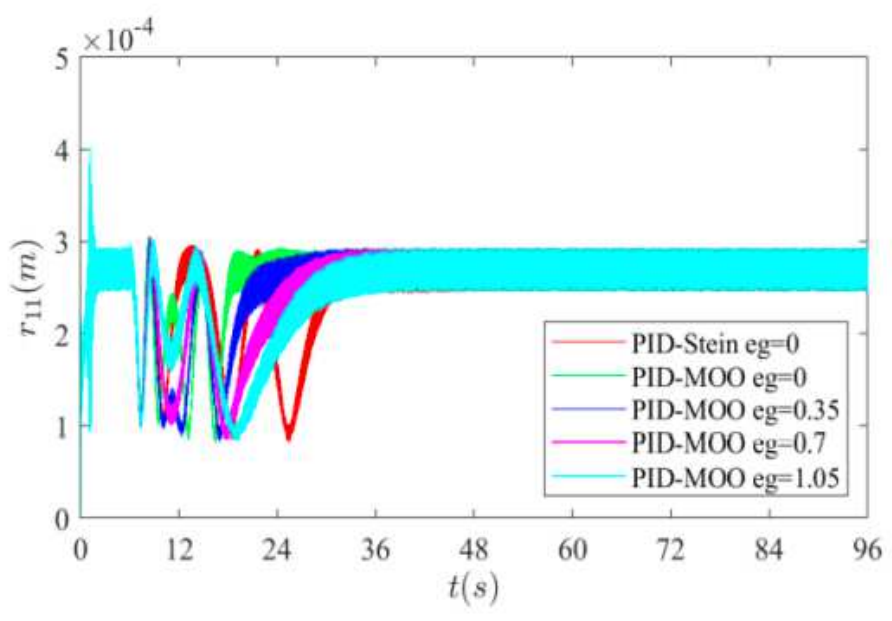

(a) $m_{g}=-0.1$

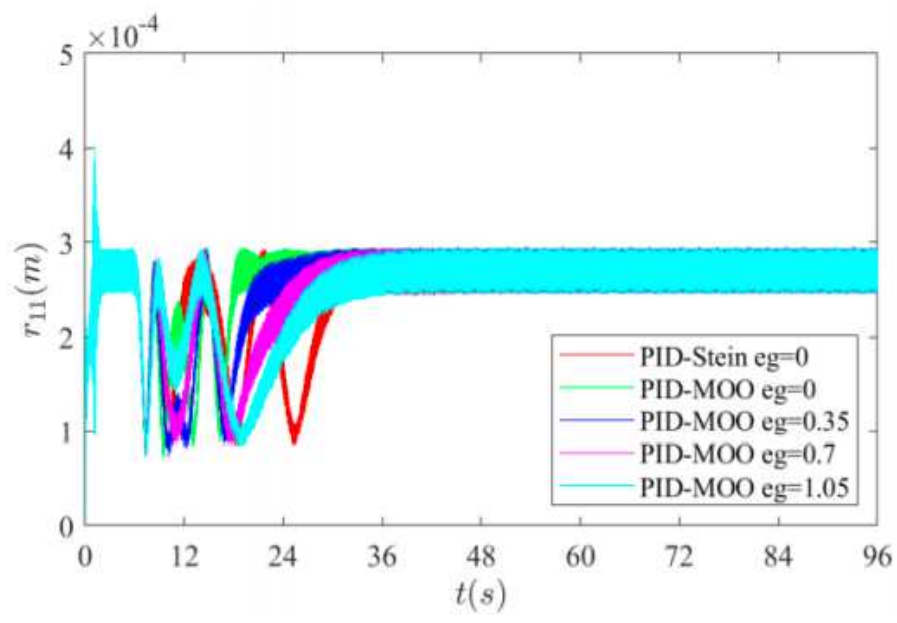

(b) $m_{g}=0.1$

\section{Figure 9}

Robustness of optimized PID controller for to the load-self regulation coefficient 


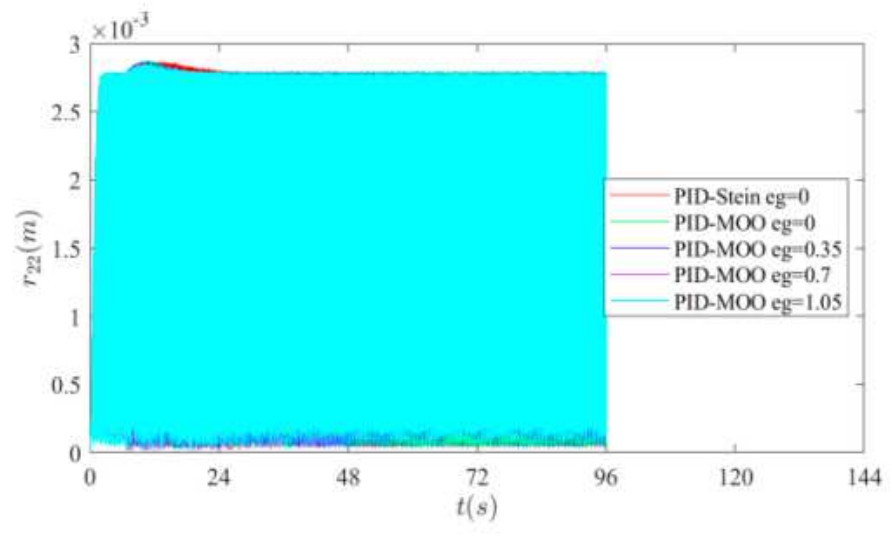

$$
m_{g}=-0.1
$$

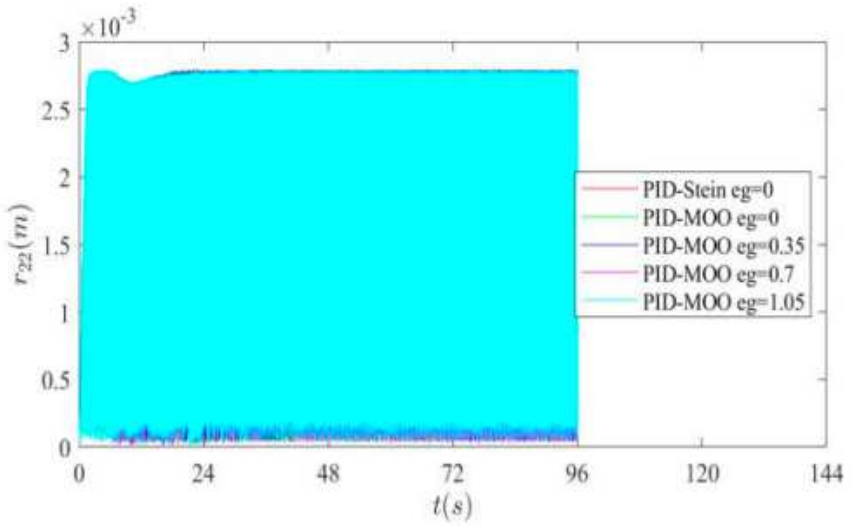

$m_{g}=0.1$

\section{Figure 10}

Robustness of optimized PID controller for to the load-self regulation coefficient

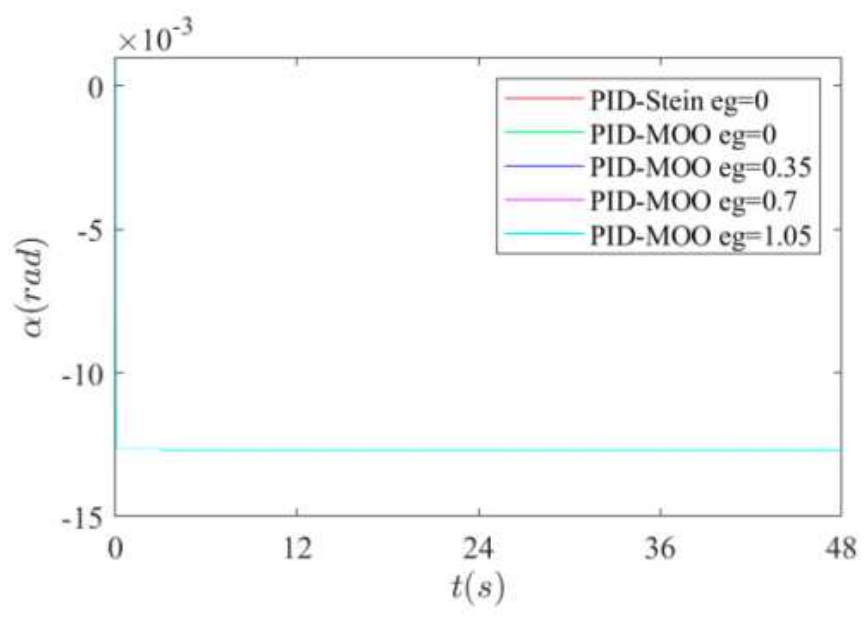

$m_{g}=-0.1$

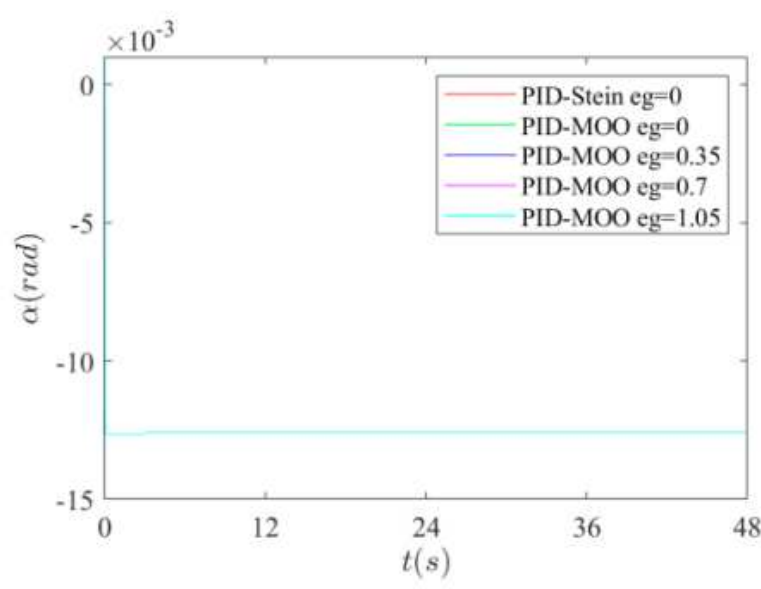

$m_{g}=0.1$

\section{Figure 11}

Robustness of optimized PID controller for torsion angle to the load-self regulation coefficient
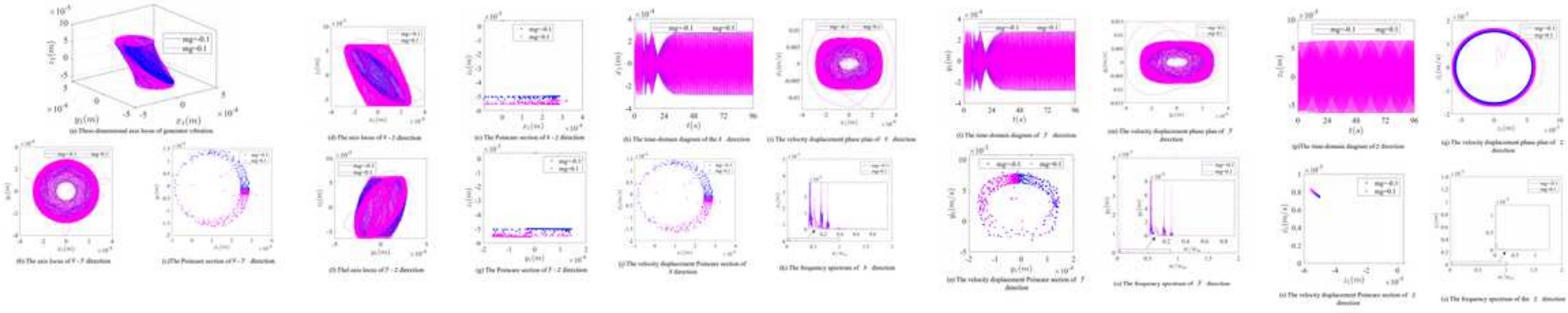

Figure 12 
The nonlinear dynamic characteristics of generator vibration
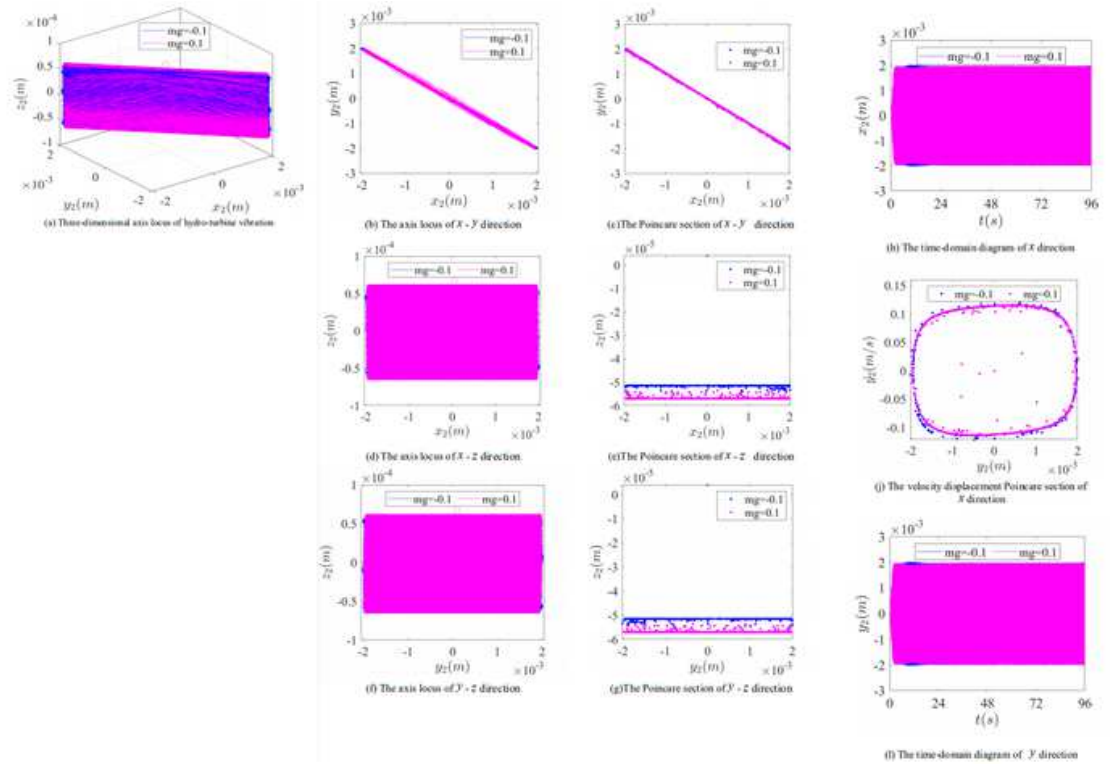
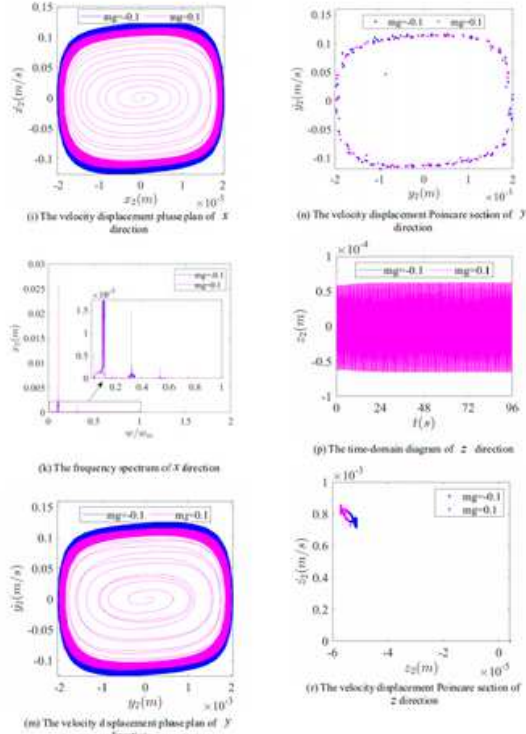
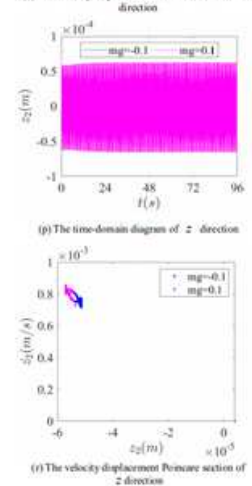

Figure 13

The nonlinear dynamic characteristics of hydro-turbine vibration 


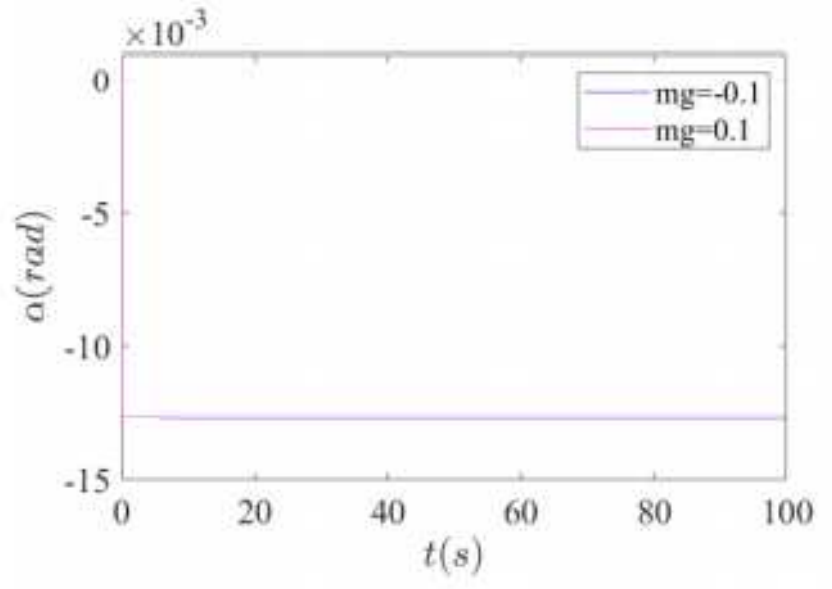

(a) Torsion vibration time-domain diagram

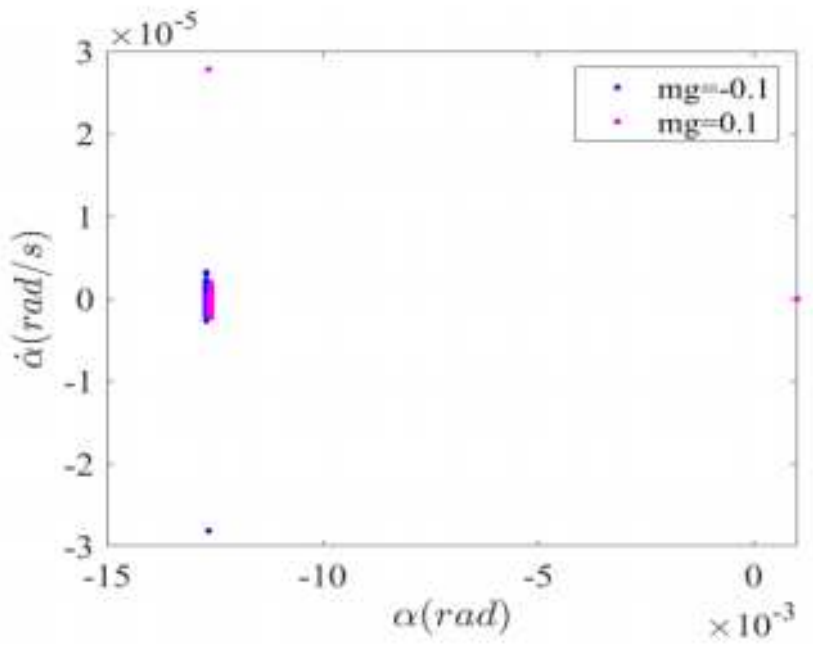

(c) Torsion vibration velocity displacement Poincare section

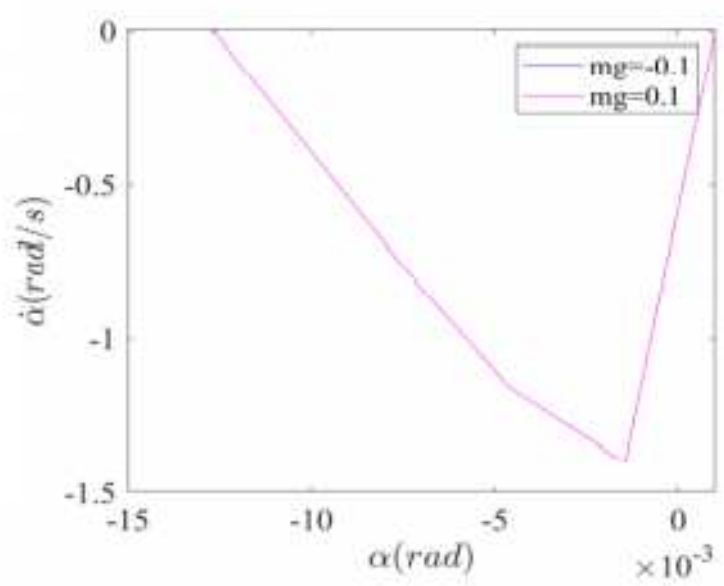

(b) Torsion vibration velocity displacement plase plan

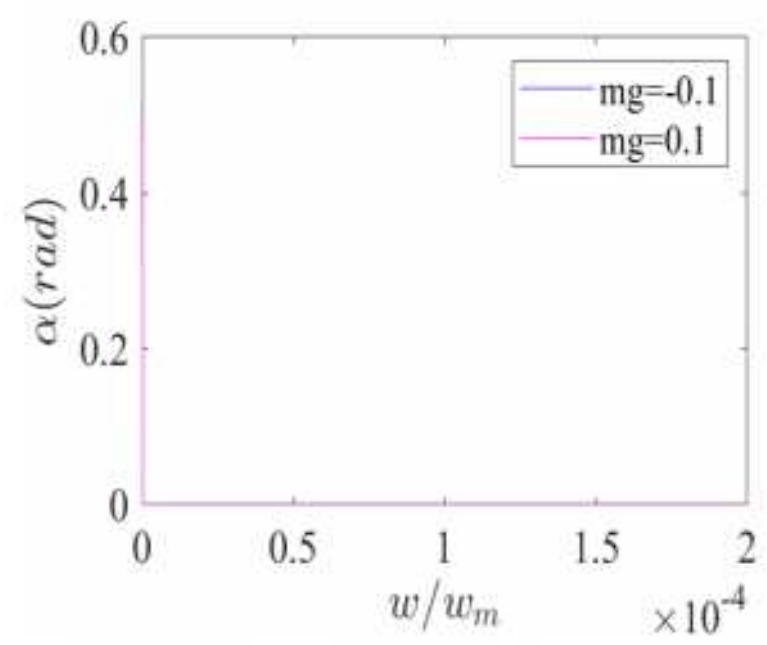

(d) Torsion vibration frequency spectrum

\section{Figure 14}

The nonlinear dynamic characteristics of torsion vibration 